\title{
Structural Analysis of the 50S Ribosomal Stalk
}

\author{
Dissertation \\ zur Erlangung des Doktorgrades \\ der Mathematisch-Naturwissenschaftlichen Fakultäten \\ der Georg-August-Universität zu Göttingen
}

vorgelegt von

Mihaela Ştefania Diaconu

aus Bukarest, Rumänien

Göttingen 2006 
D 7

Referent: Prof. Dr. Ralf Ficner

Korreferent: Prof. Dr. Oliver Einsle

Tag der mündlichen Prüfung: 05.07.2006 


\section{Table of contents}

Summary

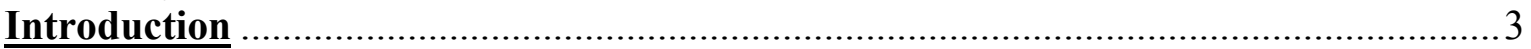

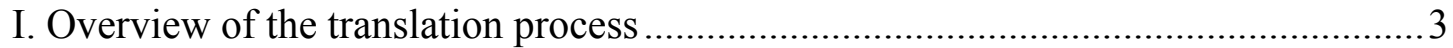

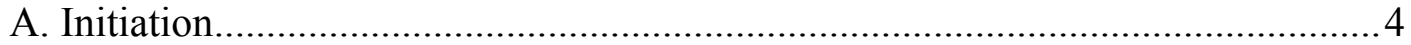

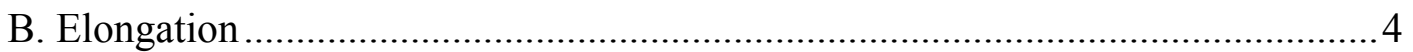

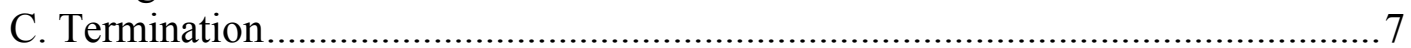

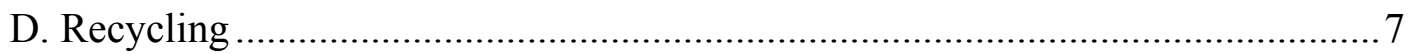

E. Action of antibiotics on the translation machinery ……………………............. 8

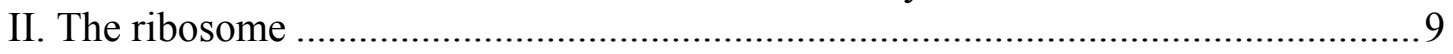

A. Components of the ribosome ……………………………..............................

B. Functional significance of the ribosomal elements............................................. 11

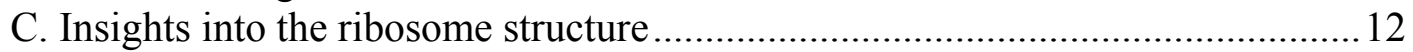

D. Modulators of the ribosomal activity................................................................. 14

1. Requirement of GTPase activity by EF-Tu and EF-G for translation.............. 14

2. GTP hydrolysis represents the driving force of translation.............................. 14

3. Ribosomal GTPases act as molecular switches ...............................................15

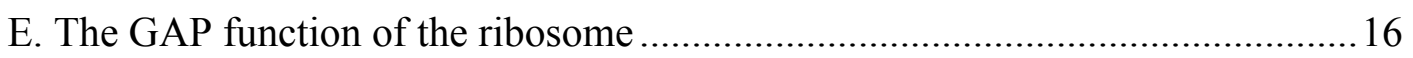

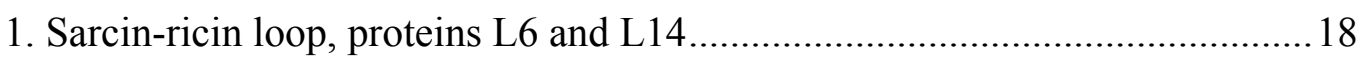

2. L11 protein and L10/L11 rRNA binding region ............................................ 19

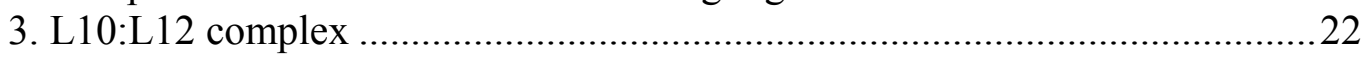

a. Characterization of the L10-L12 interaction ............................................22

b. Domain organization and dimerization mode of the L12 protein ................23

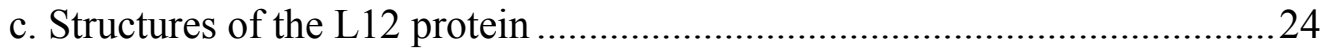

d. Different locations of the L12 protein on the ribosome ……………….......25

e. Phylogenetic comparison.........................................................................25

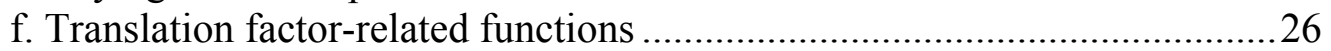

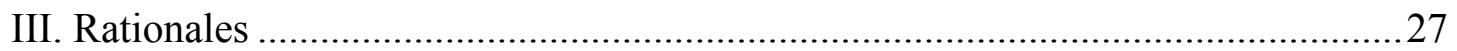

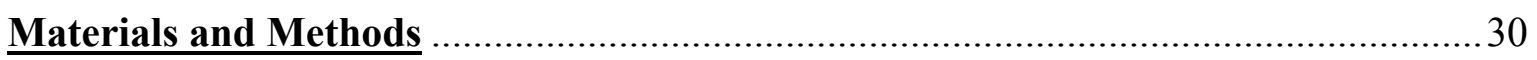

I. Molecular cloning ………………………………….................................... 30

A. Genomic DNA preparation.......................................................................

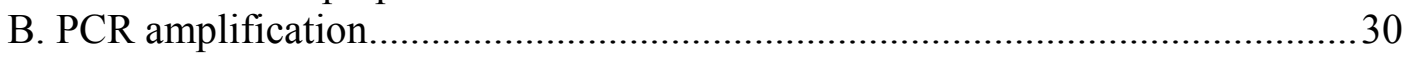

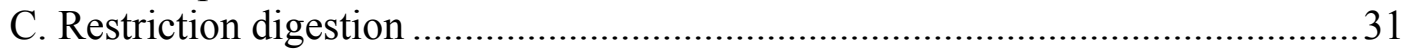

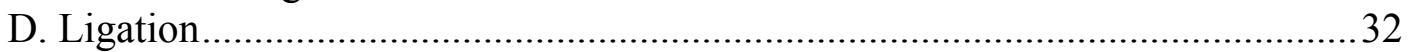

E. Competent cells preparation by calcium chloride treatment.................................32

F. Transformation of $E$. coli cells by heat shock .....................................................33

G. Mini-preparation of plasmid DNA ……………………...................................

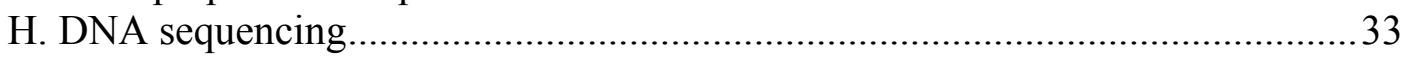

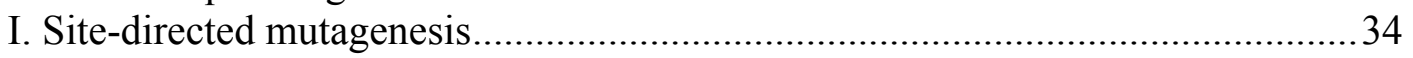

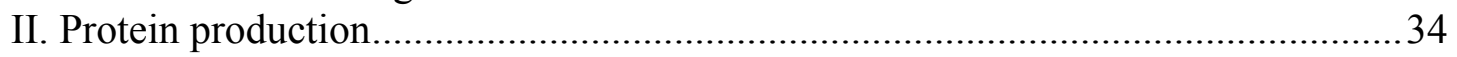

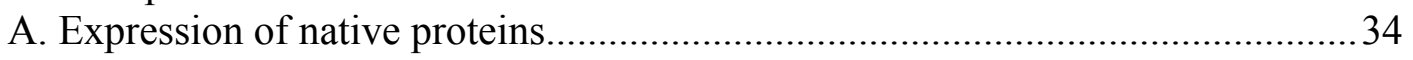

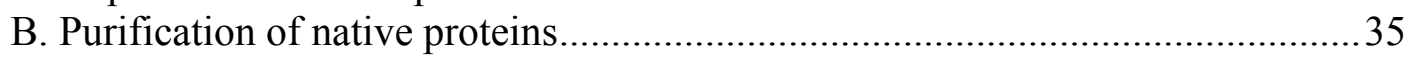

1. Purification of the aaeL10:L12 complex...................................................... 35

2. Purification of tmaL10:L12, tmaL10:L12 NTD, tmaL10:L12 NTD/hinge, tmaL10:ecoL12, tmaL10 ${ }_{\Delta 2 \mathrm{DBS}}$, tmaL12 CTD, tmaEF-Tu(Gd) complexes..........36

3. Purification of the ecoL10:L12 complex............................................................

4. Purification of the tmaL11 protein ................................................................... 37

5. Purification of TEV protease .......................................................................... 38 
C. Expression of the selenomethionine-derivatized protein..................................38

D. Purification of the selenomethionine-derivatized protein.................................39

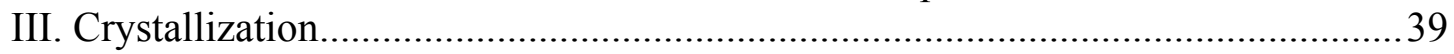

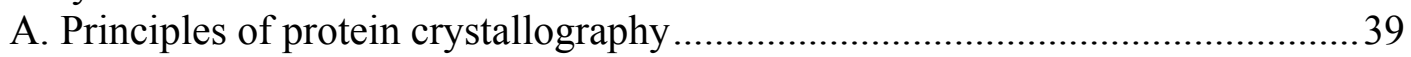

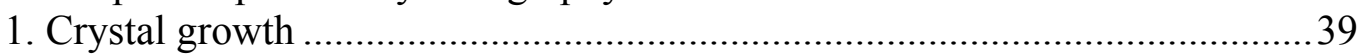

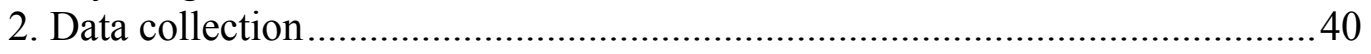

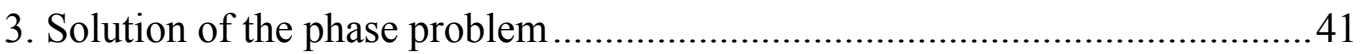

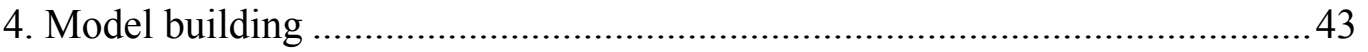

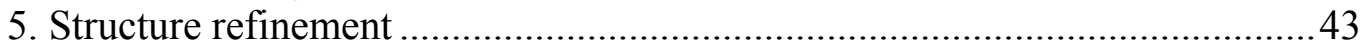

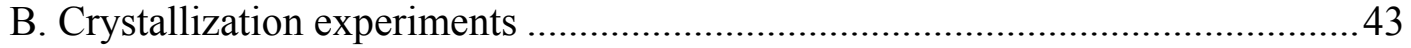

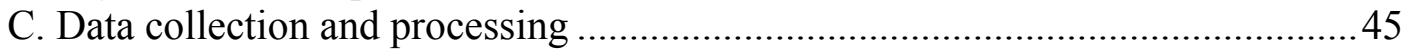

D. Phase generation, model building and refinement.............................................46

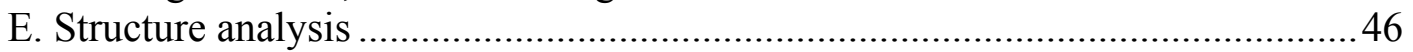

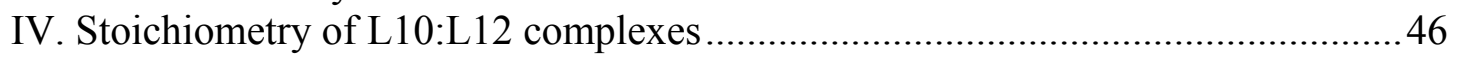

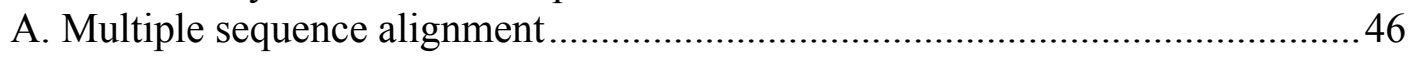

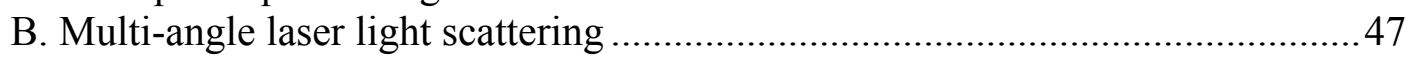

V. CD spectroscopy studies of tmaL10:L12 complex.............................................47

VI. Characterization of the interaction between $\operatorname{tmaL12}$ CTD and elongation factors

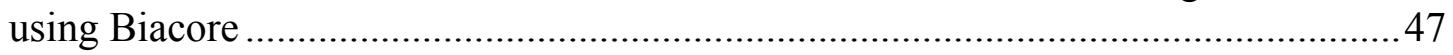

VII. Calorimetric analysis of the tmaL11:L12 CTD complex...................................48

VIII. Preparation of Thermotoga maritima ribosomes............................................... 48

IX. Measurement of the Thermotoga maritima ribosomal activity...........................49

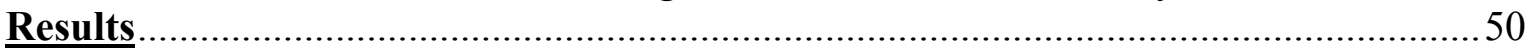

I. Expression screening of L10 and L12 proteins from different bacteria..................50

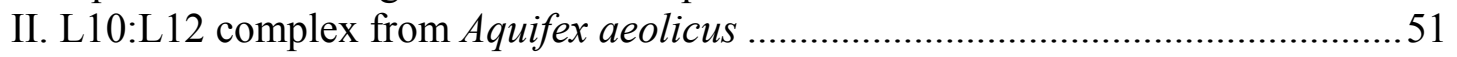

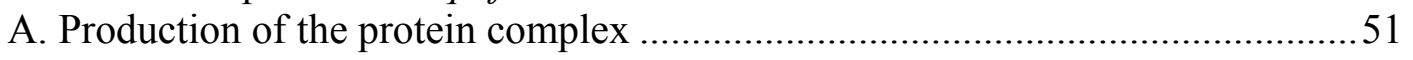

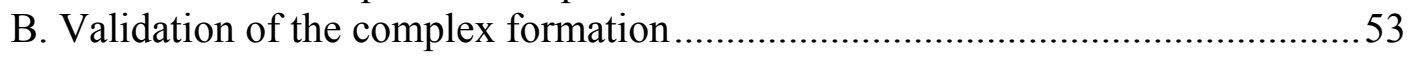

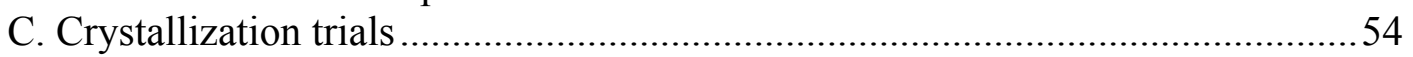

III. L10:L12 complex from Thermotoga maritima ..................................................5

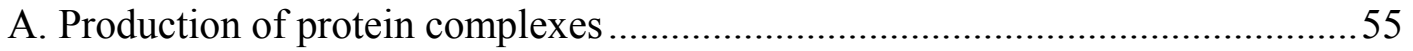

1. Native tmaL10:L12 and tmaL10:L12 NTD complexes ................................55

2. Selenomethionine-derivatized tmaL10:L12 NTD complex .........................57

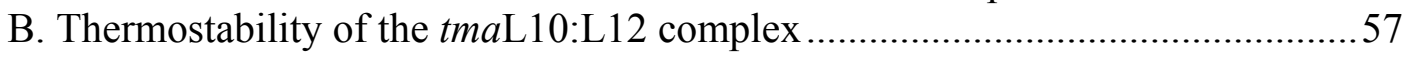

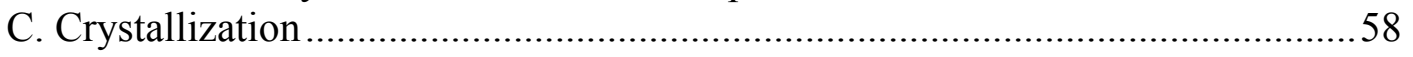

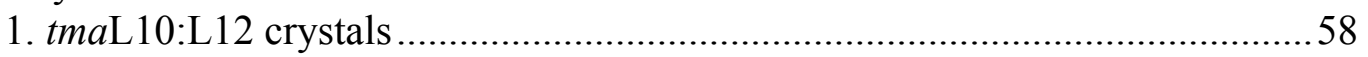

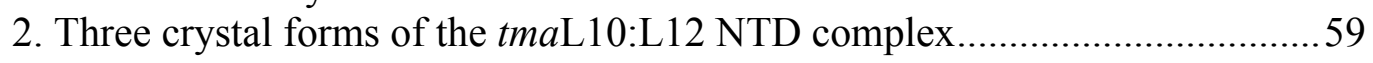

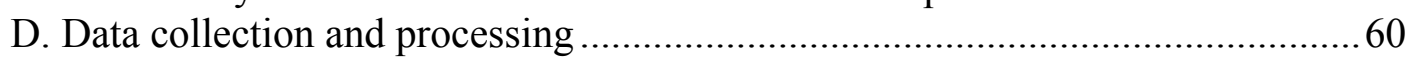

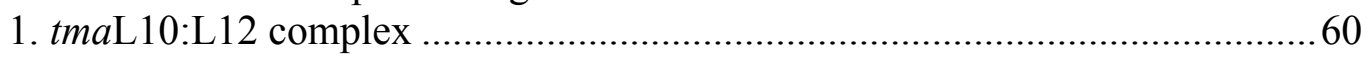

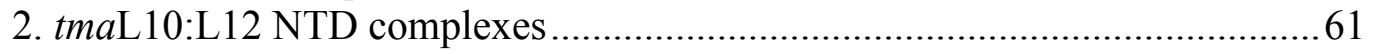

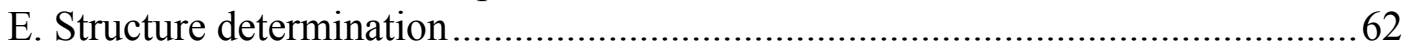

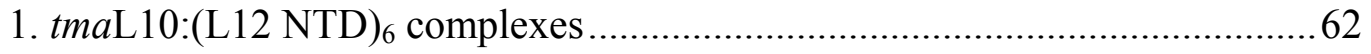

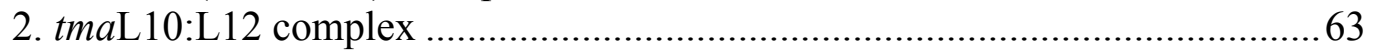

F. Refinement and quality of the model of the tmaL10:(L12 NTD) 6 crystal

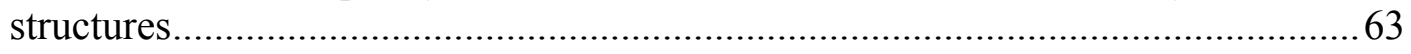

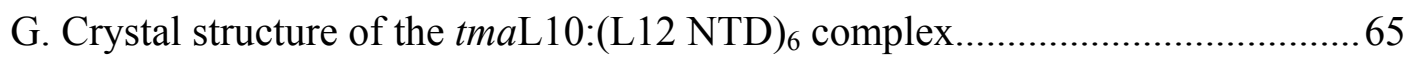

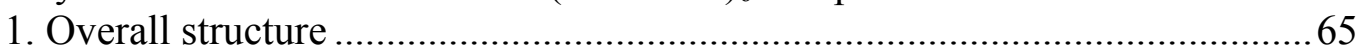

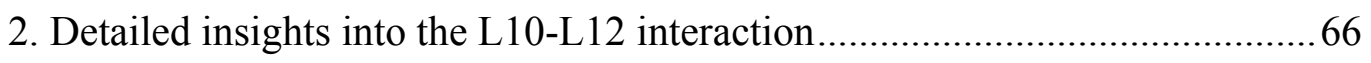

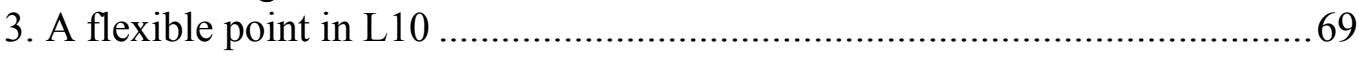

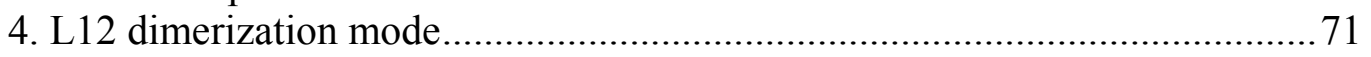




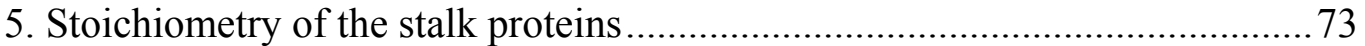

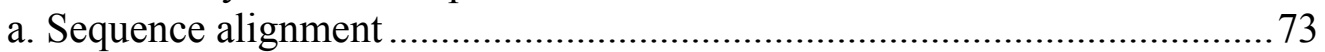

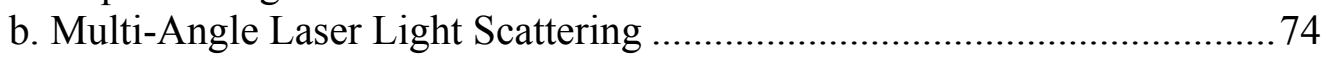

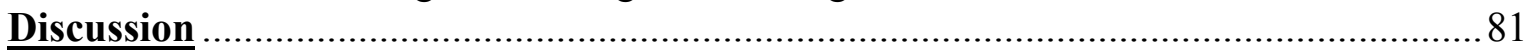

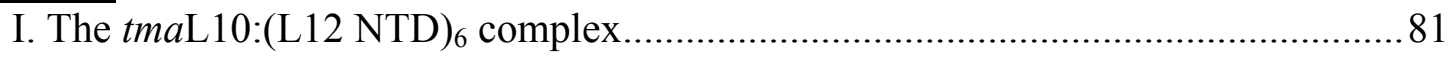

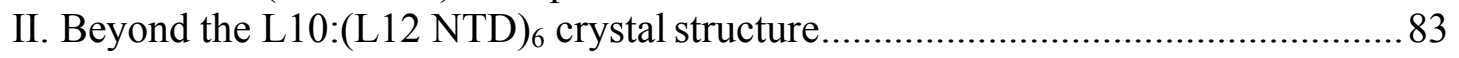

A. Placement of the tmaL10:(L12 NTD) ${ }_{6}$ structure on the 50 S ribosomal subunit 83

B. Cryo-EM reconstructions of L7/L12 stalk elements...................................... 84

C. Active sites of the L7/L12 stalk and their factor-related functions ....................84

III. The L7/L12 stalk: structural model and function in translation ..........................85

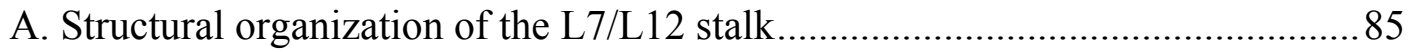

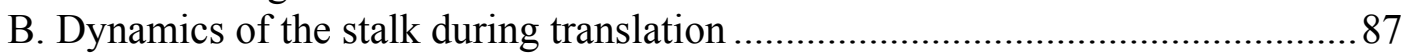

C. Mechanism of factor binding to the ribosome ............................................... 88

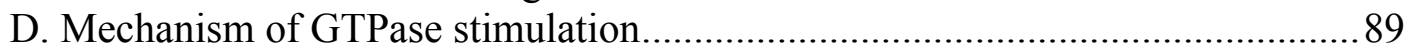

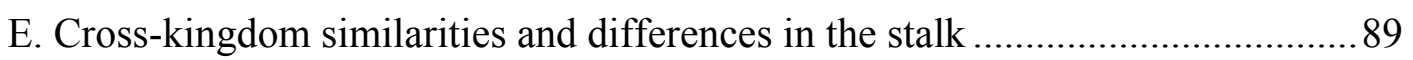

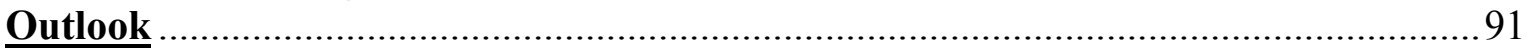

I. L10 ${ }_{\Delta \mathrm{DBS}}: \mathrm{L} 12$ complexes from Thermotoga maritima …......................................... 91

A. Production of the tmaL10 ${ }_{\triangle 2 \mathrm{DBS}}: \mathrm{L12}$ complex................................................. 91

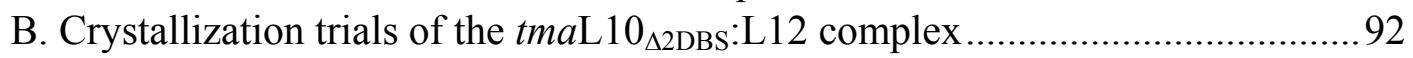

II. tmaL12 CTD and its interaction with elongation factors..................................92

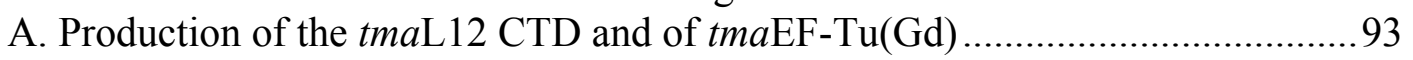

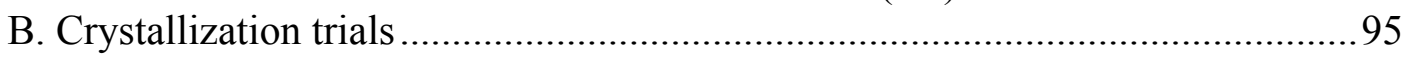

C. Interaction studies of the tmaL12 CTD and elongation factors by Biacore ........95

III. L11:L12 CTD complexes from Thermotoga maritima ......................................96

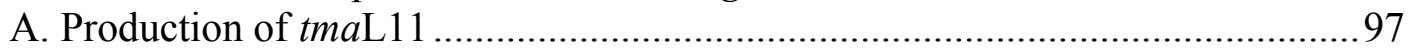

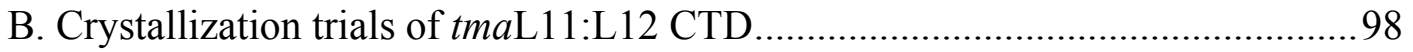

C. Interaction study of the tmaL11 and tmaL12 CTD by isothermal titration

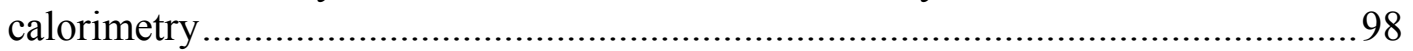

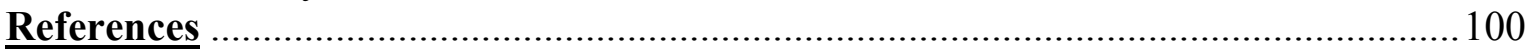

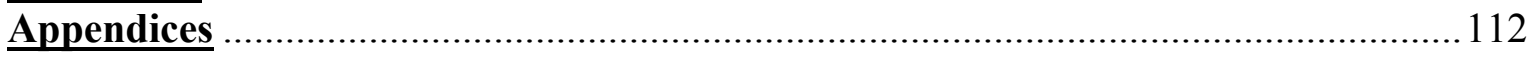

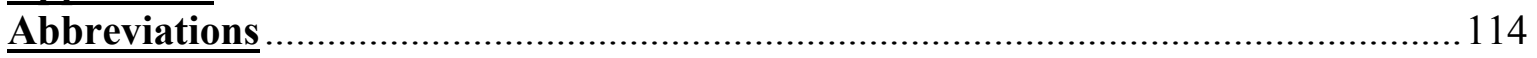

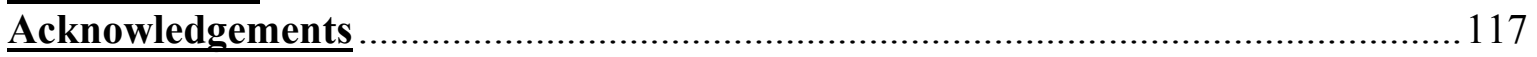

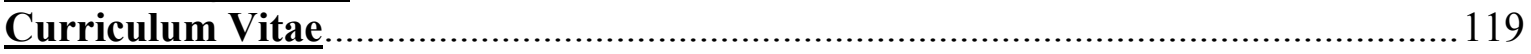




\section{$\underline{\text { Summary }}$}

Protein biosynthesis represents a dynamic process that takes place on the ribosome and is driven by translation factors. Some of these factors are GTP binding proteins. They possess a limited inherent GTPase activity that is stimulated by interactions with the ribosome in a region located on the large ribosomal subunit (GTPase associated region). This site comprises several 23S rRNA elements (L10/L11 rRNA binding region and sarcin-ricin loop) and r-proteins, such as L6, L11, L14, and the L7/L12 stalk. The latter corresponds to an extended feature of the 50S ribosomal subunit, encompassing multiple copies of protein L12 that are linked to the ribosomal RNA via L10. Numerous lines of evidence indicated that L12 is essential for both translation factor binding and stimulation of their GTPase activities. Functionally, L12 can be divided into an N-terminal domain (NTD) responsible for dimerization and interaction with L10, a C-terminal domain (CTD) necessary for factor-related functions, and an intervening flexible hinge.

Crystallographic studies of 50S subunits and 70S ribosomes hitherto failed to disclose the structure of the L7/L12 stalk, most probably due to the high mobility of the L12 hinge region. Thus, a complex anticipated to exhibit less flexibility was designed. It encompassed L10 and the NTD of L12 from the hyperthermophilic bacterium Thermotoga maritima. In the three crystal structures obtained, L10 displayed a globular NTD connected by a flexible loop to a long C-terminal $\alpha$-helix. The latter displayed different orientations relative to the L10 NTD in different crystal forms and harbored three consecutive binding sites for the L12 NTD dimers. Such a 1:6 (L10:L12) stoichiometry was unexpected, as a 1:4 ratio was well established in E. coli. The L12 NTDs formed dimers that fitted to a mode of dimerization reported for the protein in isolation, both in solution (Bocharov et al. 2004; Moens et al. 2005) and in crystalline environment (Wahl et al. 2000a). In the crystal structure of isolated T. maritima L12, the hinge region of one protomer exhibited an $\alpha$-helical shape, folded onto the L12 NTDs of the dimer, while in tmaL10:(L12 NTD) 6 , the hinge was found replaced by the C-terminal $\alpha$-helix of L10. Thus, it is likely that in complex with L10, the L12 hinges are flexible and unstructured, in agreement with several studies of this protein in solution.

In addition to obtaining the structure of tmaL10:(L12 NTD) 6 , attempts to solve the crystal structure of the full-length L10:L12 complex were also undertaken. While the 
crystallization of the complex from the hyperthermophilic bacterium Aquifex aeolicus proved to be unsuccessful, the corresponding complex from Thermotoga maritima yielded crystals that diffracted to $3.5 \AA$. The structure could be solved by molecular replacement using the tmaL10:(L12 NTD) 6 complex as a search model. No electron density could be detected for the L12 hinges and CTDs, consistent with a degradation of L12 during crystallization, as revealed by SDS-PAGE analysis of dissolved crystals. Comparisons of this structure to the three crystal structures obtained for tmaL10:(L12 NTD) 6 revealed a fourth orientation of the L10 C-terminal $\alpha$-helix-(L12 NTD) 6 element with respect to the L10 NTD, further supporting the notion of the presence of a flexible connection between these modules.

The in situ structure of an archaeal L10 NTD (a collaborative work with F. Schlünzen, J.M. Harms, Hamburg), enabled the positioning of the isolated tmaL10:(L12 NTD) 6 complex on the 50S ribosomal subunit. The L10 NTD was found to constitute a separate folding unit, necessary and sufficient to anchor the tmaL10:(L12 NTD) 6 complex on the L10/L11 rRNA binding region of the ribosome. The resulting model of a 50S subunit bearing a L10:(L12 NTD) 6 complex was confirmed by an excellent fitting into the cryoEM envelop of an E. coli 70S:EF-G:GDP:fusidic acid complex (N. Fischer, H. Stark, Göttingen). Based on these data and on structures of isolated L12, it was envisioned that the stalk is organized into three structural and functional elements, that are connected by flexible regions: (i) the stalk base, formed by the L10/L11 rRNA binding region, L11 and the L10 NTD, serving as attachment site for peripheral components; (ii) the C-terminal $\alpha$ helix of L10 in complex with L12 NTD dimers that constitute a movable platform carrying L12 hinges and CTDs; (iii) the highly mobile L12 CTDs attached to the mobile platform via the hinge regions. This arrangement was in agreement with L12 CTDs being active players in the dynamic functions of the stalk. Indeed, fast kinetic measurements using ribosomes with wild-type and mutant L12 (performed by U. Kothe, M.V. Rodnina, Witten; A.G. Tonevitski, Moscow) pinpointed L12 CTDs as initial interaction sites for translation factors, mediating their fast recruitment to the ribosome. These results also suggested that L12 CTDs activate GTP hydrolysis allosterically, a mechanism of action reminiscent of the regulators of G-protein signaling. Additionally, it can be hypothesized that L12 CTDs could either (i) remain bound to the factors' G-domains during their movement towards their ribosomal binding site or (ii) reach back to the ribosome-bound factors to stimulate their GTPase activities. 


\section{Introduction}

\section{Overview of the translation process}

The fundamental principles underlying protein biosynthesis are common throughout all forms of life. They involve the translation of the genetic information contained in messenger RNA into a protein sequence by a multimegadalton ribonucleoprotein particle, the ribosome. Surprisingly, an E. coli cell can host up to 20,000 of such organelles that, in total, consume more than $80 \%$ of the cellular energy during protein synthesis. Consequently, such a high energy need requires a tight regulation. One level of control is exerted by translation factors, which cycle on and off the ribosome as they perform their function at defined stages of translation (Table 1). A number of factors exhibit GTPbinding properties and can thereby be considered as molecular switches.

\begin{tabular}{lcc}
\hline \hline Translation phase & Prokaryotes & Eukaryotes \\
\hline \hline Initiation & IF1 & $\sim 12$ eIFs \\
& IF2 & \\
& IF3 & \\
\hline Elongation & EF-Tu & EF1 $\alpha$ \\
& EF-Ts & EF1 $\beta, \gamma, \delta$ \\
& EF-G & EF2 \\
& & EF3 (fungi) \\
\hline Termination & $\mathrm{RF} 1$ & $\mathrm{eRF} 1$ \\
& $\mathrm{RF} 2$ & - \\
& $\mathrm{RF} 3$ & $\mathrm{eRF} 3$ \\
\hline Ribosome recycling & $\mathrm{RRF}$ & - \\
\hline \hline
\end{tabular}

Table 1. Prokaryotic and eukaryotic protein factors involved in different translation phases. Factor GTPases are depicted in red.

Translation follows the basic model sketched many years ago by Watson (Watson 1964) and consists of the following phases: initiation, elongation, termination and recycling. 


\section{A. Initiation}

The first phase of protein synthesis in prokaryotes, the initiation (Figure 1), begins with the formation of a complex between the small ribosomal subunit (30S) and the initiation factor 3 (IF3). The latter mainly acts to prevent the association of the two ribosomal subunits (30S and 50S) (Gualerzi and Pon 1990). Additionally, IF3 monitors the correct binding of the mRNA and the initiator tRNA (a tRNA carrying formylmethionine or fMet-tRNA ${ }_{\mathrm{f}}^{\text {Met }}$ ) to the $30 \mathrm{~S}$ subunit (Hartz et al. 1989). The mRNA is anchored to the 30S subunit through a complementary base pairing between its Shine-Dalgarno sequence (ribosomal binding site) located upstream of the AUG start codon, and the 3'-terminal sequence of the 16S rRNA (anti Shine-Dalgarno sequence; ASD) (Shine and Dalgarno 1974). Consequently, the AUG start codon is positioned at the partial P site of the $30 \mathrm{~S}$ subunit. Next, IF1 joins the complex and is believed to indirectly guide the initiator tRNA to the $\mathrm{P}$ site (Carter et al. 2001). The resulting complex formed between the mRNA, 30S subunit, IF1 and IF3, interacts with the initiator tRNA, whose anticodon is complementary to the mRNA start codon (Gualerzi and Pon 1990). Subsequently, in the absence of GTP, the GTPase protein IF2 connects to the initiator tRNA on the 30S subunit (Weiel and Hershey 1982). Upon GTP binding, IF2 triggers a rapid 50S subunit association to the initiation complex and thereby reconstitutes the 70S ribosome. The IF2 G-domain contacts a region termed the "factor binding site" of the 50S subunit (Moreno et al. 1999; La Teana et al. 2001; Allen et al. 2005). IF1 and IF3 display a very low affinity for the 70S particle (Maitra et al. 1982) and rapidly dissociate from it, inducing a conformational rearrangement of the complex (Gualerzi and Pon 1990). Subunit association entails GTP hydrolysis by IF2, followed by its detachment from the 70S ribosome (Tomsic et al. 2000; Boelens and Gualerzi 2002). Curiously, IF2 dissociation is not a consequence of GTP hydrolysis (Tomsic et al. 2000).

\section{B. Elongation}

The resulting $70 \mathrm{~S}$ initiation complex enters the elongation phase (Figure 1). Elongation represents a cyclic process, which includes: the binding and selection of the aminoacyl-tRNA to be added, the peptidyl transferase reaction and the translocation. 


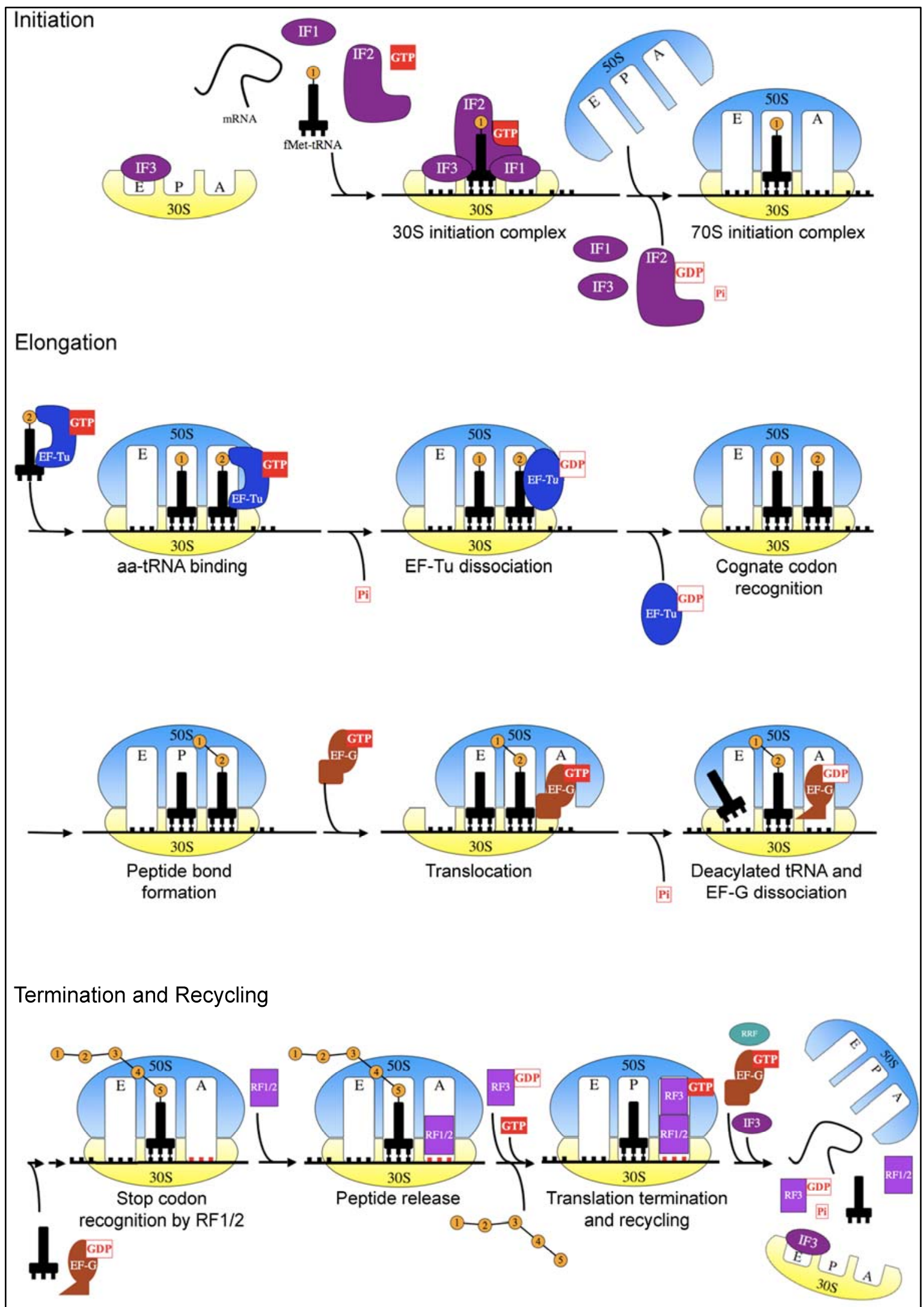

Figure 1. The prokaryotic translation. The genetic information encoded by the mRNA molecule is translated into a protein through a process comprising initiation, elongation of the peptide chain, termination and recycling of the translational apparatus elements (see main text for details). 
Firstly, a ternary complex EF-Tu:GTP:aminoacyl-tRNA presents the second aminoacyl-tRNA (aa-tRNA), whose anticodon must form complementary base pairs with the mRNA codon, at the A site (decoding site) of the ribosome. Following the initial aatRNA binding, non-cognate complexes are immediately rejected, while the near-cognate (codon-anticodon complexes with one base pair mismatch) and cognate are recognized (Rodnina et al. 2002). Codon recognition has two important consequences: the first is the stabilization of the codon-anticodon interaction and the second, the stimulation of the GTP hydrolysis by EF-Tu. Upon the latter, EF-Tu undergoes extensive structural changes (Berchtold et al. 1993; Kjeldgaard et al. 1993; Polekhina et al. 1996). The resulting EFTu:GDP exhibits a very low affinity for the aminoacyl-tRNA and dissociates from the ribosome (Dell et al. 1990). The recycling of inactive EF-Tu:GDP complex to the active GTP complex is performed by EF-Ts, a guanine-nucleotide exchange factor (GEF) (LucasLenard and Lipmann 1966). Interestingly, during the initial selection step, a near-cognate duplex is not rejected before GTP hydrolysis. However, it will be edited during a proofreading step, prior to the peptide bond formation (Rodnina et al. 2002).

Next, the peptidyl transferase reaction, characterized by the formation of a peptide bond between the peptidyl-tRNA from the P site and the aminoacyl-tRNA accommodated in the A site, occurs. The peptide chain is transferred to the A site aa-tRNA, leaving a deacylated tRNA in the P site. This reaction takes place on the 50S subunit (in the peptidyl transferase center, PTC) (Kaziro 1978) and is catalyzed by 23S rRNA elements (Noller et al. 1992; Nissen et al. 2000a). Recently, it has been hypothesized that, rather than catalyzing the reaction, rRNAs function as entropy traps, bringing reactants close enough to each other to allow the transpeptidase reaction (Sievers et al. 2004).

The last step of the elongation process, the translocation, results in the synchronous movement of the two tRNAs and mRNA by one codon (Wilson and Noller 1998). Precisely, the deacylated tRNA moves from the P to the E site and the peptidyl-tRNA, from the A to the $\mathrm{P}$ site, thus leaving the A site vacant for a new round of elongation. The process of translocation is catalyzed by the EF-G (Rodnina et al. 1997). Three models for the translocation mechanism have been proposed. In a first model, the translocation would occur before GTP hydrolysis (Inoue-Yokosawa et al. 1974), whereas a second suggests that the translocation would take place after the GTP is hydrolyzed (Rodnina et al. 1997). In a third model, the translocation would be initiated prior to GTP hydrolysis and completed afterwards (Zavialov et al. 2005). Concomitantly with the translocation process, 
EF-G suffers extensive conformational changes, thereby inducing the tRNA:mRNA complex displacement. Using cryo-electron microscopy, it was established that, both EF-G binding and the subsequent GTP hydrolysis, lead to a ratchet-like movement of the $30 \mathrm{~S}$ subunit relative to its 50S counterpart (Frank and Agrawal 2000). Upon tRNA-mRNA coordinated movement, the ribosome returns to its initial state. Finally, EF-G:GDP and Esite-deacylated tRNA dissociate from the ribosome. Interestingly, crystal structure comparisons of EF-G:GDP (Ævarsson et al. 1994; Czworkowski et al. 1994) with the EFTu:GTP:tRNA ternary complex (Nissen et al. 1995), as well as cryo-EM analysis (Stark et al. 1997b; Agrawal et al. 1998), revealed that both complexes adopt a similar shape when interacting with the ribosome, suggesting a molecular mimicry (reviewed in (Nyborg 1998; Kristensen et al. 2002)).

\section{Termination}

The elongation cycle is repeated (with a rate of approximately 12 amino acids per second in bacteria (Gualerzi and Pon 1990)) until a termination codon appears in the A site (Figure 1, in red). The stop codon is then identified by a class-1 release factor (RF1 or RF2). RF1 recognizes UAA and UAG, whereas RF2 is specific for UAA and UGA (Kisselev and Buckingham 2000). Upon codon recognition, the peptide chain is hydrolyzed and released from the $\mathrm{P}$ site-tRNA. Cryo-electron microscopy results demonstrated that RF1/2 binds to the termination codon and, at the same time, contacts the peptidyl transferase center (Rawat et al. 2003; Rawat et al. 2006). However, it is not clear whether they are directly involved in the peptide chain release or indirectly induce this reaction by signaling the ribosome. Next, the class-2 release factor (RF3), a factor GTPase, induces the detachment of the class-1 RFs from the ribosome. Specifically, RF3 in a GDPbound state contacts class-1 RFs, a GDP to GTP exchange occurs which subsequently triggers the RF1/2 release (Zavialov et al. 2001). Finally, the hydrolysis of RF3:GTP elicits its dissociation from the ribosome (Zavialov et al. 2001).

\section{Recycling}

Following the release of the peptide chain, the ribosome, carrying the deacylated tRNA in the $\mathrm{P}$ site, and the mRNA are disassembled by a complex composed of the ribosome recycling factor (RRF), EF-G and GTP, through a GTP hydrolysis-dependent 
process (Karimi et al. 1999). IF3 then binds to the 30S subunit and induces the release of the deacylated tRNA (Figure 1). The dissociated ribosomal subunits can now reenter a new round of protein synthesis.

\section{E. Action of antibiotics on the translation machinery}

The translation machinery, especially the peptidyl transferase center and the decoding site, represent the target of numerous classes of antibiotics (briefly outlined in Table 2; reviewed in (Wilson et al. 2005)). Several antibiotics (e.g. thiostrepton, kirromycin) bind at defined locations on the ribosome, inhibit a specific conformation, and thereby impair further protein synthesis. An antibiotic that directly interacts with a translation factor is fusidic acid. It prevents EF-G dissociation from the ribosome upon GTP hydrolysis, thus blocking further rounds of elongation. The above mentioned properties of antibiotics were extensively exploited in cryo-electron microscopy studies (Stark et al. 1997b; Agrawal et al. 1999; Stark et al. 2000; Stark et al. 2002; Valle et al. 2003a). In this way, different phases of translation could be analyzed by stalling the ribosome in a specific conformation.

\begin{tabular}{ll}
\hline \hline Antibiotic & Effect \\
\hline Tetracyclin & Inhibits aminoacyl-tRNA A-site binding \\
Streptomycin & Induces misreading \\
Kirromycin & Blocks EF-Tu after GTP hydrolysis \\
Chloramphenicol & Inhibits peptidyl transferase \\
Thiostrepton & Inhibits translocation \\
Fusidic acid & Induces arrest in the posttranslocational phase \\
Erythromycin & Inhibits peptide elongation \\
Puromycin & Inhibits peptide release \\
\hline \hline
\end{tabular}

Table 2. Antibiotic action on prokaryotic translation.

A number of antibiotics inhibit bacterial translation with sufficient selectivity to be suitable for antibacterial therapy. Hence, it is clear that a detailed structural knowledge of the protein synthesis mechanism represents a valuable source of information in biomedical research, providing tools for the design of new drugs aiming at impairing the proliferation of resistant pathogens. 


\section{The ribosome}

A further understanding of the processes underlying protein synthesis is correlated with a thorough characterization of the molecular mechanisms by which ribosomes exert their function. Remarkable contributions in deciphering the ribosome and its ligands were made in the last decade by two major advances, namely cryo-electron microscopy and X-ray crystallography.

\section{A. Components of the ribosome}

Ribosomes were first identified by Palade as "small particulate components of the cytoplasm" (Palade 1955). Next, they were characterized as particles detected by ultracentrifugation of cell lysates and designated according to their rates of sedimentation: $70 \mathrm{~S}$ for bacterial ribosomes and 80S for ribosomes of eukaryotic cells (Taylor et al. 1967). All ribosomes consist of two subunits of unequal size: the small (30S in bacteria and $40 \mathrm{~S}$ in eukaryotes) and the large (50S and 60S, respectively) subunits. Both encompass several ribosomal RNAs (rRNAs) and numerous proteins (r-proteins), the latter being named S or $\mathrm{L}$, depending on their location on the small or large subunit, respectively (Table 3).

\begin{tabular}{|c|c|c|c|}
\hline \multicolumn{4}{|c|}{ Prokaryotes (E. coli) } \\
\hline Characteristics & Ribosome & Small Subunit & Large Subunit \\
\hline Size & $70 \mathrm{~S}(2.5 \mathrm{MDa})$ & $30 \mathrm{~S}(0.9 \mathrm{MDa})$ & $50 \mathrm{~S}(1.6 \mathrm{MDa})$ \\
\hline rRNAs & & 16S rRNA (1542 Nt) & $\begin{array}{l}\text { 23S rRNA }(2904 \mathrm{Nt}) \\
5 \mathrm{~S} \text { rRNA }(120 \mathrm{Nt})\end{array}$ \\
\hline Proteins & & 21 Proteins & 36 Proteins \\
\hline \multicolumn{4}{|c|}{ Eukaryotes (Mammals) } \\
\hline Characteristics & Ribosome & Small Subunit & Large Subunit \\
\hline Size & $80 \mathrm{~S}(4.2 \mathrm{MDa})$ & $40 \mathrm{~S}(1.4 \mathrm{MDa})$ & $60 \mathrm{~S}(2.8 \mathrm{MDa})$ \\
\hline rRNAs & & 18S rRNA (1874 Nt) & $\begin{array}{l}\text { 28S rRNA }(4718 \mathrm{Nt}) \\
5.8 \mathrm{~S} \text { rRNA }(160 \mathrm{Nt}) \\
5 \mathrm{~S} \text { rRNA }(120 \mathrm{Nt})\end{array}$ \\
\hline Proteins & & 33 Proteins & 49 Proteins \\
\hline
\end{tabular}

Table 3. Composition of the prokaryotic and eukaryotic ribosomes.

Eukaryotic ribosomes are larger and present an increased protein content as compared to their prokaryotic counterparts. The primary sequences of both rRNA and r- 
proteins of E. coli ribosomes were completely elucidated more than two decades ago (Brosius et al. 1980).

The 30S exhibits the following landmarks: the head, representing one third of volume, connected by a neck to the rest of the components, the shoulder, the platform, and the body with a spur (toe). The 50S subunit has a "crown" appearance: a hemispherical body with three protrusions, namely the L1 protuberance, the central protuberance (displaying 5S rRNA) and the L7/L12 stalk (Figure 2). The subunits are associated via multiple bridges, mostly between rRNA elements (Yusupov et al. 2001). Between them lies an internal cavity, containing three binding sites for tRNAs, designated the A (acceptor site of the Aminoacylated tRNA), P (Peptydil-tRNA site), and E (Exit for deacylated tRNA) sites, respectively.

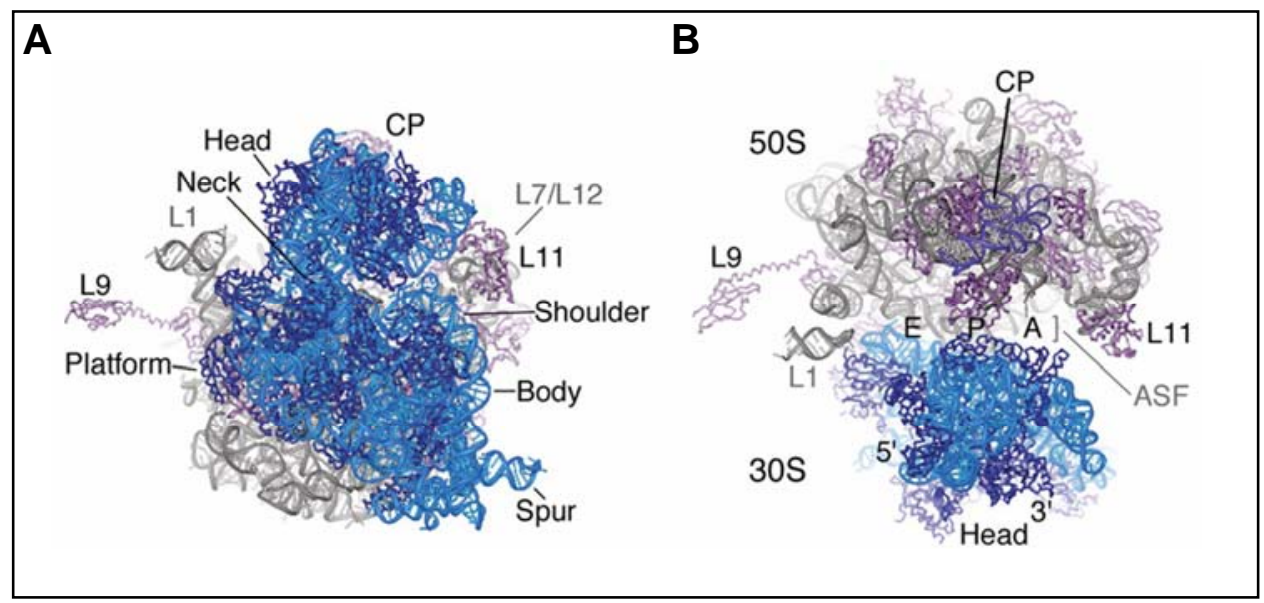

Figure 2. Structure of the intact E. coli 70 S ribosome. (A) View from the solvent side of the $30 \mathrm{~S}$ subunit. rRNA and proteins in the 30S subunit are colored in light blue and dark blue, respectively. 23S rRNA and proteins in the 50S subunit are in gray and magenta, respectively. 30S features include head, neck, platform, body, shoulder, and spur. 50S features encompass L1 (protein L1/rRNA arm), CP (central protuberance), ASF (A-site finger, labeled in (B)), and L11 (protein L11/rRNA arm). The approximate location of proteins L7/L12 and the tip of the ASF, not observed in the structure, are labeled in gray. (B) View rotated $90^{\circ}$ on the horizontal axis compared to (A). Letters indicate the approximate location of the aminoacyl (A), peptidyl (P), and exit (E) tRNA binding sites at the subunit interface. The 5' to $3^{\prime}$ direction of mRNA, which threads around the neck region of the 30S subunit, is also indicated (reproduced from Schuwirth et al., 2005; PDB accession codes: 2AVY, 2AW4).

The bacterial rRNA, accounting for two thirds of the ribosomal mass, comprises one rRNA form (16S rRNA) in the small subunit and two types of rRNA (23S rRNA and 5S rRNA, respectively) in the large subunit (Wittmann et al. 1982). As the tRNA, it has been established that the rRNA forms characteristic secondary structures (organized in four domains in $16 \mathrm{~S}$ rRNA and six domains in $23 \mathrm{~S}$ rRNA) by complementary base pairing 
(Gutell 1996), which further fold into distinct three-dimensional structures (Ban et al. 2000; Wimberly et al. 2000).

Ribosomal proteins are present in one copy each per ribosome, with the exception of L7/L12, displaying four copies in E. coli (Subramanian 1975). Generally, the net charge of the ribosomal proteins is basic, in order to neutralize the negative rRNA backbone (Klein et al. 2004). The proteins appear unevenly distributed within the ribosome: the inter-subunit sides are poor in proteins (only the S12 protein is found at the decoding center) (Brodersen et al. 2002), whereas at the mRNA entry site (Yusupova et al. 2001), the factor binding site (Ban et al. 1999) and the exit of the polypeptide tunnel (Klein et al. 2004), proteins are present in higher number and some have been identified as active players in these processes.

\section{B. Functional significance of the ribosomal elements}

Ribonucleoprotein particles, later recognized as ribosomes, were shown to participate in protein synthesis approximately fifty years ago (Littlefield et al. 1955). Subsequently, the reconstitution of the small and large subunits in vitro using their intrinsic components, yielded functionally active ribosomes in protein synthesis (Traub and Nomura 1968; Nierhaus and Dohme 1974). Despite these discoveries, the attribution of specific functions to certain ribosomal components has been a matter of debate: at first, ribosomal proteins were seen as key players in protein synthesis, whereas rRNAs were thought to exert a scaffolding role. However, in 1992, Noller and coworkers clearly demonstrated that the rRNA from the large ribosomal subunit (stripped of almost all of its proteins) was able to catalyze peptide bonds (Noller et al. 1992). This discovery, produced a shift of paradigm that supported the implication of rRNAs in several ribosomal functions, including potential catalytic properties at the peptidyl transferase center ("the ribosome is a ribozyme": (Nissen et al. 2000a)), an interaction with mRNA during the initiation and translocation of protein synthesis, or its predominance at the decoding site (discussed by (Steitz and Moore 2003)). Notably, the corresponding rRNA elements were found highly conserved throughout kingdoms.

The current view reconciles both above mentioned notions: the rRNA is prevalently detected at active sites, but is assisted, both structurally and functionally, by r-proteins. Most probably evolved to replace an inefficient rRNA, numerous ribosomal proteins 
synergically cooperate with rRNAs in order to promote the translation (Draper and Reynaldo 1999; Wilson and Nierhaus 2005). Consistently, several ribosomal proteins were found essential in some translational processes, i.e. S12 in decoding, L11 and L7/L12 in the interaction with translation factors. Thus, it can be concluded that in order to fulfill crucial functions, such as mRNA decoding, peptide bond formation, tRNA and mRNA translocation, or folding of the nascent polypeptide, a dynamic interplay occurs between the different ribosomal constituents. Moreover, in response to substrate binding (tRNAs, mRNA) and factor interaction, the ribosome changes its structure and takes an active part in all the steps of translation.

\section{Insights into the ribosome structure}

By enabling the visualization of the ribosome at atomic resolution, X-ray crystallography proved to be extremely valuable for the interpretation of different steps underlying protein synthesis (discussed in (Al-Karadaghi et al. 2000; Nissen et al. 2000b; Ramakrishnan 2002; Nilsson and Nissen 2005; Ogle and Ramakrishnan 2005)). This project was promoted in 1980, with the first attempts to crystallize Bacillus stearothermophilus ribosomes (Yonath et al. 1980), and significantly improved within the last decade.

Recently, crystal structures of isolated ribosomal subunits revealed new insights into the organization of the translational machinery. Several structures of the $30 \mathrm{~S}$ subunit from a thermophilic bacterium were determined at $5.5 \AA$ (Clemons et al. 1999), $4.5 \AA$ (Tocilj et al. 1999), $3.3 \AA$ (Schluenzen et al. 2000) and $3.05 \AA$ (Wimberly et al. 2000) resolution. In recent crystal structures of the small ribosomal subunit, all the ordered rRNAs (accounting for $99 \%$ of the $16 \mathrm{~S}$ rRNA) were traced in the electron density, along with 20 associated proteins.

Of equal importance were the structures of the $50 \mathrm{~S}$ subunit, obtained from a mesophilic bacterium at $3.1 \AA$ resolution (Harms et al. 2001) and from an archaeon at $5 \AA$ (Ban et al. 1999). A major breakthrough was the $2.4 \AA$ resolution of the large ribosomal subunit, the first high-resolution structure of a ribosomal subunit (Ban et al. 2000). It comprises most of the 50S subunit, including a detailed structure of the peptidyl transferase center and several components of the translation factor binding site. 
Furthermore, it strongly supports the notion that rRNA is responsible for the peptide bond formation, since the closest protein to the peptidyl transferase center is $18 \AA$ away.

In addition, high-resolution structures of ribosomal subunits in complex with numerous ligands and translation factors (Nissen et al. 2000a; Schmeing et al. 2002) or antibiotics (Brodersen et al. 2000; Carter et al. 2000; Pioletti et al. 2001; Schlunzen et al. 2001) were determined. Complexes of ribosomes with translation factors revealed some of the conformational rearrangements that the ribosome undergoes in response to their interaction. The 30S:IF1 complex revealed the location of IF1 at the A site of the $30 \mathrm{~S}$ subunit (Carter et al. 2001). Studies performed by Ogle and coworkers on the 30S subunit in complex with mRNA and tRNA showed that the decoding center is built by parts of helices 18, 34, 44 of $16 \mathrm{~S}$ rRNA. Importantly, residues responsible for monitoring the quality of codon-anticodon interactions were pinpointed. Thus, helix 44, through its A1493 and $\mathrm{A} 1492$, contacts the $1^{\text {st }}$ and the $2^{\text {nd }}$ position of the codon-anticodon duplex, whereas the $3^{\text {rd }}$ position is monitored less stringently by a contact from G530 (Ogle et al. 2001).

In parallel, the crystal structure of the 70S ribosome in complex with mRNA and tRNA from Thermus thermophilus, first at $7.8 \AA$ (Cate et al. 1999), and subsequently at $5.5 \AA$ resolution (Yusupov et al. 2001) was solved. Separate ribosomal proteins and rRNA components, for which high-resolution structures had been obtained in early X-Ray crystallographic and NMR studies, could then be recognized at these resolutions and modeled into the electron density. The resulting models revealed the relative orientations of tRNAs within the ribosome, providing new insights into the decoding phase. They also unraveled the path of the mRNA throughout the $30 \mathrm{~S}$ subunit and the bridges formed between the two subunits. Recently, two structures of the 70S ribosome from Escherichia coli at $9 \AA$ (Vila-Sanjurjo et al. 2003) and at $3.5 \AA$ resolution (Schuwirth et al. 2005) were reported. They provided a detailed view of the interface between the small and large ribosomal subunits and the conformation of the peptidyl transferase center in the context of the intact ribosome. They also suggested a model for the final movements of mRNA and tRNAs during translocation. E. coli structures are of great importance, as most of the genetic, biochemical and biophysical data are available from this organism.

Taken together, these structural studies highlighted the roles of individual ribosomal subunits in translation. The small ribosomal subunit plays a crucial role in decoding and validating the accuracy of the codon-anticodon base pairing. The large subunit participates to the peptide bond formation. Additionally, it contains a region that 
was identified as a common translation factor GTPase binding domain. While both processes underlying decoding and reaction at the peptidyl transferase center were clarified by several approaches, including crystallography, the mechanisms of factor GTPases binding to the ribosome are still not entirely understood.

\section{Modulators of the ribosomal activity}

\section{Requirement of GTPase activity by EF-Tu and EF-G for translation}

As described previously, the ribosome orchestrates protein synthesis, playing crucial roles in: (i) decoding, (ii) peptide bond formation, and (iii) translocation of the tRNAs and mRNA. Remarkably, it was shown that the ribosome is able to fulfill these functions by itself (Gavrilova et al. 1976). Indeed, with an appropriate mRNA matrix and aminoacylated-tRNAs, the ribosome is capable to synthesize peptide chains in the absence of elongation factors and GTP, performing a residual translation. Moreover, EF-Gindependent translocation occurs spontaneously in vitro, suggesting that this phenomenon resides entirely within the structure of the ribosome (Spirin 1985). However, it was also established that the elongation factor-free translation is inaccurate and does not meet the cell requirements. Therefore, in order to exert its function at physiological rates, the ribosome needs to associate with elongation factors (EF-Tu and EF-G), that act as catalysers improving its intrinsic properties.

\section{GTP hydrolysis represents the driving force of translation}

The high speed and fidelity of protein synthesis in vivo is achieved at the expense of energy consumption. This energy is provided by the hydrolysis of GTP coupled to EFTu and EF-G. Similarly, GTPase activity is also required for the function of IF2 and RF3 (Bourne et al. 1991).

The role of GTP hydrolysis by IF2, known to bind to the initiator tRNA on the 30S ribosomal subunit and to trigger subunit association, remains unclear. In contrast, GTPase activity by EF-Tu is better characterized and is coupled to the A-site aa-tRNA binding and correct codon-anticodon interaction (Pape et al. 1998). Through conformational changes, GTP hydrolysis elicits the dissociation of EF-Tu from the ribosome. The mechanism of GTP hydrolysis by EF-G is not clearly elucidated. Kinetic studies suggested that EF-G is a motor protein that, upon rapid GTPase reaction, drives the translocation (Rodnina et al. 
1997). A different model suggested that GTP hydrolysis by EF-G is not required for translocation, but for the release of EF-G (Inoue-Yokosawa et al. 1974); a similar observation was made for RF3, responsible for detaching the decoding release factors 1 and 2 from the ribosome (Zavialov et al. 2001).

\section{Ribosomal GTPases act as molecular switches}

Guanine Nucleotide-Binding Proteins (GNBPs) cycle between the GTP- and GDPbound states (Vetter and Wittinghofer 2001) (Figure 3). The transition from the GDP to the GTP-bound forms was shown to occur spontaneously in vitro. However, in vivo, this reaction is accelerated by Guanine nucleotide-Exchange Factors (GEFs). The GEF forms a complex with the GDP-bound protein, causing the dissociation of GDP. The resulting complex can then bind GTP and thereby, promotes the release of GEF. The selective binding of GTP instead of GDP is favored by the high cellular GTP/GDP ratio (Sprang 1997). In contrast, the conversion from the GTP to the GDP-bound states is an irreversible hydrolysis reaction (guanosine triphosphatase (GTPase) reaction). It is also intrinsically very slow, but can be accelerated by GTPase-Activating Proteins (GAPs).

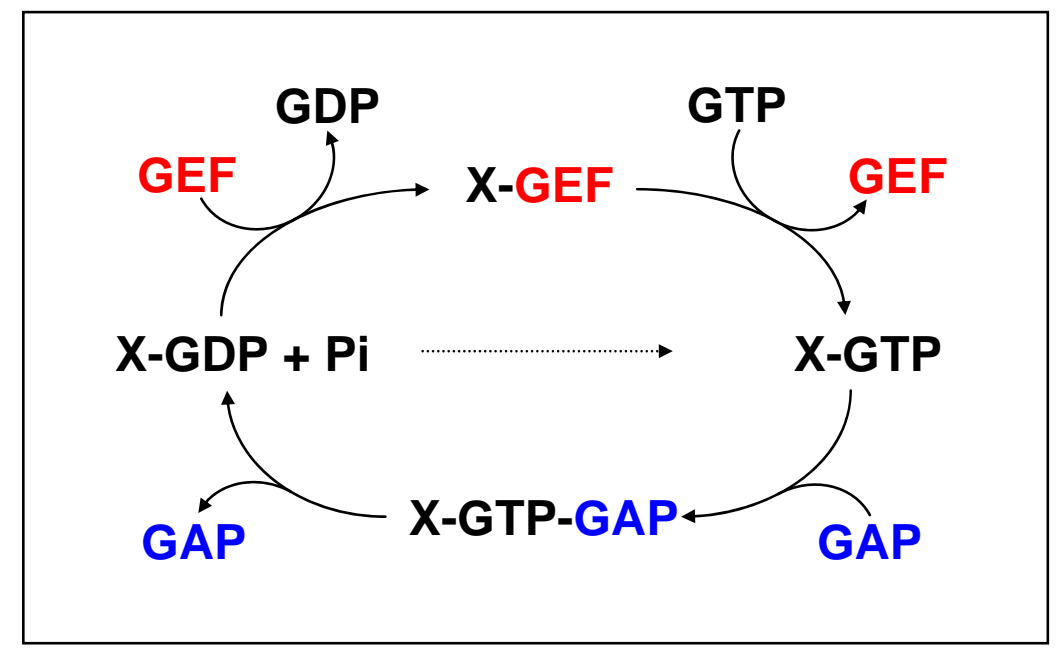

Figure 3. The mechanism of GTP/GDP cycling of guanine nucleotide-binding proteins. A GDP-bound protein exchanges its GDP for GEF, which in turn will be replaced by GTP. GTP-bound proteins hydrolyze GTP in a GAP-dependent mechanism (see main text for details).

Translation factor GTPases are multi-domain proteins. One of them, namely the Gdomain, is responsible for binding and hydrolyzing GTP. The structure of this domain is similar in all factor GTPases, comprising a $\beta$-sheet flanked by $\alpha$-helices. Accordingly, the mechanism of GTP binding is universal: a conserved N/TKXD motif binds the nucleotide base, while the P loop (phosphate-binding loop) interacts with the $\beta, \gamma$-phosphates of the 
nucleotide (Saraste et al. 1990). Structural studies showed that the switch apparatus itself is a conserved fundamental module, but that its regulators and effectors are quite diverse in structures and modes of interaction (Vetter and Wittinghofer 2001).

Two of the ribosomal GTPases, namely EF-Tu and RF3, were shown to conform to the canonical mechanism described for the GNBPs, while IF2 and EF-G seem to act differently. EF-Ts and the ribosome act as GEFs for EF-Tu and RF3, respectively, promoting their activation (Lucas-Lenard and Lipmann 1966; Zavialov et al. 2001). Unlike them, IF2 does not seem to discriminate between GTP and GDP, as it was shown to catalyze the initiation of translation in the presence of either nucleotide (Tomsic et al. 2000). The mechanism of nucleotide binding by EF-G, correlated to its function in translocation, is not entirely elucidated. The classical interpretation is that in solution, both GTP and GDP forms of EF-G are in equilibrium and it is not yet clear, which of these forms binds to the ribosome (Martemyanov et al. 2001; Hansson et al. 2005). Recently, it was hypothesized that the ribosome, in its pretranslocational state, would act as a GEF for EF-G:GDP (Zavialov et al. 2005). However, this hypothesis is challenged by the fact that EF-G:GDP has low affinity for the ribosome (Munishkin and Wool 1997). Kinetic analyses revealed that the binding of EF-G:GTP to the pretranslocation complex triggers rapid GTP hydrolysis. The energy of this process is used to drive the translocation. Thus, EF-G is in a GDP-bound conformation throughout the translocation and dissociates from the ribosome after this process is completed (Rodnina et al. 1997).

Based on these observations, it can be concluded that ribosomal GTPases act as molecular switches, cycling between their GTP- and GDP-bound states on the ribosome. They bind GTP and upon GTP hydrolysis undergo conformational changes. The structural changes modulate affinities of the factors for a particular functional state or for a ligand molecule they carry and thus, allow the entry into the next translation phase or the next step of the elongation cycle.

\section{E. The GAP function of the ribosome}

Translation factor-GTPases possess an intrinsic GTP hydrolysis activity in the presence of an appropriate physicochemical environment, but it does not meet the requirements of an efficient translational apparatus (De Vendittis et al. 1986). Importantly, this low intrinsic GTPase activity is stimulated by the presence of the empty ribosome 
(Kawakita et al. 1974). The amount of GTP hydrolyzed by EF-G in one minute was about three orders of magnitude higher in the presence of the E. coli ribosomes (Masullo et al. 1989). Furthermore, GTPase activity was augmented in the presence of tRNA-bound ribosome (Chinali and Parmeggiani 1982) and further increased when both elongation factors interacted with the ribosome (Mesters et al. 1994). Thus, in addition to its multiple functions during translation, the ribosome also induces an increase in factor GTP hydrolysis by several orders of magnitude and thereby possesses a GAP function.

Biochemical analyses have defined a region of the large ribosomal subunit, whose elements act as GAPs for EF-Tu, EF-G, IF2 and RF3. This region encompasses several rRNA and r-protein elements, namely the sarcin-ricin loop, L10/L11 binding region, proteins L6, L11, L14 and the L7/L12 stalk. The latter corresponds to an extended feature of the 50S ribosomal body, comprising multiple copies of protein $\mathrm{L} 7 / \mathrm{L} 12$, which are linked to the ribosomal rRNA through protein L10. The entire region formed by rRNAs and r-proteins can be collectively termed the GTPase Associated Region (GAR) or factorbinding site (Wimberly et al. 1999) (Figure 4).

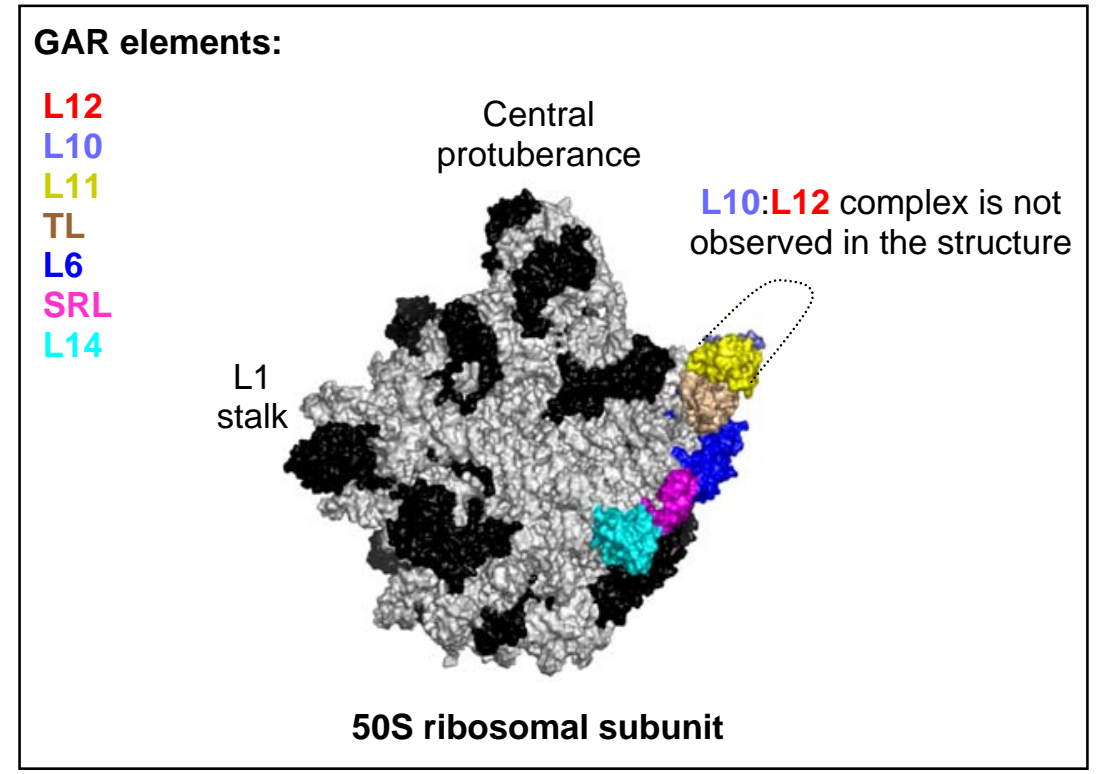

Figure 4. The GTPase associated region (GAR or factor binding site) of the large ribosomal subunit. 50S is depicted with its major landmarks: the central protuberance, the L1 stalk and the L7/L12 stalk (lacking the peripheral elements of the L10:L12 complex); rRNAs are depicted in gray and r-proteins in black, respectively. Components of the GTPase associated region (GAR) are highlighted in different colors and denominated accordingly in the legend on the left; TL, thiostrepton loop or L10/L11 rRNA binding region; SRL, sarcin-ricin loop (adapted from (Ban et al. 2000), PDB accession code 1FFK).

Initially, the complex of L11 protein with its corresponding rRNA binding region of the 23S rRNA (L11-rRNA region) was defined as the GTPase Associated Center 
(GAC). However, there is no uniform terminology of GAC in the literature, as it was also used to define, both the L11-rRNA region and the neighboring sarcin-ricin loop.

The GTPase associated region (GAR) acts as a ribosomal GAP. From the analysis of other GAP family members, three putative models emerge, to account for the GAP ribosomal function (reviewed in (Vetter and Wittinghofer 1999)):

- model 1: as for RasGAP and RhoGAP, the ribosome may provide a catalytic arginine residue ("arginine finger") to the GTPases' active sites and thereby stabilize their GTP transition state, leading to an increase in the rate of GTP hydrolysis (Noel 1997)

- model 2: as for RGSs (regulators of G protein signaling), the ribosome may bind to the switch regions of the factor GTPases and stabilize the GTPase transition state (Hunt et al. 1996)

- model 3: as for ARFGAP, the ribosome may enhance GTP hydrolysis by interacting with distant regions of the nucleotide binding site (Goldberg 1999)

Despite the definition of a ribosomal region capable of assuming the GAP function, the discrimination between either models remained so far unclear.

\section{Sarcin-ricin loop, proteins L6 and L14}

The Sarcin-Ricin Loop (SRL or stem-loop 95 or ribotoxin loop) is a universally conserved stem-loop structure, located in domain VI of the $23 \mathrm{~S}$ rRNA around position 2660 (nucleotides 2645-2675 in E. coli 23S rRNA). The SRL is essential for the binding of elongation factors (Moazed et al. 1988) and IF2 (La Teana et al. 2001), and possibly acts as a stimulator of their GTPase activity. Additionally, it was shown that the SRL is the target of ribotoxins $\alpha$-sarcin (Endo and Wool 1982) and ricin (Endo et al. 1987). The cleavage of a single covalent bond by either toxin inhibits the binding of EFs to the ribosome, thereby inactivating translation (Montanaro et al. 1975; Fernandez-Puentes and Vazquez 1977). Crystallographic data of the 50S ribosomal subunit (Ban et al. 1999; Ban et al. 2000) presented a detailed model of the structure of the translation factor-binding site. In these studies, the first element fitted into the electron density of the factor binding site was the SRL, which has a typical S-shaped structure (Szewczak and Moore 1995; Correll et al. 1998). Cross-linking (Leffers et al. 1988; Urlaub et al. 1995; Uchiumi et al. 1999) and immunoelectron microscopy (Walleczek et al. 1988) approaches provided information concerning other elements neighboring the SRL, namely proteins L6, L14 and the L7/L12 stalk. Notably, by docking the ternary complex (EF-Tu:GTP:tRNA) and EF- 
G:GDP on the 50S subunit, interactions between the G domains and the GAR components were predicted (Ban et al. 1999). The C-terminal domain of protein L6 was found in the vicinity of both EF-G and EF-Tu G domains. Protein L14 was assumed to make extensive contacts with domains 2 and 3 of EF-G, EF-Tu and the acceptor stem of tRNA carried by EF-Tu (Ban et al. 1999). Additionally, G domains of both EF-Tu in the GTP conformation and EF-G in the GDP conformation seemed to contact the ribotoxin loop. Moreover, their switch regions, whose conformations are modified upon GTP hydrolysis, were found sandwiched between L14 and the SRL (Ban et al. 1999). The latter has a central position in the factor binding site, suggesting that it stimulates the GTPase activity of the ribosomebound factors.

Supplementary evidence supporting these crystallographic data came from a cryoEM map of EF-G bound to the 70S ribosome (Wriggers et al. 2000) that showed a direct contact between the $\mathrm{G}$ domain of the factor and sarcin-ricin loop. Moreover, a $13 \AA$ cryoEM three-dimensional reconstruction of the EF-Tu:GTP:aa-tRNA ternary complex stalled with kirromycin following GTP hydrolysis (Stark et al. 2002), depicted an extensive interaction between the G domain of EF-Tu and the SRL. This contact involves the switch regions of EF-Tu (nucleotide-binding pocket and the effector loop), implicating the SRL in the GTPase activation of this factor. IF2:GTP was visualized by cryo-electron microscopy in the proximity of the sarcin-ricin-loop. In contrast, IF2:GDP was found distant from the SRL and oriented toward protein L6 (Myasnikov et al. 2005). Interestingly, RF3 in its GTP-bound form adopted two conformations: in state-1, RF3 made only few contacts with the ribosome, predominantly with the $30 \mathrm{~S}$ subunit; in state-2, RF3 was tightly bound to the ribosome and its $\mathrm{G}$ domain was oriented towards the factor binding site, suggesting an interaction similar to other factor GTPases (e.g. EFs) (Klaholz et al. 2004).

\section{L11 protein and L10/L11 rRNA binding region}

Protein L11 and L10:L12 complex interact in a mutually cooperative manner with a short sequence within domain II of 23S rRNA (L10/L11 rRNA binding region) (Beauclerk et al. 1984). L10:L12 elements form an elongated protuberance in the large ribosomal subunit, called the L7/L12 stalk. Early immunoelectron microscopy experiments placed protein L11 at the base of the L7/L12 stalk (Tate et al. 1984). The L10/L11 rRNA region neighbors the sarcin-ricin loop. Both rRNA elements display a high degree of 
conservation throughout the kingdoms, suggestive of their important role in the translation process.

L11 interacts with a fragment of 58 nucleotides (1051-1108 in E. coli) within the L10/L11 rRNA region forming the L11:rRNA complex (Thompson et al. 1979; Schmidt et al. 1981). This complex is the target of a family of thiazole antibiotics, including thiostrepton and micrococcin. As a consequence, the L10/L11 rRNA sequence is often referred to as the Thiostrepton Loop (TL). These drugs bind irreversibly to the L11:rRNA complex and inhibit protein biosynthesis. The structure of thiostrepton was determined few decades ago (Hensens et al. 1983), but its exact orientation within the L11:rRNA complex is still unknown. However, based on structural and biochemical data, it was proposed that the thiostrepton/micrococcin-binding site is located in a "pocket" formed by the 1067/1095 region of the 23S rRNA and a proline-rich helix in the NTD of L11 (Wimberly et al. 1999). A proline 22 in the NTD of the L11 from bacteria, crucial for thiostrepton binding, is not conserved in eukaryotic counterparts, consistent with the natural resistance of the latter to thiazole antibiotics. Thus, the structural basis for the interaction of the L11:rRNA complex with these antibiotics (previously shown to be effective agents against the malaria parasite Plasmodium falciparum) is of great medical interest. The mechanism by which thiazoles arrest protein synthesis was studied in more detail for EF-G. Micrococcin inhibits a number of processes believed to involve the ribosomal A site while stimulating GTP hydrolysis by EF-G (Cundliffe and Thompson 1981). Thiostrepton was originally regarded as an inhibitor of GTP hydrolysis by EF-G (Pestka 1970). Later experiments showed that the drastically decreased GTPase activity of EF-G is an indirect effect of thiostrepton binding to the ribosome, which in turn reduces the affinity of EF-G for the ribosome (Cameron et al. 2002). Moreover, kinetic experiments demonstrated that thiostrepton does not prevent GTP hydrolysis by EF-G, but rather affects subsequent steps, such as inorganic phosphate and EF-G release (Rodnina et al. 1999). A possible explanation for this effect could be that thiostrepton prevents the mobile L11 NTD to undergo a conformational change required after GTP hydrolysis, thus blocking translation in this stage. Consistently, thiostrepton was used to visualize conformational changes occurring after GTP hydrolysis during translocation. Thus, it was seen that EF-G was trapped by thiostrepton at an earlier stage than fusidic acid, the latter preventing the EF-G:GDP dissociation from the ribosome (Stark et al. 2000). 
The high-resolution structure of the L11:rRNA complex revealed a compact organization of the rRNA (Wimberly et al. 1999). Protein L11 consists of two globular domains connected by a short, proline-rich linker, which allows the independent movement of the NTD with respect to the CTD. These two domains are unequally associated with rRNA: the C-terminal domain (CTD) forms extensive tight contacts with the $58 \mathrm{nt}$ fragment of the 23S rRNA, while the N-terminal domain (NTD) interacts weakly with this region. Contacts between the CTD of L11 and rRNA involve mainly the protein backbone and rRNA 2' OH moieties, suggesting a case of protein-RNA recognition based on shape complementarity.

The NTD region of L11 could not be visualized in the high-resolution map of the $50 \mathrm{~S}$ subunit, or in several cryo-EM maps of the ribosome, most probably due to its flexibility (Ban et al. 2000). However, an intermediate resolution structure of the large ribosomal subunit placed the 58 nt-rRNA fragment and the CTD of L11 in the electron density and offered a convincing model for the location of the NTD (Ban et al. 1999). This hypothesis was further supported by a cryo-electron microscopy analysis of the GDP stateEF-G modeled onto the 70S ribosome, which ascertained the location of the L11-NTD in the electron density map (Agrawal et al. 2001). Noteworthy, this latter study also revealed that, following GTP hydrolysis, an Arc-Like Connection (ALC) is formed between the L11 NTD and the G' domain (a subdomain of the G domain) of EF-G. This thin bridge was noticed in both GDP state-EF-G and EF-Tu, but not in their GTP conformations, in several other cryo-EM studies (Stark et al. 1997b; Agrawal et al. 1998; Agrawal et al. 1999; Stark et al. 2000; Agrawal et al. 2001). In these reports, the G or G' domain of the GDP-state factors were found to contact a lobe positioned at the base of the stalk, which, as Agrawal and coworkers have inferred, implicates the N-terminal domain of protein L11.

The interaction of the L11:rRNA region with EF-Tu is less understood. Two similar cryo-EM studies of the kirromycin-stalled ternary complex (EF-Tu:GTP:tRNA) concluded that upon the cognate codon recognition, the tRNA interacts with the GTPase associated center and triggers GTP hydrolysis by EF-Tu (Stark et al. 2002; Valle et al. 2002). However, in these reports, different interpretations were made regarding the interaction between tRNA and GAC elements. The first study suggested an interaction between the tRNA and protein L11, whereas SRL would stimulate GTP hydrolysis. This observation portrayed protein L11 as a modulator of the GTPase rate, rather than a direct player in this process (Stark et al. 2002). Conversely, the second report assigned the tRNA 
with an essential role in GTPase activation, interacting with the L11 rRNA binding region (Valle et al. 2002). It was envisioned that when a ternary complex binds the ribosome, the G-domain of EF-Tu contacts the SRL and the tRNA interacts with the L11 binding region of rRNA (around nucleotide 1067), which is in an "opened" conformation. Successful codon-anticodon base-pairing goes hand in hand with the interaction between the tRNA (T-loop) and the L11 binding lobe of rRNA, stabilizing it in a "closed conformation". This event is followed by GTP hydrolysis and by the transition of the rRNA to the initial “opened" conformation (Valle et al. 2003b).

\section{L10:L12 complex}

\section{a. Characterization of the L10-L12 interaction}

The most studied element of the stalk, but at the same time still puzzling, both in structure and function, is the L7/L12 protein (reviewed in (Gudkov 1997; Wahl and Moller 2002)). Together with protein L10, it builds a lateral protrusion on the large ribosomal subunit, termed the L7/L12 stalk (Figure 4). Indeed, early studies involving electron microscopic visualization of antibody-labeled ribosomal subunits, evidenced the presence of protein L7/L12 on the "rod-like appendage" (or stalk) of the 50S subunit (Boublik et al. 1976; Lake 1976; Strycharz et al. 1978; Kastner et al. 1981).

First considered as an individual protein, named L8, by means of two-dimensional gel electrophoresis (Kaltschmidt and Wittmann 1970), L10 and L7/L12 were further identified as distinct proteins forming a stable complex (Pettersson et al. 1976). The assignment L7/L12 resides in the occurrence, in different culture conditions, of two similar acidic protein populations, L12 and L7, the only difference between them being the Nterminal acetylation of L7 (Deusser 1972); for simplicity, in the following, the L7/L12 protein will be referred to as L12.

Both extraction from the ribosome or in vitro purification of the E. coli L10:L12 complex, yielded a pentameric complex formed by one copy of L10 and four copies L12, the latter being the only multicopy ribosomal protein (Terhorst et al. 1973; Hardy 1975; Subramanian 1975; Pettersson and Liljas 1979).

The L10:L12 complex is anchored on the large ribosomal subunit via protein L10. The L10 N-terminal domain (NTD) binds to a highly conserved region of the 23S rRNA (L10/L11 binding region), overlapping the binding site of protein L11 (Beauclerk et al. 1984; Egebjerg et al. 1990). In turn, four copies of protein L12 associate as two dimers 
with the C-terminus of L10 (CTD). Terminal deletion variants of ribosomal protein L10 were constructed in order to define the binding sites of the two L12 dimers. Thus, it was determined that the deletion of the last ten amino acids of L10 results in the loss of one L12 dimer, while the deletion of the last 20 amino acids led to the loss of both dimers (Griaznova and Traut 2000) (Figure 5).

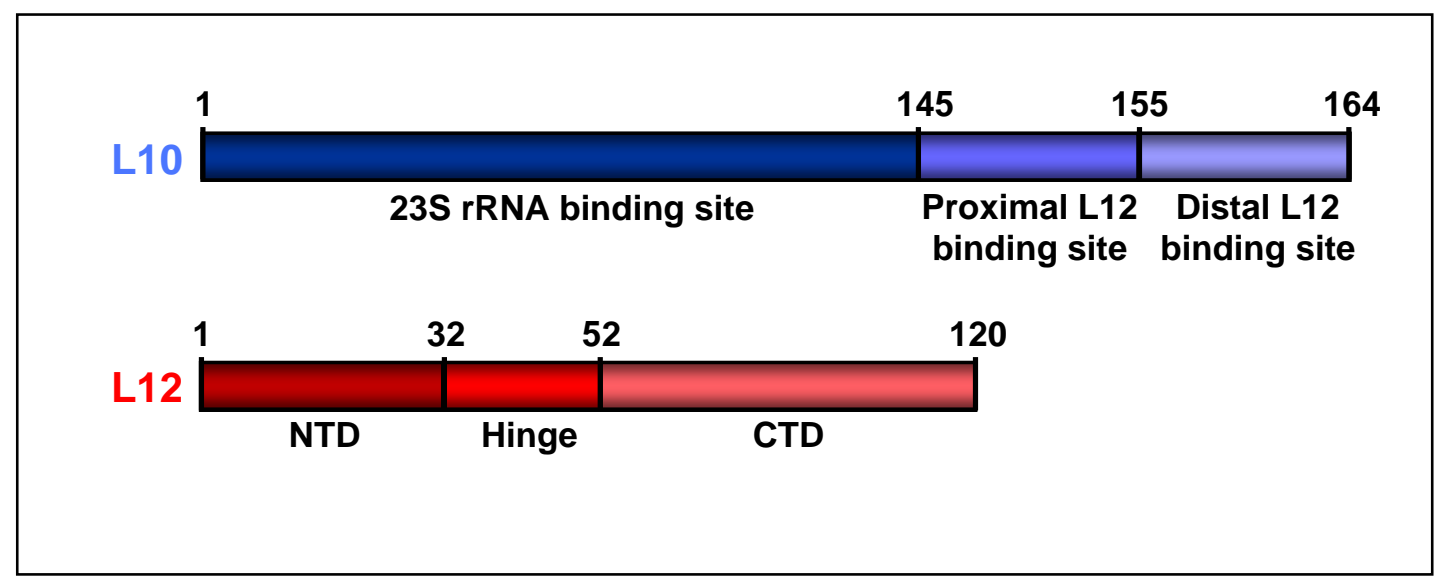

Figure 5. Organization of L10 and L12 proteins from E. coli. L10 binds to the 23S rRNA via its N-terminal part (residues 1-144), whereas the C-terminal part (residues 145-164) is responsible for the interaction with L12. One L12 protein encompasses three domains: a NTD (residues 1-31) connected to the CTD (residues 52-120) through a linker (hinge).

\section{b. Domain organization and dimerization mode of the L12 protein}

Several biochemical, biophysical and structural data further deciphered the organization of the L12 protein. Three structurally distinct regions underlie L12 function: a $\mathrm{N}$-terminal domain responsible for both dimerization and interaction with L10 (Gudkov and Behlke 1978; Gudkov et al. 1980), a C-terminal domain involved in factor binding and stimulation of their GTPase activity (Kischa et al. 1971), and a flexible hinge connecting both domains (Liljas and Gudkov 1987) (Figure 5). NMR studies envisioned that the NTD dimers of L12 interact in an antiparallel fashion, forming a four helix bundle (Bocharov et al. 1996). The CTDs have a globular aspect and exhibit on their surface several conserved residues (Liao and Dennis 1994) believed to represent the sites of interaction with translation factors (Wieden et al. 2001). The high-resolution crystal structure of the L12 Cterminal domain - the first structure of a ribosomal component - displays a split $\beta-\alpha-\beta$ motif, identified as an RNA recognition motif (RRM), although in this particular case no interaction with the rRNA was observed (Leijonmarck et al. 1980). Proton magnetic resonance studies portrayed the hinge region as highly mobile (Bushuev et al. 1989). The 
importance of the hinge in translation was acknowledged by experiments with deletion mutants lacking this domain, which yielded ribosomes completely inactive (Oleinikov et al. 1993; Dey et al. 1995). In addition, it was hypothesized that the flexibility of the hinge may cause independent movements of the L12 CTDs. Indeed, a cryo-EM structure of the $70 \mathrm{~S}$ ribosome, displaying a nanogold labeled CTD of L12, localized this domain at different sites of the ribosome, such as the stalk base, the peptidyl transferase center or the head of the 30S subunit (Montesano-Roditis et al. 2001). The latter study was supported by cross linking (Dey et al. 1998) and NMR data (Mulder et al. 2004).

L12 proteins, both in solution or in complex with L10, form symmetrical dimers via their N-terminal domains (Gudkov and Behlke 1978). Various arrangements of the subunits in L12 dimers have been proposed, i.e. antiparallel (head-to-tail orientation) (Moller et al. 1972) or parallel (Liljas 1982). The latter model is favored by experiments in which the hydrogen peroxide oxidation of methionine residues present in the NTD caused the disruption of the dimer and failure of L10 binding (Gudkov et al. 1977). Taken together, these data support a model of a parallel dimer in which the CTDs are well separated from the NTDs via a highly mobile linker.

\section{c. Structures of the L12 protein}

The crystal structure of the isolated L12 protein from Thermotoga maritima challenged the previous model of the association of L12 dimers, providing new exciting hypotheses concerning both domain organization and dimerization (Wahl et al. 2000a). The asymmetric unit comprised two full-length molecules and two proteolysed $\mathrm{N}$-terminal fragments, forming a compact hetero-tetrameric structure. The structure exhibited contacts from each component part to every other, except for an interaction between the NTD fragments. Two dimerization modes were observed: parallel, between the two full-length monomers, which form a tight, symmetric "core dimer", and antiparallel, between each Nterminal domain of a full-length monomer and an N-terminal L12 fragment. The hinge was seen in two alternative conformations: an extended coil in the NTD fragments or a long $\alpha$ helix that folds back on the N-terminal domain of the full-length molecule. The existence of both an antiparallel dimerization mode and an unstructured, extended hinge was shown for isolated E. coli L12 (ecoL12) in solution by NMR (Bocharov et al. 2004) and for $T$. maritima L12 (tmaL12) in solution by FRET experiments (Moens et al. 2005). The latter study also indicated that tmaL12 proteins formed dimers in solution, which implied that 
the tetrameric arrangement observed in the crystal structure does not represent the solution state of the protein. Thus, corroborating the observations made for, both L12 in solution and in the crystalline environment, the ribosome bound-L12 was assumed to display one monomer with an $\alpha$-helical hinge, and one with an extended hinge, allowing the movement of the CTD (Chandra Sanyal and Liljas 2000; Bocharov et al. 2004; Mulder et al. 2004; Moens et al. 2005).

\section{d. Different locations of the L12 protein on the ribosome}

In the past two decades, several hypotheses were made regarding the orientation of L12 molecules within the ribosome. Thus, it was shown that one dimer per particle was sufficient to form a visible stalk (Moller et al. 1983), despite earlier studies using polyclonal antibodies that suggested that both dimers were present in the stalk (Tokimatsu et al. 1981).

By chemical cross-linking studies it was proposed that a dimer could reach the EF$\mathrm{Tu}$ binding site on the 30S subunit (Dey et al. 1998). Additionally, three possible alternative locations of the dimer on the 50S subunit were inferred: (i) accounting for the stalk protrusion (ii) bent at the base of the stalk, near the EF-G binding site; (iii) extended far across the body of the 50S subunit, as the CTD of L12 was shown to be engaged in interactions with L2 and L5. The latter location can be refuted, since this site could only be reached by a twice longer L12 dimer (Wahl and Moller 2002).

\section{e. Phylogenetic comparison}

Similar L10:L12 complexes are also present in archaeal and eukaryotic ribosomes (reviewed in (Gonzalo and Reboud 2003)). Both archaeal (aL10 or L10E) and eukaryotic (P0) L10 are longer than their bacterial counterparts. In archaea, there is only one form of the L12-like protein. In eukaryotes, the bacterial L12 is replaced by two proteins, P1 and P2 (Wood 1991), which form further subgroups in yeast (Shimmin et al. 1989) and by three proteins in plants (P1, P2, P3) (Szick et al. 1998). Dimers of both P1 and P2 are anchored to the ribosome via P0. The mechanism by which L12-like proteins exert their function on the ribosome is different: while bacterial proteins seemed permanently associated to core particles, P-proteins (post-translational Phosphorilated proteins) were found interchangeable between ribosome-bound and cytoplasmic non-phosphorilated pools (Kopke et al. 1992). 
The bacterial stalk proteins do not have a detectable sequence homology to the eukaryotic and archaeal counterparts, which, on the other hand, are clearly homologous to each other (Liljas 1991). The replacement of the L10:L12 complex in E. coli ribosomes with the rat $\mathrm{P}$ protein complex changed its specificity from prokaryotic elongation factor (EF-G) binding to eukaryotic EF-2-dependent GTPase activity (Uchiumi et al. 1999). However, the latter study suggested that the rRNA-L10 protein association seems to be conserved across kingdoms, consistent with several other functional hybrid ribosomes reported: yeast cores reconstituted with E. coli L12 (Sanchez-Madrid et al. 1981) and vice versa, (Wool and Stöffler 1974), as well as E. coli ribosomes reconstituted with an archaeal P complex (Nomura et al. 2006)).

\section{f. Translation factor-related functions}

An interesting property of both L10:L12 complex or isolated L12, allowing thorough studies of their functions, is represented by their selective extraction from the ribosome following a specific high salt/ethanol treatment (Kischa et al. 1971; Pettersson and Kurland 1980). The subsequent addition of these proteins to the depleted core particles, results in functional ribosomes. Thus, it was noticed that a severely impaired GTPase activity by EF-G in L12-depleted ribosomes, was rescued by addition of the purified protein (Kischa et al. 1971). Similar effects were seen for the other soluble factor GTPases EF-Tu, IF2, RF3 (reviewed in (Brot and Weissbach 1981)). The same observations were made with ribosomes depleted of the entire pentameric complex (Hamel et al. 1972). Based on these investigations, it was suggested that L12 is directly involved in translation factor GTPases binding and stimulation of their GTP hydrolysis (Kischa et al. 1971; Fakunding et al. 1973; Brot et al. 1974). Numerous other studies, including sitedirected mutagenesis, limited proteolysis, crosslinking or structural approaches supported this hypothesis (Gudkov 1997; Wahl and Moller 2002).

Noteworthy, factor binding was evidenced not only for the ribosome-bound L12, but also for the protein in isolation. However, in the latter case, a different outcome was observed for elongation factor-dependent GTP-hydrolysis: while EF-G-dependent GTP hydrolysis was strongly stimulated by L12 (Savelsbergh et al. 2000), no GTPase activity by EF-Tu was detected, indicating that additional ribosomal components are required for this purpose (Piepenburg et al. 2000). Accordingly, recent rapid kinetics and mutagenesis experiments evidenced that the ribosome-bound L12 promotes EF-Tu binding (Kothe et al. 
2004). Furthermore, comparisons of L12 CTD and EF-Ts (the guanine-nucleotide exchange factor of EF-Tu) structures revealed that the L12 CTD interacts with helix D of EF-Tu (Wieden et al. 2001); consistently, the corresponding region of the L12 CTD was found essential for the initial binding of EF-Tu to the ribosome (Kothe et al. 2004).

\section{Rationales}

Numerous components of the GTPase associated region (GAR) have been ascribed in the recent crystal structures of the bacterial ribosome (Figure 4). However, the structure of L10:L12, a protein complex shown to be instrumental in translation, has long remained elusive. Several lines of evidence have demonstrated that this complex suffers major rearrangements as a result of its interactions with elongation factors during protein synthesis. The difficulty of disclosing electron density corresponding to this region most probably stems from its inherent conformational dynamics and potential heterogeneity. Thus, the L10:L12 structure was found disordered or absent even in high-resolution crystal structures of 50S ribosomal subunit (Ban et al. 2000) (Figure 4) or of entire ribosomes (Schuwirth et al. 2005) (Figure 2).

In a crystal structure of the 70S ribosome (Yusupov et al. 2001), one of the L12 dimers was tentatively modeled within the electron density in its compact arrangement, as previously described for L12 in isolation (Wahl et al. 2000a). However, this ribosome structure did not attribute any electron density for the second dimer of L12 or for the L10 protein. Two helices of the latter were defined in a high-resolution structure of the large ribosomal subunit (Ban et al. 2000). They correspond to residues located at the N-terminal part of L10 that interact with a secondary structure motif, called the K-turn (Klein et al. 2001). Hence, at the moment, there is no crystal structure describing a full L10:L12 complex and its location on the ribosome.

Cryo-EM studies evidenced that the $G$ domains of elongation factors in their GDP-like conformation form a bridge with the base of the stalk (Figure 6A, C) (Stark et al. 1997b; Agrawal et al. 2001), which was not observed in their GTP-bound states (Figure 6B) (Agrawal et al. 1999). Moreover, the G domains of other factor GTPases, such as IF2 (Allen et al. 2005) and RF3 (Klaholz et al. 2004) seem to interact with several components of the GTPase associated region (GAR) in a site delineated by the sarcin-ricin loop and the 
stalk. However, for protein L12, a central element of the GAR, it is not clear how it participates in GTP hydrolysis, since a contact between L12 and elongation factors, or other components of the ribosome that could trigger this process was not evidenced in previous structures or cryo-EM studies.

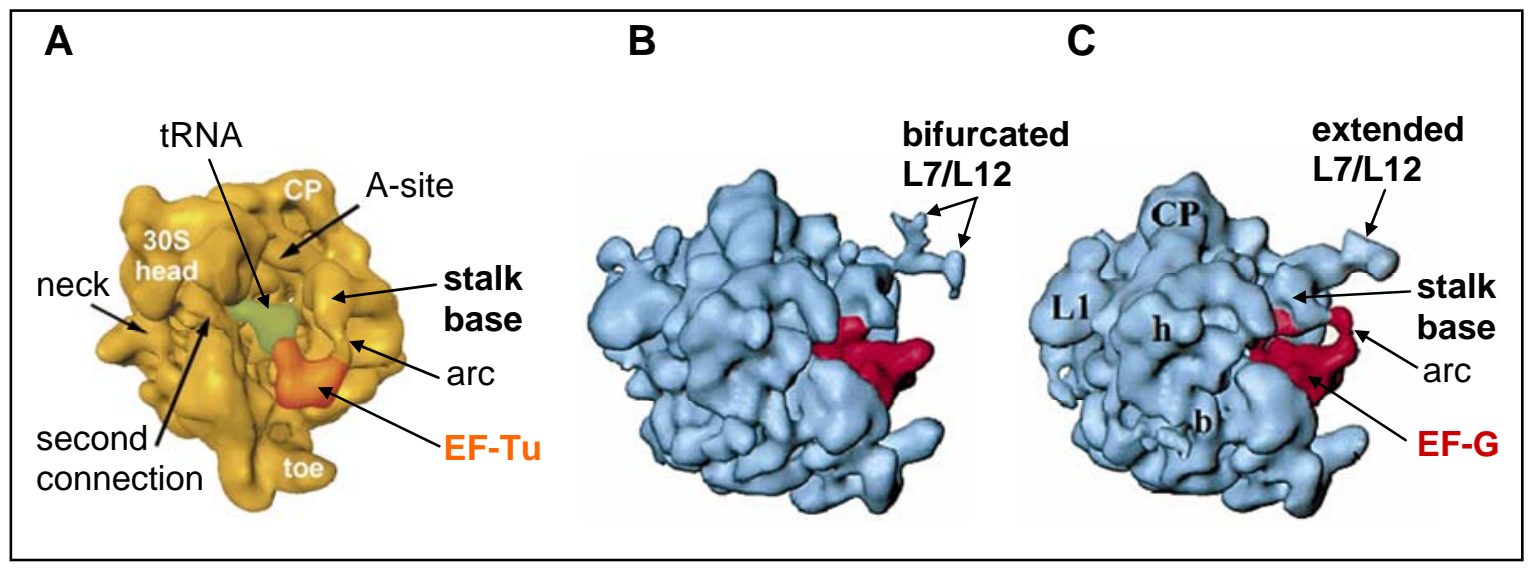

Figure 6. Visualization of the L7/L12 stalk by cryo-electron microscopy and single particle reconstruction at several main steps of the elongation cycle. (A) Ternary complex (EF-Tu:Phe:tRNA) kirromycin-locked at the A site (reproduced from Stark et al., 1997). The main landmarks of the 30S (head, neck, toe) and of the 50S subunit (central protuberance, CP) are evidenced. The stalk base forms an arc-like connection with EF-Tu. (B) EF-G (dark red) is bound to the ribosome in complex with a non-hydrolysable GTP analog. In this particular step, the L7/L12 stalk elements accounting for the L10:L12 protein complex adopt a bifurcated shape (reproduced from Agrawal et al., 1999). (C) EF-G is bound to the ribosome in the presence of fusidic acid, in a GDP-like conformation. Main landmarks of the 30S subunit: head (h), body (b) and 50S subunit: central protuberance $(\mathrm{CP}), \mathrm{L} 1$ protuberance $(\mathrm{L1})$, are depicted. Contrary to $(\mathrm{B})$, here the stalk elements accounting for the L10:L12 complex are seen in an extended conformation. Similarly to (A), an arc-like connection is observed between the G'-domain of EF-G and the stalk base (reproduced from Agrawal et al., 1999).

In the 70S:EF-G:GDP:fusidic acid complex (Figure 6C), an extended lateral protrusion was observed, which became bifurcated in the GTP state of the same complex (Figure 6B) (Agrawal et al. 1999) and presumably represented the L10:L12 complex. However, these elements cannot be reliably interpreted without an atomic structure of the L10:L12 complex. Therefore, a crystallographic study of this complex is required to disclose the L10 protein structure and to understand how it distinguishes and accommodates two L12 dimers in its asymmetric sequence (Wahl and Moller 2002). In addition, this structure could reveal how this complex is anchored on the ribosome, what is the in situ orientation of the L12 dimers (particularly, how many copies of L12 form the extended stalk) and which parts of this complex are flexible when bound to the ribosome. The L10:L12 complex structure could be regarded as one of the last pieces to be discovered in the jigsaw puzzle of the ribosome and its deciphering would aid to further understand the complex molecular mechanism of translation. 
Here, a high-resolution crystal structure of the L10 protein in complex with the N-terminal domain of L12 from the hyperthermophilic bacterium Thermotoga maritima, in three different crystal forms, is reported. Attempts to solve the structure of the full-length L10:L12 protein complex were also made. The fitting of the resulting L10:L12 NTD complex in situ on the $50 \mathrm{~S}$ ribosomal subunit is discussed. The morphology and dynamics of the L7/L12 stalk region, as seen in electron microscopic reconstructions of ribosomes is reinterpreted. Together with structures of the isolated L12, a complete atomic model of the stalk is devised. Based on this model, structure-function relationships were established. 


\section{Materials and Methods}

\section{Molecular cloning}

\section{A. Genomic DNA preparation}

T. maritima MSB8 cells were purchased from DSMZ (Braunschweig) and their genomic DNA was isolated by phenol-chloroform extraction. Briefly, cells were resuspended in TE buffer (10 mM Tris- $\mathrm{HCl}, 1 \mathrm{mM}$ EDTA, $\mathrm{pH}$ 8.0) and mixed with one volume of phenol (Roth, Karlsruhe). After centrifugation, one volume of chloroform (Roth) was added to the upper phase and re-centrifuged. The DNA content in the resulting upper phase was extracted by precipitation with 3 volumes of $100 \%$ ethanol containing $10 \%$ of $3 \mathrm{M}$ sodium acetate $\mathrm{pH} 5.5$, and was subsequently placed at $-80^{\circ} \mathrm{C}$ for $20 \mathrm{~min}$. Following $30 \mathrm{~min}$ centrifugation, the DNA pellet was washed with $70 \%$ ethanol and resuspended in $100 \mu \mathrm{l}$ of water. The concentration, measured at $\mathrm{OD}_{260}$ (Sambrook et al. 1989), was $97.5 \mathrm{ng} / \mu 1$.

\section{B. PCR amplification}

The DNA fragments encompassing the entire coding sequence of the proteins used in this work were amplified by PCR. For this purpose, primers (MWG Biotech, Ebersberg) were designed to introduce restriction enzyme sites compatible with those present in the multiple cloning site of the vectors used (Table 4). 4-9 bases were added at the 5' ends of these primers, to allow an optimal activity of the restriction endonucleases. The following describes a typical PCR reaction and a PCR cycling program employed for the amplification of the products generated in this work (the only variable was the annealing temperature, which was chosen based on the melting temperature of the primers).

$\underline{50 \mu \mathrm{l} \text { PCR reaction mixture }}$

$5.0 \mu 1 \quad 10 \mathrm{x}$ cloned $P f u$ buffer

$5.0 \mu \mathrm{l} \quad$ DNA sample (200 ng)

$1.0 \mu \mathrm{l} \quad 5$, primer $(50 \mathrm{pmol} / \mu \mathrm{l})$

$1.0 \mu \mathrm{l} \quad 3$ ' primer $(50 \mathrm{pmol} / \mu \mathrm{l})$

$5.0 \mu \mathrm{l} \quad$ DMSO

$2.0 \mu \mathrm{l} \quad \mathrm{dNTP}(25 \mathrm{mM}$ each $)$

$29.0 \mu \mathrm{l} \quad \mathrm{H}_{2} \mathrm{O}$

$2.0 \mu \mathrm{l} \quad P f u$ polymerase $(5 \mathrm{U} / \mu \mathrm{l})(\mathrm{NEB})$
PCR program

$1 \mathrm{x} \quad 94^{\circ} \mathrm{C} \quad 2 \mathrm{~min}$

$35 \mathrm{x} \quad 94^{\circ} \mathrm{C} \quad 1 \mathrm{~min}$

$55^{\circ} \mathrm{C} \quad 1 \mathrm{~min}$

$72^{\circ} \mathrm{C} \quad 3 \mathrm{~min}$

$1 \mathrm{x} \quad 72^{\circ} \mathrm{C} \quad 5 \mathrm{~min}$

hold temperature at $4^{\circ} \mathrm{C}$ 


\begin{tabular}{|c|c|c|c|}
\hline Gene product & Sequence $\left(5^{\prime} \rightarrow 3^{\prime}\right)$ & $\begin{array}{l}\text { Restriction } \\
\text { enzyme }\end{array}$ & Vector \\
\hline \multirow[t]{2}{*}{ aae L10 } & F: CGATGCCATGGCTGAATTTGACAAGGAAGCTTAC & NcoI & pETM-CoEx \\
\hline & R: CGACGGTACCTTACTGACCTCCTTTAGATTTTTCTTC & Acc65I & \\
\hline \multirow[t]{2}{*}{ aae L12 } & F: CGATGCCATGGCAACTTTAACTATTGACGAG & NcoI & pETM-CoEx \\
\hline & R: CGACGGTACCTTACTTGAGCTCGACTTCCGCTC & Acc65I & \\
\hline \multirow[t]{2}{*}{ tmaL10 } & F: CGTACGTCTCACATGCTGACCAGGCAACAGAAAG & BsmBI & pETM-ZZ \\
\hline & R: CGACGGTACCTCATTCAGATTTTTTCTCTTTAATAGC & Acc65I & \\
\hline \multirow[t]{2}{*}{ tmaL12 CTD } & F: GCTGTACAGCATATGACAGAGTTTGACGTCGTTTTG & NdeI & $\mathrm{pETM} 22 \mathrm{~b}(+)$ \\
\hline & R: CTAATTGGATCCTTACTTCAGTTCCACTTCAGCACC & BamHI & \\
\hline \multirow[t]{2}{*}{ tmaL12 CTD } & F: GATACGTCTCACATGACAGAGTTTGACGTCGTTTTG & BsmBI & pETM-11 \\
\hline & R: CTGAACTATGGTACCTTACTTCAGTTCCACTTCAGC & Acc65I & \\
\hline \multirow[t]{2}{*}{ tmaEF-Tu (Gd) } & F: GTTGTACAGCATATGGCGAAGGAAAAATTTGTGAGAAC & NdeI & pETM22b(+) \\
\hline & R: CTCAGTGGATCCTTAATCAGGAATGTAGTTATCCATAG & BamHI & \\
\hline \multirow[t]{2}{*}{ tmaEF-Tu (Gd) } & F: GTATCACCATGGCGAAGGAAAAATTTGTGAGAAC & NcoI & pETM-11 \\
\hline & R: CGTAGTGGTACCTTAATCAAGAATGTAGTTATCC & Acc65I & \\
\hline \multirow[t]{2}{*}{$\operatorname{tma\mathrm {L}11}$} & F: GATTATCCATGGCAGAGAAAGTAGCGGCTCAG & NcoI & pETM-11 \\
\hline & R: CCTGTCGGTACCTCAGTCCACTACTTCTATTC & Acc65I & \\
\hline
\end{tabular}

Table 4. Oligonucleotide primers used for PCR amplification. The encoded gene product names, primers used, restriction sites inserted within the primers (also in red) and the vectors used for targeted cloning are indicated. For each primer pair, the forward primer is denominated $\mathrm{F}$ and the reverse $\mathrm{R}$, respectively.

\section{Restriction digestion}

Following PCR, the products of amplification were purified with the GFX purification kit (Amersham Biosciences, Freiburg) and subjected to digestion with appropriate restriction endonucleases, according to the manufacturer's instructions (New England Biolabs, Frankfurt). The enzymes used for restriction digestions of the various PCR products are mentioned in Table 4. In parallel, the vectors of interest were digested with compatible enzymes (Table 5). A general map of the pETM-series vectors is provided in the Suppl. Figure 1, Appendices.

\begin{tabular}{llll}
\hline \hline Vector & Source & Tag & Restriction enzymes \\
\hline pETM-CoEx & EMBL (Heidelberg) & none & NcoI/Acc65I \\
pETM-11 & EMBL (Heidelberg) & N-terminal His & \\
pETM-ZZ & EMBL (Heidelberg) & N-terminal His 6 /ZZ double tag & NcoI/Acc65I \\
pET22b $(+)$ & Novagen (Darmstadt) & none & NdeI/BamHI \\
\hline \hline
\end{tabular}

Table 5. Description of the vectors used in the cloning procedure. The vector names, their sources, the encoded tag (when present) and the restriction enzymes employed for directed cloning purpose are mentioned. 
Digestions using appropriate restriction enzymes were performed as follows:

\begin{tabular}{|c|c|c|c|}
\hline \multicolumn{2}{|c|}{$\underline{40 \mu \mathrm{l} \text { PCR product double digestion reaction }}$} & \multicolumn{2}{|c|}{$\underline{100 \mu \mathrm{l} \text { vector double digestion reaction }}$} \\
\hline $4 \mu 1$ & 10X NEB buffer & $10 \mu 1$ & 10X NEB buffer \\
\hline $\mathrm{x} \mu \mathrm{l}$ & DNA $(2 \mu \mathrm{g})$ & $\mathrm{x} \mu \mathrm{l}$ & vector $(2 \mu \mathrm{g})$ \\
\hline $1 \mu 1$ & Enzyme $1(10 \mathrm{U} / \mu \mathrm{l})$ & $2 \mu 1$ & Enzyme $1(10 \mathrm{U} / \mu \mathrm{l})$ \\
\hline $1 \mu 1$ & Enzyme $2(10 \mathrm{U} / \mu \mathrm{l})$ & $2 \mu 1$ & Enzyme $2(10 \mathrm{U} / \mu \mathrm{l})$ \\
\hline $1 \mu 1$ & $\mathrm{BSA}(10 \mathrm{mg} / \mathrm{ml})$ & $2 \mu 1$ & BSA $(10 \mathrm{mg} / \mathrm{ml})$ \\
\hline у $\mu 1$ & $\mathrm{H}_{2} \mathrm{O}$ & у $\mu 1$ & $\mathrm{H}_{2} \mathrm{O}$ \\
\hline \multicolumn{2}{|c|}{ Incubation time: $4 \mathrm{~h}$} & \multicolumn{2}{|c|}{ Incubation time: $4 \mathrm{~h}$} \\
\hline \multicolumn{2}{|c|}{ Incubation temperature: according to NEB } & \multicolumn{2}{|c|}{ Incubation temperature: according to NEB } \\
\hline
\end{tabular}

\section{Ligation}

The digested PCR products and vectors were separated on agarose gel and purified using the QIAquick gel extraction kit (Quiagen, Hilden) according to the manufacturer's instructions. Subsequently, the purified PCR products were ligated to the linearized vectors. For optimal ligation efficiency, a molar ratio insert:vector of 3:1 was employed. Ligation reactions were performed as follows:

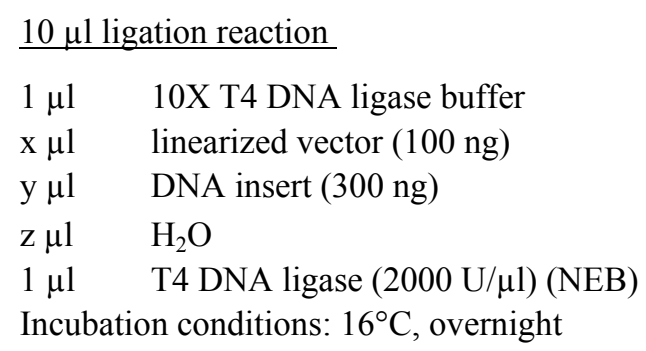

\section{E. Competent cells preparation by calcium chloride treatment}

The resulting plasmids were transformed in E. coli competent cells by heat shock method. These cells were prepared from an E. coli DH5 $\alpha$ strain (Invitrogen, USA) by calcium chloride treatment. Briefly, one colony of DH5 $\alpha$ strain was inoculated in $5 \mathrm{ml}$ Luria Bertani medium (LB, autoclaved prior usage at $121^{\circ} \mathrm{C}$ for $20 \mathrm{~min}$, containing $10 \mathrm{~g}$ tryptone, $5 \mathrm{~g}$ yeast extract, $10 \mathrm{~g} \mathrm{NaCl}$ and $\mathrm{ddH}_{2} \mathrm{O}$ up to 1 liter) and grown overnight at $37^{\circ} \mathrm{C}$. This pre-culture was inoculated in $50 \mathrm{ml} \mathrm{LB}$ medium. Cells were grown to mid-log phase $\left(\mathrm{OD}_{595}\right.$ of 0.7$)$. Next, they were harvested for $10 \mathrm{~min}$ at $2000 \mathrm{rpm}$ and resuspended in $25 \mathrm{ml}$ of ice-cold $50 \mathrm{mM} \mathrm{CaCl}_{2}$. After centrifugation for $10 \mathrm{~min}$ at $2000 \mathrm{rpm}$, cells were treated with $3 \mathrm{ml}$ of ice-cold $50 \mathrm{mM} \mathrm{CaCl}_{2}$ supplemented with $10 \%$ glycerol, aliquoted $(100 \mu 1)$ and shock-frozen in liquid nitrogen. 


\section{F. Transformation of $E$. coli cells by heat shock}

Prior to transformation, a $100 \mu 1$ aliquot of competent cells was thawed on ice. The ligation products were then added to competent cells. Next, this mixture was incubated on ice for $30 \mathrm{~min}$, heat shocked at $42^{\circ} \mathrm{C}$ for $30 \mathrm{~s}$ and then retransferred for $2 \mathrm{~min}$ on ice. 900 $\mu \mathrm{l}$ of LB medium were added to the cells and the mixture was incubated at $37^{\circ} \mathrm{C}$ for $1 \mathrm{~h}$ with gentle shaking. Finally, cells were spread onto LB plates (6 g agar in $400 \mathrm{ml}$ LB broth, autoclaved at $121^{\circ} \mathrm{C}$ for $20 \mathrm{~min}$, and subsequently supplemented to the appropriate antibiotic for selection) and incubated at $37^{\circ} \mathrm{C}$ overnight.

\section{G. Mini-preparation of plasmid DNA}

From the resulting colonies, plasmid DNA was extracted using the QIAprep spin miniprep kit (Qiagen), according to the instructions of the manufacturer. Positive clones were identified by restriction mapping.

\section{H. DNA sequencing}

The sequence accuracy of PCR products for each construct was confirmed by automated Sanger dideoxynucleotide sequencing (Sanger et al. 1977). The reactions and PCR cycling programs were established as follows:

$\begin{array}{ll}20 \mu \mathrm{l} \text { sequencing reaction } \\ 3.0 \mu \mathrm{l} & \text { sample (300 ng) } \\ 1.0 \mu \mathrm{l} & \text { sequencing primer }(10 \mathrm{pmol} / \mu \mathrm{l}) \\ 10.0 \mu \mathrm{l} & \mathrm{H}_{2} \mathrm{O} \\ 6.0 \mu \mathrm{l} & \text { BigDye }\end{array}$

Following the temperature cycling, reactions were spun down briefly. To precipitate the samples, $15 \mu 13 \mathrm{M}$ sodium acetate $\mathrm{pH}$ 5.3, $65 \mu 1 \mathrm{H}_{2} \mathrm{O}$ and $300 \mu 1100 \%$ ethanol were added and mixed. The samples were centrifuged at 13,000 rpm for $20 \mathrm{~min}$ at $15^{\circ} \mathrm{C}$. The pellets were washed once with $750 \mu 170 \%$ ethanol, air-dried and resuspended in $25 \mu \mathrm{l}$ of template suppression reagent. The DNA was sequenced on an ABI PRISM 310 Genetic Analyzer (Applied Biosystems, Weiterstadt) by M. Killian or G. Dowe, MPI for Biophysical Chemistry, Göttingen.

\begin{tabular}{|c|c|c|}
\hline \multicolumn{3}{|c|}{$\underline{\text { PCR program }}$} \\
\hline $1 x$ & $96^{\circ} \mathrm{C}$ & $1 \mathrm{~min}$ \\
\hline $25 \mathrm{x}$ & $96^{\circ} \mathrm{C}$ & $30 \mathrm{~s}$ \\
\hline & $55^{\circ} \mathrm{C}$ & $30 \mathrm{~s}$ \\
\hline & $60^{\circ} \mathrm{C}$ & $4 \mathrm{~min}$ \\
\hline
\end{tabular}

hold temperature at $4^{\circ} \mathrm{C}$ 
The sequences obtained were verified by comparison with sequences published in the public database using the Vector NTI program (http://www.invitrogen.com/content.cfm?pageid=10373) and Blast 2 sequences program (http://www.ncbi.nlm.nih.gov/blast/bl2seq/wblast2.cgi).

\section{Site-directed mutagenesis}

Several deletion constructs were produced using the QuickChange Site-Directed Mutagenesis Kit (Stratagene, Heidelberg) (Table 6). The resulting truncated constructs were verified by sequencing.

\begin{tabular}{|c|c|c|}
\hline Gene product & Sequence $\left(5^{\prime} \rightarrow 3^{\prime}\right) /$ Mutagenized codon & Vector \\
\hline \multirow[t]{2}{*}{ tmaL12 NTD } & F: CTCGAAGACAAATTTGGATAGACTGCTGCTGCACCTGTG / 31 & $\mathrm{pETM} 22 \mathrm{~b}(+)$ \\
\hline & R: CACAGGTGCAGCAGCAGTCTATCCAAATTTGTCTTCGAG & \\
\hline \multirow[t]{2}{*}{ tmaL12 NTD/hinge } & F: GCTGCCGGTGCCGCTCAGTAAGAAAAGACAGAGTTTGAC / 54 & $\mathrm{pETM} 22 \mathrm{~b}(+)$ \\
\hline & R: GTCAAACTCTGTCTTTTCTTACTGAGCGGCACCGGCAGC & \\
\hline \multirow[t]{2}{*}{$\operatorname{tma} \mathrm{L} 10_{\triangle 2 \mathrm{DBS}}$} & F: GTGTGAAAGCTCCGATTACCTAGCTTGTGTTTGCATTGAGTGG / 154 & pETM-ZZ \\
\hline & R: CCACTCAATGCAAACACAAGCTAGGTAATCGGAGCTTTCACAC & \\
\hline \multirow[t]{2}{*}{$\operatorname{tma} \mathrm{L} 10_{\triangle 1 \mathrm{DBS}}$} & F: GTTTGCATTGAGTGGTATTTTGTAGAATCTCGTGTATGTGCTCAATG / 164 & pETM-ZZ \\
\hline & R: CATTGAGCACATACACGAGATTCTACAAAATACCACTCAATCCAAAC & \\
\hline
\end{tabular}

Table 6. Oligonucleotide primers used for mutagenesis. The gene product names, primers used, STOP codon inserted within the primers (in red) and vectors used are indicated. In each primer pair, the forward primer is denominated $\mathrm{F}$ and the reverse $\mathrm{R}$, respectively. The mutagenesis of tmaL10:L12 NTD and tmaL10:L12 NTD/hinge were performed using a previously described plasmid (Wahl et al. 2000b).

\section{Protein production}

\section{A. Expression of native proteins}

- aaeL10, aaeL12, tmaL12 CTD, tmaEF-Tu(Gd) (using pETM-11 for the latter two) were individually expressed in Rosetta(DE3) E. coli cell strain.

- ecoL10:L12 complex (from a biscistronic, pGEX-5x-3-based plasmid with sequential genes for GST-L10 and L12; this plasmid was kindly offered by M.V. Rodnina, Witten) was expressed in Rosetta(DE3) cell strain.

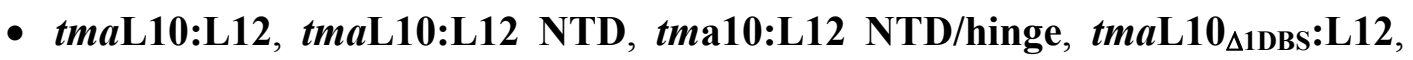

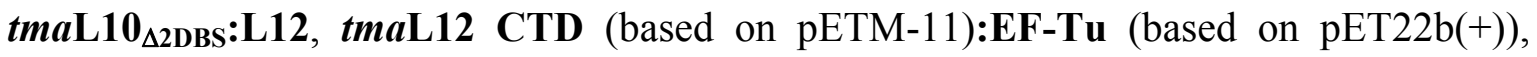
tmaL12 CTD (based on pET22b(+)):EF-Tu (based on pETM-11), tmaL10 (based on the pETM-ZZ):ecoL12 (based on pT7-6::rplL (Oleinikov et al. 1993)) were co-expressed after 
co-transformation in Rosetta(DE3). For co-transformation, $1 \mu 1(0.5 \mu \mathrm{g} / \mu \mathrm{l})$ of each plasmid was introduced in $100 \mu 1$ Rosetta(DE3) cells, by heat-shock transformation procedure.

- tmaL11 was expressed in BL12(DE3) cell strain.

For expression purpose, E. coli cells from Rosetta(DE3) (Novagen, Darmstadt) and BL21(DE3) (Novagen) strains were prepared by calcium chloride treatment.

Following transformation, the cells were used to inoculate a starter culture of 100 $\mathrm{ml}$ LB supplemented with the appropriate antibiotics for selection. The antibiotics used in this study had the following concentrations: $30 \mu \mathrm{g} / \mathrm{ml}$ kanamycin (Boehringer, Mannheim), $34 \mu \mathrm{g} / \mathrm{ml}$ chloramphenicol (Boehringer) and $100 \mu \mathrm{g} / \mathrm{ml}$ ampicillin (Sigma, Deisenhofen). The starter culture was grown overnight at $37^{\circ} \mathrm{C}$. Next, the cells were harvested, resuspended in $12 \mathrm{ml}$ fresh LB medium and distributed among $61 \mathrm{LB}$ medium supplemented with the required antibiotics. Cells were grown in 11 shaking cultures at $37^{\circ} \mathrm{C}$ and $200-250 \mathrm{rpm}$ to mid-log phase $\left(\mathrm{OD}_{595}\right.$ of $\left.0.7-0.8\right)$, induced by addition of $1 \mathrm{mM}$ isopropyl- $\beta$-D-thiogalactopyranoside (Sigma) and harvested $4 \mathrm{~h}$ after induction. To avoid protease degradation of the overexpressed proteins, two Complete EDTA-free Protease Inhibitor Cocktail tablets (Roche, Mannheim) were added.

\section{B. Purification of native proteins}

\section{Purification of the aaeL10:L12 complex}

A crude cell extract was prepared by sonification on ice with a Branson (Danbury, USA) macrotip (50\% pulsed, output 7) in lysis buffer ( $50 \mathrm{mM}$ Tris- $\mathrm{HCl}, 50 \mathrm{mM} \mathrm{NaCl}, 5$ mM DTT, 2 mM EDTA, pH 7.0, supplemented with lysozyme (Sigma)) and centrifuged for $45 \mathrm{~min}$ at $30,000 \mathrm{x} \mathrm{g}$ in a SA-600 rotor, run on a Sorvall centrifuge (Kendro, USA). The resulting S-100 fractions containing L10 and L12 overexpressed proteins were combined to reconstitute the aaeL10:L12 complex. To ensure complex formation, combined cell lysates were incubated for $30 \mathrm{~min}$ at room temperature. Next, the complex was further purified by heat treatment at $90^{\circ} \mathrm{C}$. This purification step was rendered possible by the thermophilic properties of Aquifex aeolicus bacterium. Thus, most of E. coli proteins present in the cell lysate were denaturated and precipitated through a centrifugation step at $30,000 \mathrm{x}$ g for $20 \mathrm{~min}$. The supernatant was loaded onto a HiTrap DEAE FF anion exchange column (Amersham Biosciences), which had been previously equilibrated with buffer A (50 mM Tris, $2 \mathrm{mM}$ DTT, pH 7.0). The aaeL10:L12 complex 
was eluted with a linear gradient of buffer A and B (50 mM Tris, $500 \mathrm{mM} \mathrm{LiCl}$ or NaCl, 2 mM DTT, pH 7.0). As identified on a 15\% SDS-PAGE (Laemmli 1970), the fractions containing the aaeL10:L12 complex were pooled, concentrated to $2 \mathrm{ml}$ (Millipore, Schwalbach) and loaded onto a HiLoad Superdex 75 (26/60) prep grade gel filtration column (Amersham Biosciences) run with Buffer C (10 mM, $50 \mathrm{mM} \mathrm{LiCl}$ or NaCl, $2 \mathrm{mM}$ DTT, $\mathrm{pH}$ 7.0) to remove the uncomplexed protein and allow transfer to a buffer appropriate for crystallization. Thus, the fractions containing the complex were pooled, concentrated to $20 \mathrm{mg} / \mathrm{ml}$ and frozen in liquid nitrogen ( $\mathrm{KGV}$, Karlsruhe). The protein complex concentration was first measured by Bradford assay (Bradford 1976) and subsequently estimated using in-gel comparisons with proteins of known concentrations and according to (Wahl et al. 2000b). The authenticity of the purified complex was confirmed by mass spectrometry (performed by M. Raabe, U. Pleßmann, H. Urlaub, Research Group Mass Spectrometry, MPI for Biophysical Chemistry, Göttingen).

\section{Purification of tmaL10:L12, tmaL10:L12 NTD, tmaL10:L12 NTD/hinge, tmaL10:ecoL12, tmaL10 ${ }_{\Delta 2 \mathrm{DBS}}$, tmaL12 CTD, tmaEF-Tu(Gd) complexes}

Unless otherwise specified, all purification steps were performed at $4^{\circ} \mathrm{C}$. The pellets obtained from $6 \times 11$ culture were resuspended in $10 \mathrm{ml}$ lysis buffer $(20 \mathrm{mM}$ Tris$\mathrm{HCl}, 10 \mathrm{mM}$ imidazole, $150 \mathrm{mM} \mathrm{NaCl}$, 0.2\% Igepal CA-630, $2 \mathrm{mM} \beta$-mercaptoethanol, pH 8.0, supplemented with Pefabloc SC (Biomol, Hamburg)). A crude cell extract was prepared by sonification. The cell lysate was cleared for $45 \mathrm{~min}$ at $30,000 \mathrm{x} \mathrm{g}$. The resulting S-100 fraction was purified via affinity chromatography on Ni-NTA column (Quiagen). The proteins of interest bound to the Ni-NTA beads via their His 6 -tags. The bound proteins were then washed with $\mathrm{W} 1$ (20 mM Tris, $10 \mathrm{mM}$ imidazole, $150 \mathrm{mM}$ $\mathrm{NaCl}$, pH 8.0), W2 (20 mM Tris, $10 \mathrm{mM}$ imidazole, $1 \mathrm{M} \mathrm{NaCl}, \mathrm{pH} 8.0$ ) and $\mathrm{W} 3$ (20 mM Tris, $30 \mathrm{mM}$ imidazole, $150 \mathrm{mM} \mathrm{NaCl}, \mathrm{pH} \mathrm{8.0)}$ buffers, to remove the E. coli proteins non-specifically attached to the beads. Next, the proteins were eluted with buffer A (20 $\mathrm{mM}$ Tris, $150 \mathrm{mM} \mathrm{NaCl}, 300 \mathrm{mM}$ imidazole, $\mathrm{pH} \mathrm{8.0)}$ and brought into low-salt buffer $\mathrm{B}$ (20 mM Tris-HCl, 100 mM NaCl, pH 8.0) using PD-10 columns (Amersham Biosciences). The tags were removed by cleavage with tobacco etch virus protease $(1 \mathrm{ml}$ TEV protease $(0,5 \mathrm{mg} / \mathrm{ml})$ was incubated with the proteins resulting from 11 culture for $2 \mathrm{~h}$ at RT and subsequently overnight $4^{\circ} \mathrm{C}$ ) and the samples were re-purified on Ni-NTA beads to remove the TEV protease and the $\mathrm{His}_{6}$ or $\mathrm{His}_{6} / \mathrm{ZZ}$ tags. The flow-through was heated at $80^{\circ} \mathrm{C}$ for 
$20 \mathrm{~min}$ and centrifuged (30 $\mathrm{min}, 10,000 \mathrm{xg}$ ). This purification step was possible due to the thermophilic properties of this bacterium. Thus, most of the E. coli proteins present in the cell lysate were denaturated and precipitated in a centrifugation step at 30,000 x g for 20 min. (The purification via heat denaturation was omitted for tmaL10:ecoL12, as the ecoL12 protein is not stable above $65^{\circ} \mathrm{C}$ ). The supernatant was concentrated and further purified by size exclusion chromatography (Superdex 75) with buffer C (10 mM Tris-HCl, $50 \mathrm{mM} \mathrm{NaCl}, 2 \mathrm{mM}$ DTT, $\mathrm{pH} \mathrm{8.0)}$. Peak fractions corresponding to purified proteins or protein complexes were identified on SDS polyacrylamide gels (15 - 18\%), pooled, concentrated to $8-10 \mathrm{mg} / \mathrm{ml}$, frozen in liquid nitrogen and stored at $-80^{\circ} \mathrm{C}$. The purity of the resulting preparations was estimated at $>90 \%$ according to Coomassie blue-stained SDS gels. The authenticity of the purified products was confirmed by mass spectrometry.

\section{Purification of the ecoL10:L12 complex}

The ecoL10:L12 complex was isolated from a clarified lysate on glutathione affinity beads (Amersham Biosciences). Following several washing steps with $500 \mathrm{mM}$ Tris $\mathrm{pH}$ 8.0, the complex was eluted with $10 \mathrm{mM}$ reduced glutathione, $500 \mathrm{mM}$ Tris $\mathrm{pH}$ 8.0. Subsequently, the protein complex was buffer exchanged in a low salt buffer $50 \mathrm{mM}$ Tris, $100 \mathrm{mM} \mathrm{NaCl}, 5 \mathrm{mM} \mathrm{CaCl}_{2}$, pH 8.0 (recommended for Factor Xa cleavage) with PD10 columns. The GST tag was next removed by cleavage with $5 \mu$ Factor Xa $(2 \mathrm{U} / \mu 1$; Novagen) for $20 \mathrm{~h}$ at $4^{\circ} \mathrm{C}$ and $3 \mathrm{~h}$ at $\mathrm{RT}$ on a head-over-tail rotor. This reaction was stopped by adjunction of $0.5 \mathrm{mM}$ PMSF (Boehringer) for $1 \mathrm{~h}$. The protein complex was further purified on a heparin column (HiTrap Heparin $5 \mathrm{ml}$; Amersham Biosciences) with a linear gradient of buffer A (20 mM Tris, $50 \mathrm{mM} \mathrm{NaCl}, 2 \mathrm{mM}$ DTT pH 8.0) to buffer B (20 mM Tris, $500 \mathrm{mM} \mathrm{NaCl}, 2 \mathrm{mM}$ DTT pH 8.0) and on a Superdex 75 size exclusion column (Amersham Biosciences) operated with $10 \mathrm{mM}$ Tris, $50 \mathrm{mM} \mathrm{NaCl}, 2 \mathrm{mM}$ DTT, $\mathrm{pH}$ 8.0. The fractions containing the ecoL10:L12 complex were further concentrated (Millipore) to $10 \mathrm{mg} / \mathrm{ml}$, frozen in liquid nitrogen and stored at $-80^{\circ} \mathrm{C}$.

\section{Purification of the tmaL11 protein}

The tmaL11 protein was isolated from a clarified lysate on Ni-NTA affinity beads, washed and eluted as described in section II.B.2. Next, the protein was transferred in a low salt buffer containing $20 \mathrm{mM}$ MES, $150 \mathrm{mM} \mathrm{NaCl}$, pH 6.5 using PD-10 columns. Following the removal of the $\mathrm{His}_{6}$-tag by TEV protease, the protein was heated at $80^{\circ} \mathrm{C}$ for 
$20 \mathrm{~min}$ and centrifuged for $20 \mathrm{~min}$ at $10,000 \mathrm{x}$ g. The supernatant was re-purified on NiNTA beads and subsequently buffer exchanged in $50 \mathrm{mM}$ MES pH 6.5 with a HiTrap desalting column (Amersham Biosciences), concentrated to $1 \mathrm{ml}$ and loaded onto a HiTrap $\mathrm{CM}$ cation exchange column. The protein was eluted with a linear gradient of buffer A (50 mM MES, 2 mM DTT, pH 6.5) and B (50 mM MES, $1 \mathrm{M} \mathrm{NaCl,} 2$ mM DTT, pH 6.5). The peak fractions containing the purified protein were combined and buffer exchanged with PD-10 columns in buffer $\mathrm{C}(10 \mathrm{mM}$ Tris, $50 \mathrm{mM} \mathrm{NaCl}, 2 \mathrm{mM}$ DTT $\mathrm{pH}$ 7.0) for crystallization and in buffer D $\left(20 \mathrm{mM} \mathrm{NaH}_{2} \mathrm{PO}_{4}, \mathrm{Na}_{2} \mathrm{HPO}_{4}, 150 \mathrm{mM} \mathrm{NaCl}, 1 \mathrm{mM}\right.$ DTT, $\mathrm{pH}$ 7.0) for calorimetric studies.

\section{Purification of TEV protease}

This protein was expressed as a His ${ }_{6}$-tagged protein in BL12(DE3)pLysS cells. A glycerol stock containing cells expressing TEV (Invitrogen) was used to inoculate $100 \mathrm{ml}$ LB medium supplemented with $30 \mu \mathrm{g} / \mathrm{ml}$ kanamycin and $34 \mu \mathrm{g} / \mathrm{ml}$ chloramphenicol. The cells were grown overnight at $37^{\circ} \mathrm{C}$. This pre-culture was used to inoculate 21 of $\mathrm{LB}$ medium containing $30 \mu \mathrm{g} / \mathrm{ml}$ kanamycin. These cells were grown to mid-log phase. Prior induction, they were cooled at $25^{\circ} \mathrm{C}$, induced by adjunction of $1 \mathrm{mM} \mathrm{IPTG}$, grown at $25^{\circ} \mathrm{C}$ and $200 \mathrm{rpm}$ and harvested $3 \mathrm{~h}$ post induction. The resulting pellet was resuspended in lysis buffer (20 mM Tris-HCl, $10 \mathrm{mM}$ imidazole, $150 \mathrm{mM} \mathrm{NaCl}$, 0.2\% Igepal CA-630, 2 $\mathrm{mM} \beta$-mercaptoethanol, $\mathrm{pH} 8.0$ ). A crude cell extract from a 21 of culture was prepared by sonification in lysis buffer and subsequently centrifuged at $30,000 \mathrm{x}$ g for $45 \mathrm{~min}$. The lysate was passed three times over a Ni-NTA column, washed with W1, W2, W3 (described in section II.B.2.) and eluted with $300 \mathrm{mM}$ imidazole, $2 \mathrm{mM} \beta$ mercaptoethanol, $20 \%$ glycerol. Next, the protein was buffer exchanged in $20 \mathrm{mM}$ Tris, $100 \mathrm{mM} \mathrm{NaCl}, 20 \%$ glycerol, 2 mM DTT, pH 8.0 using PD-10 columns. Subsequently, one volume of glycerol was added. The protease was then rapidly aliquoted to prevent degradation, flash-frozen in liquid nitrogen and stored at $-80^{\circ} \mathrm{C}$.

\section{Expression of the selenomethionine-derivatized protein}

The expression of the selenomethionine-containing tmaL10:L12 NTD complex was performed using the methionine auxotrophic E. coli B834(DE3)pLysS (Novagen) strain according to (Budisa et al. 1995). 
Following co-transformation with plasmids containing tmaL10 and tmaL12 NTD genes, cells were added to a starter culture of $100 \mathrm{ml}$ minimal medium supplemented with the required antibiotics (30 $\mu \mathrm{g} / \mathrm{ml}$ kanamycin, $34 \mu \mathrm{g} / \mathrm{ml}$ chloramphenicol and $100 \mu \mathrm{g} / \mathrm{ml}$ ampicillin) and grown overnight at $37^{\circ} \mathrm{C}$. The minimal medium contained a salt solution (comprising $\mathrm{NaCl},\left(\mathrm{NH}_{4}\right)_{2} \mathrm{SO}_{4}, \mathrm{MgSO}_{4}, \mathrm{CaSO}_{4} \times 2 \mathrm{H}_{2} \mathrm{O}, \mathrm{FeH}_{8} \mathrm{~N}_{2} \mathrm{O}_{8} \mathrm{~S}_{2} \times 6 \mathrm{H}_{2} \mathrm{O}$ supplemented with $0.4 \%$ glucose, $10 \mathrm{mg} / \mathrm{ml}$ thiamine and $10 \mathrm{mg} / \mathrm{ml}$ biotin), a trace elements solution (containing $1 \mu \mathrm{g} / \mathrm{ml}$ of each $\mathrm{MnCl}_{2}, \mathrm{CuSO}_{4} \times 5 \mathrm{H}_{2} \mathrm{O}, \mathrm{Na}_{2} \mathrm{MoO}_{4}, \mathrm{ZnSO}_{4}$ compound) and a potassium phosphate solution containing $0.083 \mathrm{~g} / 1$ of all amino acids except methionine. To the final composition of the minimal medium $0.3 \mathrm{mM}$ selenomethionine was added. The overnight culture was harvested, resuspended into $12 \mathrm{ml}$ of fresh minimal medium and subsequently distributed among 61 culture. The cells were grown at $37^{\circ} \mathrm{C}, 250 \mathrm{rpm}$ to mid$\log$ phase, induced with $1 \mathrm{mM}$ IPTG and harvested $8 \mathrm{~h}$ after induction.

\section{Purification of the selenomethionine-derivatized protein}

The protocol of purification used was as described for the native tmaL10:L12 NTD protein complex (section II.B.2.). The final preparation was concentrated to $8 \mathrm{mg} / \mathrm{ml}$.

\section{Crystallization}

\section{A. Principles of protein crystallography}

The process of X-Ray crystallographic structure determination of a protein consists of: (i) crystal growth, (ii) data collection, (iii) solution of the phase problem, (iv) generation of the atomic model (model building), and (v) fitting the atomic model to the measured data (refinement) (Drenth 1994; Rodes 2000).

\section{Crystal growth}

The first essential step in determining the X-ray structure of a protein is to grow crystals to sufficient size and quality. A highly pure and concentrated protein $(5-20$ $\mathrm{mg} / \mathrm{ml}$ ) is dissolved in a suitable solvent from which it must be precipitated in a crystalline form. Crystallization occurs when the concentration of the protein in solution is greater than its limit of solubility (protein supersaturation). There are three stages of crystallization: nucleation, growth and cessation of growth. 
Crystallization methods include: vapor diffusion, dialysis, microbatch, seeding etc. Among these methods, one of the most commonly used is vapor diffusion. In this approach, the initial reagent concentration in the droplet is lower than that in the reservoir. Over time, the reservoir will pull water from the droplet in a vapor phase, such that an equilibrium will occur between the drop and the reservoir. During this equilibration process, the sample is also concentrated, thereby increasing the supersaturation of the sample in the drop. Two vapor diffusion techniques are frequently employed: sitting drop and hanging drop. In the sitting drop technique (frequently employed in the present work), one places a small droplet of the sample mixed with crystallization reagent on a platform in vapor equilibration with the reagent (Figure 7). In the hanging drop technique, one places a small droplet of the sample mixed with the crystallization reagent on a siliconized glass cover slide inverted over the reservoir in vapor equilibration with the reagent.

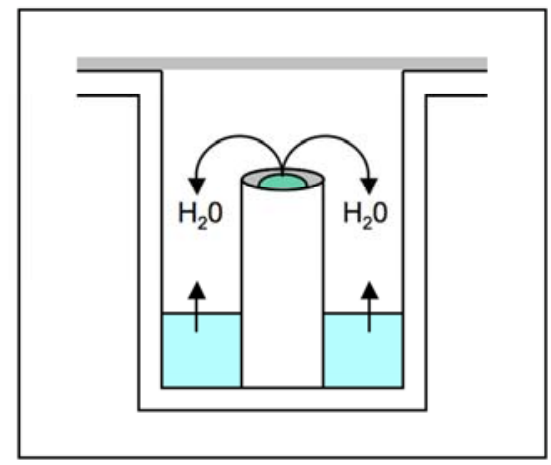

Figure 7. Sitting drop vapor diffusion technique. A drop composed of a mixture of sample and reservoir solution is placed in vapor equilibration with a liquid reservoir of reagent. In order to achieve equilibrium, the water leaves the droplet and eventually ends up in the reservoir. If the reservoir solution contains a volatile compound (e.g. alchool), the equilibrium will be reached through an interchange between both droplet and reservoir solutions.

\section{Data collection}

The resulting crystal is exposed to X-rays for structure determination, as the wavelength of X-rays is comparable to the interatomic distances of a crystal. The conventional X-ray sources are: (i) the sealed tube (in which X-rays are produced by bombarding a metal target, usually copper, with electrons produced by a heated filament and accelerated by an electric filed), (ii) the rotating anode, with a higher intensity, (iii) the synchrotron with a high intensity $\mathrm{X}$-ray radiation and high tunability (allowing the selection of radiation with a wavelength of $1 \AA$ or below). When a crystal is placed in the path of an X-ray beam, it diffracts the source beam into many discrete beams. The X-rays 
are scattered exclusively by the electrons in the atoms. Each of the resulting beams will produce a distinct spot (reflection) on a detector. To collect a full set of reflections, it is necessary to rotate the crystal, in small steps, by an angle determined by its degree of symmetry. Each reflection is given co-ordinates $h, k, l$ (reciprocal space coordinates) and an intensity. Because X-rays cannot be optically focused (they simply continue in a straight line when they enter most solids), each ray that caused a diffraction spot has to be traced back to the structure that diffracted it. To achieve this tracing, one needs to determine not only the position and intensity of each spot, but also the phase. Each diffracted ray from a crystal unit cell recorded by a reflection $h k l$ is described by a structure factor $F(h k l)$. The latter represents a vector characterized by frequency (that of the X-ray source), amplitude $|F(h k l)|$ (derived from the intensities of the spots) and phase $\alpha(h k l)$. Following the diffraction pattern acquisition, an electron density map can be calculated, which is basically the image of the structure of interest. The calculation of the electron density $\rho$ at every position $\mathrm{x}, \mathrm{y}, \mathrm{z}$ (real space coordinates) in the unit cell is done with a Fourier transform and is defined as:

$$
\rho(\mathrm{xyz})=\frac{1}{V} \sum_{h} \sum_{k} \sum_{l}|F(h k l)| \exp [-2 \pi i(h \mathrm{x}+k \mathrm{y}+l \mathrm{z})+i \alpha(h k l)]
$$

where $V$ is the unit cell volume and $i$ represents the contribution of each atom.

Thus, to obtain the electron density throughout the unit cell, one needs to know the amplitude $|F(h k l)|$ and the relative phase angles $\alpha(h k l)$. The amplitude results from the diffraction data, as it is the square root of the measured intensity $I(h k l)$. However, the phase angles cannot be derived from the diffraction pattern.

\section{Solution of the phase problem}

Several methods have been developed in order to deduce phases for reflections, including Molecular Replacement (MR), Single Isomorphous Replacement (SIR), Multiple Isomorphous Replacement (MIR), Single-wavelength Anomalous Dispersion (SAD) and Multi-wavelength Anomalous Dispersion (MAD). Combinations of several of the above mentioned approaches, e.g. MIRAS (Multiple Isomorphous Replacement using Anomalous Scattering) and SIRAS (Single Isomorphous Replacement using Anomalous Scattering) proved to be useful for experimental phasing of a number of structures. Three of the most common methods to obtain phases are MIR, MAD and MR. 
Multiple isomorphous replacement (MIR) represents a method of choice for determination of phases of a completely unknown structure. This method involves collection of data from crystals of the protein alone, and crystals soaked in various heavyatom compounds (e.g. ions or ionic complexes of $\mathrm{Hg}, \mathrm{Pt}, \mathrm{Au}, \mathrm{Ta}, \mathrm{U}$ etc.). If heavy atoms bind specifically to the protein, their locations can be identified, and the phase problem can be solved from the difference in the structure factors between the protein and its heavyatom derivatives (isomorphous differences). Frequently, more than one heavy atom derivative is necessary due to the ambiguity of the phase angle. In addition, heavy atoms absorb X-rays of a specified wavelength. As a result of this absorption, the Friedel law postulating that $|F(h k l)|=|F(-h-k-l)|$ and $\alpha(h k l)=-\alpha(-h-k-l)$ does not hold. The inequality of symmetry-related reflections is called anomalous dispersion. The measurement of the differences between Friedel pairs (termed Bijvoet differences) leads to an additional source of information from the heavy atom. Thus, MIR can make use of both isomorphous and Bijvoet differences.

Multi-wavelength anomalous dispersion (MAD) can be considered an ideal case of MIR because (i) scattering of preexisting atoms in the crystal is varied by changing the $\mathrm{X}$ ray wavelength and (ii) only one crystal is measured, resulting in a perfect isomorphism. An example of an atom, which can be scattered by changing the X-ray wavelength, is selenium (this atom is introduced during the preparation of protein, by replacing the conventional methionine with selenomethionine). In this approach the wavelength is varied around the absorption edge of such an atom. At these wavelengths, there is significant variation in the real f' and imaginary components f', of the anomalous scattering of these special atoms. The best is to select a peak wavelength $(\lambda 1)$ where $f^{\prime \prime}$ has its maximum, inflection point $(\lambda 2)$ where $f^{\prime}$ has it maximum and one or more remote points at which $\mathrm{f}^{\prime}$ is substantially closer to zero than at the edge. However, the signal obtained from anomalous scattering is normally quite small (compared to conventional heavy atom phasing), therefore high occurrence of scatterers (a rule of thumb is one Se per 15-20 kD) and precise measurement of the signals are required.

Molecular replacement (MR) can be used when a good model for a reasonable portion of the structure in the crystal is known. As a rule of thumb, MR is straightforward if the known model and the unknown protein share approximately $40 \%$ sequence identity or if, for another reason, the two structures are expected to have a very similar fold of their polypeptide chain. Placement of the molecule in the target unit cell requires a rotation and 
a translation step. In the rotation step, the spatial orientation of the known and the unknown molecule with respect to each other is determined, while in the next step, the translation needed to superimpose the now correctly oriented molecule onto the other molecule is calculated.

\section{Model building}

An interpretable electron density map can usually be produced after density modification and phase combination. Following these steps, the atoms can be traced in the electron density.

\section{Structure refinement}

Refinement is the process of adjusting the model to fit to a closer agreement between the calculated and the observed structure factors and is represented by the crystallographic R-factor. In addition to lowering the R-factor, the structural parameters should indicate a model that is chemically, stereochemically and conformationally reasonable.

\section{B. Crystallization experiments}

Crystallization was performed on the in-house high-throughput facility, a nano drop robot (Cartesian Dispensing System MicroSys 4000XL, Genomic Solutions Ltd, UK). This apparatus was programmed to set up 100 nl-scale vapor diffusion sitting drop crystallization experiments in 96-well plates (containing $100 \mu 1$ reservoir solutions). The process was controlled by a computer using AxSys software. An overview of the crystallization experiments performed with different protein complexes is provided in Table 7. The initial conditions that yielded crystals using the nano drop robot were subsequently scaled up to microliter range and refined by screening the effects of precipitant, additives and $\mathrm{pH}$. Thus, droplets were set up by mixing $0.5-1 \mu 1$ protein $(8-$ $20 \mathrm{mg} / \mathrm{ml}$ ) with $1 \mu \mathrm{l}$ reservoir and were equilibrated against $500 \mu \mathrm{l}$ reservoir. The techniques used for the refinement of the crystallization conditions were: sitting drop (for most of the screens) and hanging drop vapor diffusion. 


\begin{tabular}{|c|c|c|c|c|}
\hline Proteins & $\begin{array}{c}\text { Conditions } \\
\text { screened }\end{array}$ & Source & $\mathrm{T}^{\circ} \mathrm{C}$ & $\begin{array}{c}\text { Crystal } \\
\text { forms }\end{array}$ \\
\hline aaeL10:L12 & 288 & $\begin{array}{c}\text { Hampton Research (USA) } \\
\text { Emerald BioStructures (USA) } \\
\text { Sodium malonate screen (made) } \\
\text { Ammonium sulfate screen (made) }\end{array}$ & 4,20 & \\
\hline tmaL10:L12 & 1344 & $\begin{array}{c}\text { Hampton Research } \\
\text { Emerald BioStructures } \\
\text { Sodium malonate screen } \\
\text { Ammonium sulfate screen } \\
\text { Nextal Biotechnologies (Canada) }\end{array}$ & 4,20 & 1 \\
\hline tmaL10:L12 NTD & 384 & $\begin{array}{c}\text { Hampton Research } \\
\text { Emerald BioStructures } \\
\text { Nextal Biotechnologies }\end{array}$ & 20 & 3 \\
\hline tmaL10 ${ }_{\Delta 2 \mathrm{DBS}}: \mathrm{L} 12$ & 288 & $\begin{array}{c}\text { Hampton Research } \\
\text { Nextal Biotechnologies }\end{array}$ & 20 & \\
\hline tmaL12 CTD:EF-Tu(Gd):GMPPNP & 672 & $\begin{array}{c}\text { Hampton Research } \\
\text { Nextal Biotechnologies }\end{array}$ & 20 & 1 \\
\hline tmaL12 CTD:EF-Tu(Gd):GDP & 672 & $\begin{array}{c}\text { Hampton Research } \\
\text { Nextal Biotechnologies }\end{array}$ & 20 & \\
\hline tmaL12 CTD:EF-G:GMPPNP & 672 & $\begin{array}{c}\text { Hampton Research } \\
\text { Nextal Biotechnologies }\end{array}$ & 20 & \\
\hline tmaL12 CTD:L11 & 1152 & $\begin{array}{c}\text { Hampton Research } \\
\text { Nextal Biotechnologies }\end{array}$ & 20 & \\
\hline
\end{tabular}

Table 7. High-throughput crystallization experiments with several protein complexes from Thermotoga maritima and Aquifex aeolicus.

- tmaL10:L12 yielded needle-like crystals after 7 days at $20^{\circ} \mathrm{C}$ in several conditions containing PEG 3350 as a precipitant. After improvement, two conditions gave rise to single, large, needle-shaped crystals. The optimized reservoir formulations were:

- condition I (derived from Index screen condition 72, Hampton Research): $0.2 \mathrm{M} \mathrm{NaCl}$, $0.1 \mathrm{M}$ Tris $\mathrm{pH}$ 8.0, 20\% PEG 3350 (also with $\mathrm{pH}$ ranging from 7.0 to 8.0);

- conditions II (derived from Index screen condition 80, Hampton Research): $0.4 \mathrm{M}$ ammonium acetate, $\mathrm{pH} 7.2$, 25\% PEG 3350 (similar crystals were grown in the same conditions with $\mathrm{pH}$ ranging from 7.2-8.2).

tmaL10:L12 crystals obtained from condition I and II could be directly frozen in a liquid nitrogen stream and subjected to diffraction data acquisition.

- Crystals of tmaL10:L12 NTD appeared within a day at $20^{\circ} \mathrm{C}$. Three crystal forms were obtained from the following conditions: 
- crystal Form I (SeMet): 0.2 M ammonium acetate, 0.1 M Na-HEPES, pH 7.2, 42\% MPD (from Index screen number 52, Hampton Research);

- crystal Form II (native): $0.2 \mathrm{M} \mathrm{MgCl}_{2}, 0.1 \mathrm{M}$ imidazole, pH 8.0, 40\% MPD (condition number 34 of Cryo I screen, Emerald BioStructures);

- crystal Form III (native): Na-acetate, pH 4.5, 50\% ethylene glycol, 5 \% PEG 1000 (final pH 5.1) (from Cryo I number 12, Emerald BioStructures).

All these crystals possessed a cryo-buffer in their reservoir solution and could be therefore directly frozen in a liquid nitrogen stream and subjected to data collection.

- The initial crystallization condition for the tmaL12 CTD:EF-Tu(Gd):GMPPNP putative crystals was Classics number 69 (Nextal Biotechnologies). Larger crystals were obtained with $0.05 \mathrm{M} \mathrm{KH}_{2} \mathrm{PO}_{4}, 25 \%$ PEG 8000. Further screening and testing of these crystals are ongoing.

\section{Data collection and processing}

- Initially, crystals of tmaL10:L12 were tested for their diffraction ability on the in-house source. Diffraction images were collected at $100 \mathrm{~K}$ on a Mar345 image plate (MarResearch, Eppendorf) equipped with a copper rotating anode generator (Nonius, Solingen). However, these crystals did not diffract X-Rays to high resolution (7-7.5 $)$. The diffraction pattern extended to $3.5 \AA$ for the crystals belonging to condition II and a complete data set was collected on a Mar225 CCD detector at Swiss Light Source synchrotron (Villigen, Switzerland). tmaL10:L12 crystals belonged to the R32 space group.

- Data sets for all three crystal forms of tmaL10:L12 NTD were collected at beamline BW6 (DESY, Hamburg) at 100K on a Mar-Research CCD detector. Anomalous data were recorded at four wavelengths around the selenium K-edge from a SeMetderivatized crystal of Form I. The crystal Form I diffracted to $2.3 \AA$ and belonged to the orthorhombic space group. The native tmaL10:(L12 NTD) 6 complexes yielded an orthorhombic and a monoclinic crystal forms that diffracted to $2.1 \AA$ and $1.9 \AA$ resolution, respectively. All complexes contained one L10 molecule and six L12 NTD molecules in the asymmetric unit. The X-Ray data were indexed and integrated with DENZO and scaled with SCALEPACK (Otwinowski and Minor 1996). 


\section{Phase generation, model building and refinement}

- Using peak-, inflection point-, and high energy remote data of the SeMet MAD experiment on a crystal Form I of tmaL10:L12 NTD, six selenium sites could be located and refined with Shelx D (Schneider and Sheldrick 2002). Initial phase calculations and solvent flattening to derive the hand of the heavy atom positions were carried out with Shelx E. The phases output from Shelx E were further refined with DM (Collaborative Computational Project, 1994), that generated a high quality electron density map. The chain autotracing was done with ARP/wARP (Morris et al. 2003). Model building was completed manually with MAIN (http://www-bmb.ijs.si/doc/index.html). The crystal Forms II and III were subsequently solved by molecular replacement (Collaborative Computational Project 1994) using the Form I structure coordinates. All three crystal forms were refined with CNS (Brunger et al. 1998) using simulated annealing, positional and temperature factor refinement protocols. The water molecules were placed automatically with CNS and checked manually in MAIN.

- tmaL10:L12 structure was solved by molecular replacement using the crystal structure of tmaL10:(L12 NTD) 6 as a search model. The L10 NTD and the L10 helix $\alpha 8$ $(\mathrm{L} 12 \mathrm{NTD})_{6}$ were used as separate parts. The structure did not reveal electron densities corresponding to the hinges and CTDs of L12.

\section{E. Structure analysis}

The molecular geometry of the tmaL10:L12 NTD structure was validated with PROCHECK (Laskowski et al. 1993). Figures were prepared with PyMol (http://pymol.sourceforge.net). The intermolecular contacts were analyzed using the Protein-Protein Interaction Server (http://www.biochem.ucl.ac.uk/bsm/PP/server/).

\section{Stoichiometry of L10:L12 complexes}

\section{A. Multiple sequence alignment}

The sequence alignment of bacterial L10 proteins and of archaeal L10E, yeast P0 and human P0 proteins was performed with Clustlal_X (Thompson et al. 1997), using default parameters, and displayed with ALSCRIPT (Barton 1993). 


\section{B. Multi-angle laser light scattering}

For molar mass measurements, purified tmaL10:L12, tmaL10:L12 NTD, tmaL10:L12 NTD/hinge, tmaL10:ecoL12, aaeL10:L12, ecoL10:L12 complexes (200 $\mu \mathrm{l}$ samples at $2 \mathrm{mg} / \mathrm{ml}$ in complex) were analyzed by asymmetric flow field-flow fractionation using an Eclipse F particle sizing system (Wyatt Technologies Corporation, USA), operated in phosphate buffered saline (PBS), $\mathrm{pH} 7.4$ (for a number of samples the measurement was repeated with a buffer containing $10 \mathrm{mM}$ Tris, $50 \mathrm{mM} \mathrm{NaCl} \mathrm{pH} \mathrm{8.0),} \mathrm{at}$ room temperature. Ultra pure BSA ( $2 \mathrm{mg} / \mathrm{ml})$ was used as a control. The L10:L12 complexes eluted as single peaks and were analyzed by multi-angle laser light scattering on a 18-angle DAWN EOS light scattering detector (Wyatt Technologies), equipped with a $30 \mathrm{~mW}$ Gallium-arsenide $690 \mathrm{~nm}$ laser light source and on an Optilab DSP differential interferometric refractometer (these results are summarized as an application note at http://www.wyatt.com/literature/ribozymesubunits.pdf). Data were analyzed using Astra software (Wyatt Technologies).

\section{CD spectroscopy studies of tmaL10:L12 complex}

Circular dichroism (CD) is commonly used in denaturation experiments in which the CD signal of a protein is monitored while the protein is perturbed in some fashion (e.g. increasing temperature, chemical denaturation). tmaL10:L12 $(0.1 \mathrm{mg} / \mathrm{ml})$ was measured in PBS, pH 7.4. The thermal melting profile (molar ellipticity values versus temperature) was monitored at $222 \mathrm{~nm}$ on a Jasco 720 spectropolarimeter (Gross-Umstadt) between $25^{\circ} \mathrm{C}$ and $85^{\circ} \mathrm{C}$. The heating rate was $60^{\circ} \mathrm{C} / \mathrm{h}$.

\section{Characterization of the interaction between tmaL12 CTD and elongation factors using Biacore}

Interaction studies between tmaL12 CTD and tmaEF-Tu(Gd) or tmaEF-G were performed on a Biacore X (Sweden). Biacore system exploits the surface plasmon resonance (SPR) as a detection principle to monitor the interaction between biomolecules in real time. The minute amounts in mass concentrations at the surface of the sensor chip as a consequence of the association and dissociation between the molecules is measured as an SPR response, 
and is displayed as a function of time on a graph known as sensorgram. The effects of EF$\mathrm{Tu}(\mathrm{Gd}), \mathrm{EF}-\mathrm{Tu}(\mathrm{Gd}): \mathrm{GTP}$ and EF-G on the binding to His 6 -tagged L12 CTD (that had the ability to covalently bind to a Ni-NTA sensor chip) were evaluated. The buffer of the system was $10 \mathrm{mM}$ HEPES, $150 \mathrm{mM} \mathrm{NaCl}, 0.005 \%$ Tween $20 \mathrm{pH} 7.4$ and was run at 10 $\mu \mathrm{l} / \mathrm{min}$. The EF-Tu(Gd):GTP complex was prepared in buffer A $(50 \mathrm{mM}$ Tris, $30 \mathrm{mM}$ $\mathrm{KCl}, 1 \mathrm{mM} \mathrm{MgCl}$, $\mathrm{pH}$ 7.6) as follows: $\mathrm{EF}-\mathrm{Tu}(84 \mathrm{nmol})$ was incubated with $1 \mathrm{mM} \mathrm{GTP,} 3$ $\mathrm{mM}$ phosphoenolpyruvate, $10 \mu \mathrm{g} / \mathrm{ml}$ pyruvate kinase for $15 \mathrm{~min}$ at $37^{\circ} \mathrm{C}$. Except for the His $_{6}$-L12 CTD:EF-Tu(Gd):GTP interaction (where approximately $200 \mathrm{nM}$ of both $\mathrm{His}_{6}$ L12 CTD and EF-Tu(Gd):GTP were used), all the experiments were performed with a concentration of $100 \mathrm{nM}$ for each protein.

\section{Calorimetric analysis of the tmaL11:L12 CTD complex}

The interaction between $t m a \mathrm{~L} 12 \mathrm{CTD}$ and $t m a \mathrm{~L} 11$ proteins was quantitated by isothermal titration calorimetry in a microcalorimeter (MicroCal Inc., UK). Prior measurements, both proteins were buffer exchanged with PD-10 columns in $20 \mathrm{mM} \mathrm{NaH} \mathrm{PO}_{4} / \mathrm{Na}_{2} \mathrm{HPO}_{4}, 150$ $\mathrm{mM} \mathrm{NaCl}, 1 \mathrm{mM}$ DTT, $\mathrm{pH} 7.0$ buffer and degassed. The solution of tmaL12 CTD (135 $\mu \mathrm{M}, 2.5 \mathrm{ml}$ ) was thermally equilibrated against the reference cell containing buffer at $20^{\circ} \mathrm{C}$. Next, 12 injections of a solution of tmaL11 (1 mM, $500 \mathrm{ml})$ were performed, and the energy required to reestablish thermal equilibrium between the two cells after each addition was measured and plotted in microcalories per second. Integration yielded the enthalpy of complex formation in kilocalories per mole. The stoichiometry of complex formation was calculated using the manufacturer's software.

\section{Preparation of Thermotoga maritima ribosomes}

Ribosomes from T. maritima MSB8 were prepared as described (Rodnina and Wintermeyer 1995), except for opening of the cells by a French press (T. maritima MSB8 cells were a kind gift from K.O. Stetter, Regensburg). 70S ribosomes were prepared as follows: frozen T. maritima MSB8 cells (50 g, wet weight) were opened by a French press (14000-16000 psi, the crude extract was passed twice onto the French press) in $100 \mathrm{ml}$ of buffer A $\left(20 \mathrm{mM}\right.$ Tris- $\mathrm{HCl}, \mathrm{pH}$ 7.6, $100 \mathrm{mM} \mathrm{NH}_{4} \mathrm{Cl}, 10 \mathrm{mM}$ magnesium acetate, $0.5 \mathrm{mM}$ EDTA, $3 \mathrm{mM} \beta$-mercaptoethanol containing DNase I (RNase-free; Boehringer) at 3 
$\mu \mathrm{g} / \mathrm{ml})$. The S-30 fraction was layered in portions of $16 \mathrm{ml}$ on $9 \mathrm{ml}$ of $1.1 \mathrm{M}$ sucrose in buffer B (20 mM Tris- $\mathrm{HCl}, \mathrm{pH} 7.6,0.5 \mathrm{M} \mathrm{NH}_{4} \mathrm{Cl}, 10 \mathrm{mM}$ magnesium acetate, $0.5 \mathrm{mM}$ EDTA, $3 \mathrm{mM} \beta$-mercaptoethanol). After centrifugation for $16 \mathrm{~h}$ at $33,000 \mathrm{rpm}$ in a Beckman Ti 50.2 rotor, ribosomes were washed by dissolving the pellets in $200 \mathrm{ml}$ of the same buffer, incubating for $2 \mathrm{~h}$, and sedimenting portions of $20 \mathrm{ml}$ through $1.5 \mathrm{ml}$ of $1.1 \mathrm{M}$ sucrose in the same buffer (Ti 50.2, $6 \mathrm{~h}, 45,000 \mathrm{rpm}$ ). The washing step was repeated twice, and the final centrifugation was made in a Beckman SW-28 rotor for $20 \mathrm{~h}$ at 28,000 rpm. Pellets were resuspended in buffer $\mathrm{C}\left(10 \mathrm{mM}\right.$ Tris- $\mathrm{HCl}, \mathrm{pH} 7.6,60 \mathrm{mM} \mathrm{NH}_{4} \mathrm{Cl}, 5$ $\mathrm{mM}$ magnesium acetate, $0.25 \mathrm{mM}$ EDTA, $3 \mathrm{mM} \beta$-mercaptoethanol) containing 5\% sucrose. The 70S tight couples were isolated by zonal centrifugation in a Beckman Ti15 rotor $(17 \mathrm{~h}, 28,000 \mathrm{rpm})$ on a convex exponential gradient from $10 \%$ to $37 \%$ sucrose $(1.4$ liters) in buffer C. The 70S peak was collected, and ribosomes were pelleted (Ti 50.2, $20 \mathrm{~h}$, 45,000 rpm), resuspended in buffer D (50 mM Tris- $\mathrm{HCl}, \mathrm{pH} 7.6,70 \mathrm{mM} \mathrm{NH}_{4} \mathrm{Cl}, 30 \mathrm{mM}$ $\mathrm{KCl}, 7 \mathrm{mM} \mathrm{MgCl}$, $1 \mathrm{mM} \mathrm{DTT}, 0.5 \mathrm{mM}$ EDTA), frozen in small portions in liquid nitrogen, and stored at $-80^{\circ} \mathrm{C}$. Ribosome concentrations were determined from absorption measurements on the basis of $23 \mathrm{pmol} / \mathrm{A}_{260}$ unit. Unless otherwise specified, all operations were performed at $4^{\circ} \mathrm{C}$.

\section{Measurement of the Thermotoga maritima ribosomal activity}

To prepare a 70S initiation complex, $0.4 \mu \mathrm{M}$ of $70 \mathrm{~S}$ ribosomes were incubated with a mix of $0.68 \mu \mathrm{M} E$. coli initiation factors (IF1, IF2, IF3), $0.8 \mu \mathrm{M} \mathrm{f}\left[\mathrm{H}^{3}\right] \mathrm{Met}^{-\mathrm{tRNA}}{ }^{\mathrm{fMet}}(3200$ dpm/pmol), $0.8 \mu \mathrm{M}$ of mRNA (Rodnina and Wintermeyer 1995), $1 \mathrm{mM} \mathrm{GTP}$, and up to $150 \mu \mathrm{l}$ buffer A (50 mM Tris, $70 \mathrm{mM} \mathrm{NH} 4 \mathrm{Cl}, 30 \mathrm{mM} \mathrm{KCl}_{2}, 7 \mathrm{mM} \mathrm{MgCl}_{2}, \mathrm{pH}$ 7.6). The reaction mixture was left at $37^{\circ} \mathrm{C}$ for 1 h. $25 \mu$ of this reaction corresponding to $10 \mathrm{pmol}$ of ribosomes were rapidly filtrated through a $0.45 \mu \mathrm{m}$ nitrocellulose filter (Sartorius, Göttingen), pre-equilibrated with buffer A. Next, the filter was washed extensively with buffer A and subsequently dissolved in a scintillation cocktail QS361 (Zinsser, Frankfurt). To measure the amount of $\mathrm{f}\left[\mathrm{H}^{3}\right]$ Met-tRNA bound to the ribosome, the ${ }^{3} \mathrm{H}$ radioactivity was monitored in a Packard 2500 scintillation counter. Activities of the ribosomes in partial reactions of initiation, as measured by nitrocellulose filtration, were $25.2 \%$ after $1 \mathrm{~h}$. 


\section{$\underline{\text { Results }}$}

\section{Expression screening of L10 and L12 proteins from different bacteria}

In order to produce a full-length L10:L12 complex, genes coding for the respective proteins were subcloned either individually in different expression vectors, or in the coexpression vector pETM-CoEx (EMBL, Heidelberg).

The rplJ and $r p l L$ genes coding for L10 and L12 proteins, respectively, from several thermophilic bacteria (Aquifex aeolicus, Thermus thermophilus and Thermotoga maritima) were selected for this study. Although most detailed functional studies of the protein synthesis mechanisms were carried out in E. coli, proteins from thermophilic organisms were chosen for their high thermostability and increased resistance to proteases. In addition, they appeared more suitable for crystallization and structural studies. Indeed, high resolution structures of the bacterial ribosome (Schluenzen et al. 2000; Wimberly et al. 2000; Yusupov et al. 2001), individual ribosomal proteins (Liljas and Garber 1995) and translation factors (Berchtold et al. 1993; Ævarsson et al. 1994) were derived from thermophilic organisms. Of particular importance for this study was the crystal structure of the isolated protein L12 from Thermotoga maritima, which yielded high quality crystals (Wahl et al. 2000a; Wahl et al. 2000b), in contrast to its E. coli ortholog, which disintegrated in time and provided high quality crystals only for the C-terminal part (Liljas et al. 1978).

Four E. coli cell strains were used as hosts for the expression of L10 and L12 proteins: BL21(DE3), BL21(DE3)pLysS, BL21(DE3)CodonPlus-RIL and Rosetta(DE3). An expression screening aiming to characterize the best vectors and cell strains for L10 and L12 protein production was performed. Its outcome is presented in Table 8. Based on this data, only few constructs leading to optimal results were selected for subsequent protein production and crystallization (described in detail in sections II and III). 


\begin{tabular}{|c|c|c|c|c|c|c|c|}
\hline \multirow[b]{2}{*}{ vectors } & \multicolumn{3}{|c|}{ aae } & \multicolumn{2}{|c|}{ tth } & \multicolumn{2}{|c|}{ tma } \\
\hline & L10 & L12 & L10:L12 & L10 & L12 & L10 & L12 \\
\hline pETM-CoEx & + & + & + & - & + & - & + \\
\hline pGEX6P1 & - & & & - & & - & \\
\hline pETM-10 & & & & + & & + & \\
\hline pETM-11 & & + & & + & & + & \\
\hline pETM-20 & & & & + & & + & \\
\hline pETM-30 & & & & + & & + & \\
\hline pETM-ZZ & & & & + & & + & \\
\hline
\end{tabular}

Table 8. Expression pattern of L10 and L12 proteins from several thermophilic organisms (aae: Aquifex aeolicus, tth: Thermus thermophilus, tma: Thermotoga maritima) in four different $E$. coli strains. The vectors used for gene expression are also indicated. (+) constructs leading to L10 and L12 expression in all four $E$. coli strains tested; (-) constructs that failed to yield any L10 or L12 activity in all four E. coli strains tested. In red are depicted the constructs which were further used for protein complex production and crystallization. Several clones of both tthL10 and tmaL10 in pETM-series vectors were produced together with U. Reidt, Cellular Biochemistry/X-Ray Crystallography Department, MPI for Biophysical Chemistry, Göttingen.

\section{L10:L12 complex from Aquifex aeolicus}

\section{A. Production of the protein complex}

As outlined in Table 8, an optimal expression of L10 (aaeL10) and L12 (aaeL12) proteins from Aquifex aeolicus was achieved using pETM-CoEx vector in Rosetta(DE3) cell strain.

Both aaeL10 and aae L12 proteins were independently produced without affinity tag and displayed high solubility and stability. To reconstitute the complex, cell lysates containing overexpressed aaeL10 and aaeL12 proteins were pooled and purified by heat treatment at $90^{\circ} \mathrm{C}$. This approach simplified subsequent chromatographic procedures, as most of $E$. coli proteins were denatured and precipitated. A similar purification procedure using heat treatment at $90^{\circ} \mathrm{C}$ was reported for EF-G from the same bacterium, without precipitation of the target protein (Martemyanov et al. 2000). Next, the protein complex was further purified via anion exchange chromatography, eluting at approximately 300 $\mathrm{mM} \mathrm{NaCl}$ from a DEAE column. The complex was subjected to size exclusion chromatography, concentrated, and used for crystallization trials. An overview of this purification procedure is presented in Figure 8. 

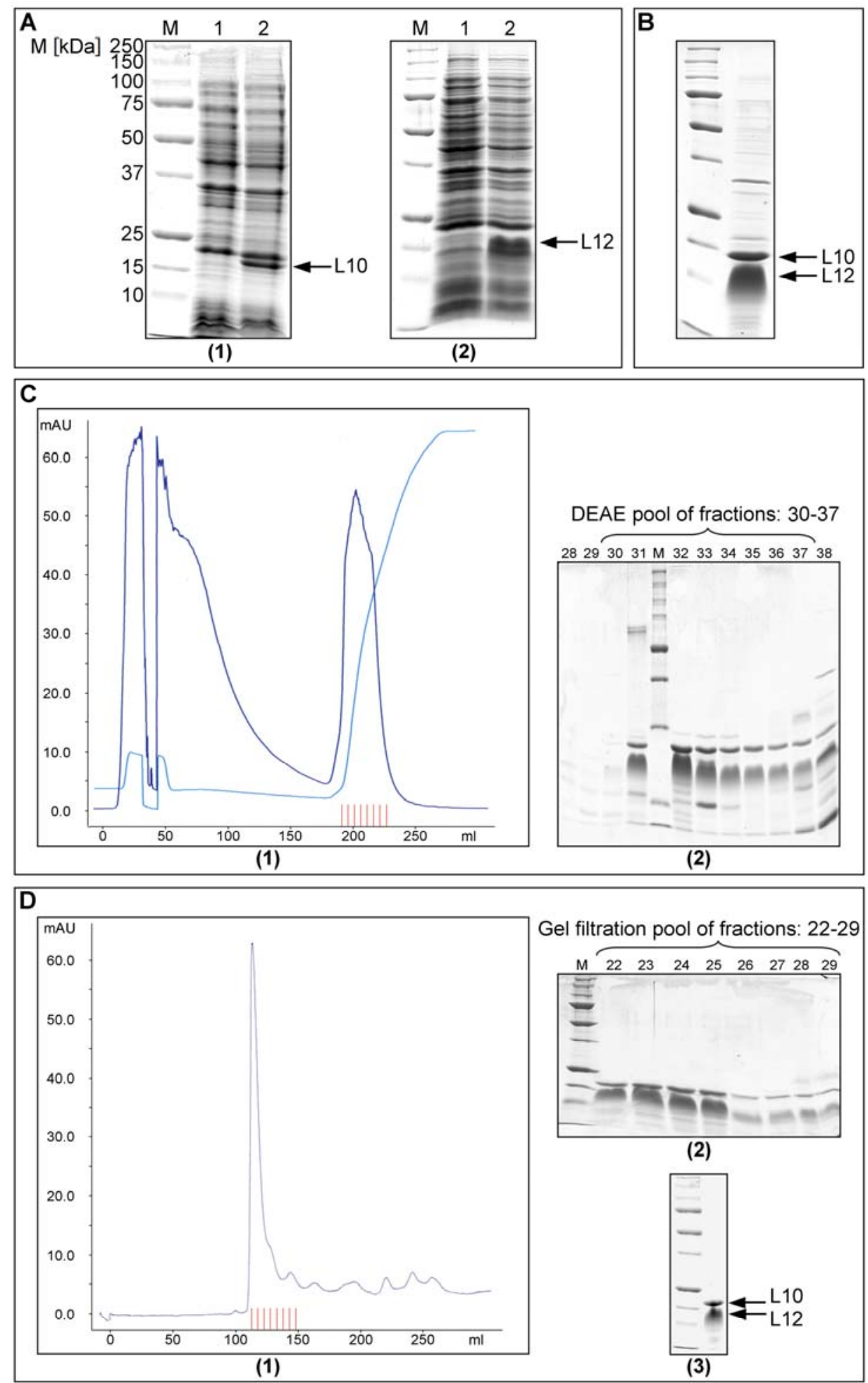

Figure 8. Expression and purification of L10:L12 protein complex from A. aeolicus. (A) (1) aaeL10 expression. Prior induction (lane 1) and post induction (lane 2) phases are indicated. (2) aaeL12 expression, labeling as for (1) (B) Reconstituted aaeL10:L12 complex after heating at $90^{\circ} \mathrm{C}$ step. (C) Anion exchange chromatography. (1) Chromatogram representing the elution profile of the aaeL10:L12 complex on a HiTrap DEAE column. On the x-coordinate is represented the volume [ml], whereas UV absorption at $280 \mathrm{~nm}$ $[\mathrm{mAU}]$ is provided on the ordinate. (2) Fractions containing purified aaeL10:L12 complex (30-37) were subjected to gel filtration. (D) Size exclusion chromatography. (1) Chromatogram representing the elution profile of the aaeL10:L12 complex on a HiLoad 26/60 Superdex 75 prep grade column. (2) Fractions containing the purified complex (22-29) were concentrated. (3) aaeL10:L12, final preparation control. 


\section{B. Validation of the complex formation}

In isolation, the thermophilic archaeon Sulfolobus solfataricus L10 and L12 proteins were found to form a very stable complex, that could not be disrupted even using high concentrations of denaturation agents such as $6 \mathrm{M}$ urea (Casiano et al. 1990). In the present work, the aaeL10:L12 complex formation was ascertained by (i) differences in heat stability of the L10:L12 complex, as compared to its constituent proteins, and (ii) gel filtration. By heating individual aae L10 and aaeL12 proteins to different temperatures ranging from 60 to $90^{\circ} \mathrm{C}$, different abilities to withstand high temperatures were evidenced. The isolated aae L10 protein was unstable, rapidly precipitating above $70^{\circ} \mathrm{C}$ (Figure 9A). Moreover, attempts to purify L10 were not successful due to its loss during purifications steps (data not shown). Conversely, aaeL12 protein was found very stable at all the tested temperatures (Figure 9B). This observation was in keeping with a previous experiment demonstrating the property of the sole L12 protein to retain some of its secondary structure elements even at extreme conditions of $\mathrm{pH}$ (its own $\mathrm{pI}$ is 4.9, suggesting a very acidic protein), temperature or $6 \mathrm{M}$ guanidinium hydrochloride (Luer and Wong 1980). Remarkably, the aaeL10:L12 complex was stable even at $90^{\circ} \mathrm{C}$, suggestive for its formation and stability (Figure 8B). The stabilization of the secondary and tertiary structures of L10, only when complexed to L12, was also observed using calorimetry and CD spectroscopy for the E. coli counterparts (Gudkov et al. 1978).

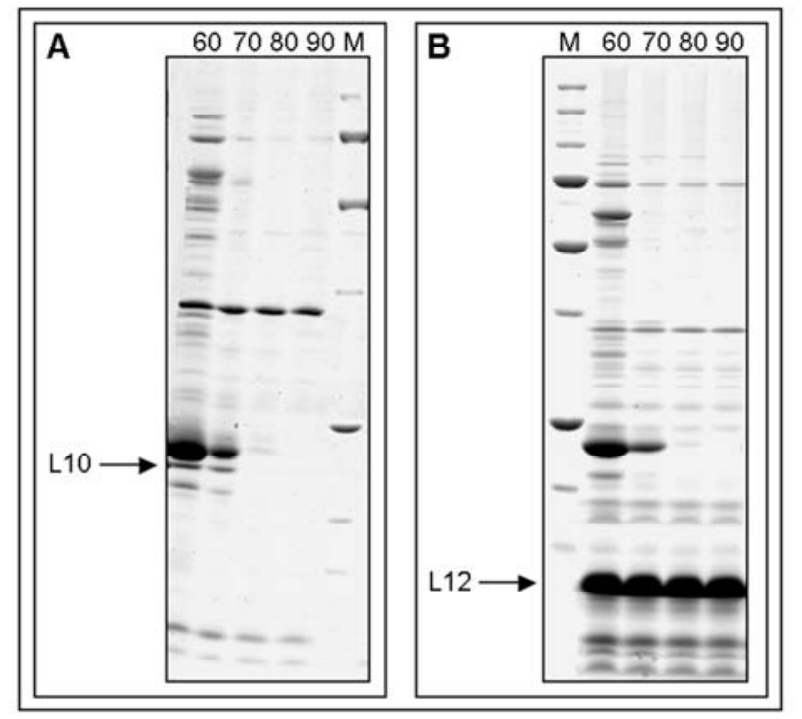

Figure 9. Heat treatment at $60,70,80,90^{\circ} \mathrm{C}$ of S-100 fractions containing the overexpressed proteins $\mathrm{L} 10$ or L12, respectively. (A) Heat treatment of protein L10 indicates a denaturation above $70^{\circ} \mathrm{C}$. (B) Conversely, L12 is stable at all the tested temperatures. 
In line with heat denaturation experiments, the gel filtration profile (Figure 8D-1) showed an early elution, indicative for the complex formation, rather than individual aae L10 and aaeL12, which are low-molecular weight r-proteins $(22.5$ and $13.5 \mathrm{kD}$, respectively), and therefore expected to exhibit a late elution.

\section{Crystallization trials}

The aaeL10:L12 protein complex $(20 \mathrm{mg} / \mathrm{ml})$ was subjected to crystallization by means of vapor diffusion. Mainly the sitting drop technique was employed. 288 conditions, covering a wide range of $\mathrm{pH}$, precipitants and additives, were screened (Table 7). The protein was found stable in numerous conditions employed for crystallization. Both 4 and $20^{\circ} \mathrm{C}$ temperatures were tested. Despite these attempts, as well as usage of several protein concentrations or different ratios of protein:reservoir in the drop, crystallization of the aaeL10:L12 complex proved to be unsuccessful.

\section{L10:L12 complex from Thermotoga maritima}

As the previous approach did not lead to the crystallization of the A. aeolicus L10:L12 complex, attempts to crystallize the same complex from T. maritima were made.

It is important to notice that crystallization of a bacterial L10:L12 full-length complex was reported more than two decades ago, however without resulting in a successfully determined structure (Liljas and Newcomer 1981). Additionally, in numerous crystal structures or cryo-EM maps, no electron density corresponding to the L10:L12 complex was found. The difficulty of disclosing the structure of this complex could derive from its inherent flexibility, especially at the level of the hinge region of L12, connecting the NTD with the CTD. Hence, in parallel with the crystallization of the full-length tmaL10:L12 complex, a strategy expected to limit the flexibility of tmaL10:L12 was designed. A complex encompassing the full-length L10 and only the N-terminal domains of L12 (L12 NTDs) was generated (for a schematic representation of both L10 and L12 proteins, see Figure 5). Protein L12 was trimmed after residue G30 in a small loop connecting the NTD and the hinge, thereby removing the flexible part of the complex, namely the hinges carrying the CTDs. 


\section{A. Production of protein complexes}

\section{Native tmaL10:L12 and tmaL10:L12 NTD complexes}

tmaL12 was obtained using a previously described clone (based on the pET22b(+) vector) (Wahl et al. 2000b), that allowed the protein production without affinity tag. To generate the tmaL12 NTD, a stop codon was introduced after codon 30 in the above mentioned plasmid (Wahl et al. 2000b) (Table 6). tmaL10 was cloned into the pETM-ZZ vector, which provides an $\mathrm{N}$-terminal $\mathrm{His}_{6} / \mathrm{ZZ}$ double tag (Table 8).

tmaL10 and either full-length tmaL12 or tmaL12 NTD were co-expressed after cotransformation into E. coli Rosetta(DE3) cell strain. Both co-expressed tmaL10:L12 and tmaL10:L12 NTD complexes were purified via affinity chromatography on NickelNitrilotriacetate (Ni-NTA) beads, through the His 6 -tag attached to the tmaL10 N-terminus. The protein complexes were eluted from the Ni-NTA resin in $300 \mathrm{mM}$ imidazole. Following a buffer exchange step and the removal of the tag with tobacco etch virus (TEV) protease, the complexes were re-purified on Ni-NTA beads. Further heat treatment at $80^{\circ} \mathrm{C}$ and size exclusion chromatography yielded highly pure proteins. An overview of the tmaL10:L12 and tmaL10:L12 NTD expression and purification procedures is provided in Figure 10.

Due to its predominantly acidic amino acids composition (the calculated pI of tmaL12 is 4.8 ), the $13.5 \mathrm{kD}$ tmaL12 protein migrated slower than expected on SDS-PAGE gels. The migration appeared to be concentration dependent: the more concentrated the protein, the slower the migration. In addition, tmaL12 also lacks aromatic residues, in particular Tryptophan, resulting in a decreased absorbance at $280 \mathrm{~nm}$. Similarly, protein concentration was found considerably underestimated by other conventional methods, e.g. Bradford assay. Indeed, previous quantitative amino acid analysis of this protein indicated a 6-7 fold increase in concentration as compared to a Bradford concentration determination (Wahl et al. 2000b). This information, as well as in-gel comparisons with proteins of known concentrations, allowed the concentration estimation of the L10:L12 complex. 
$\underline{\text { Results }}$
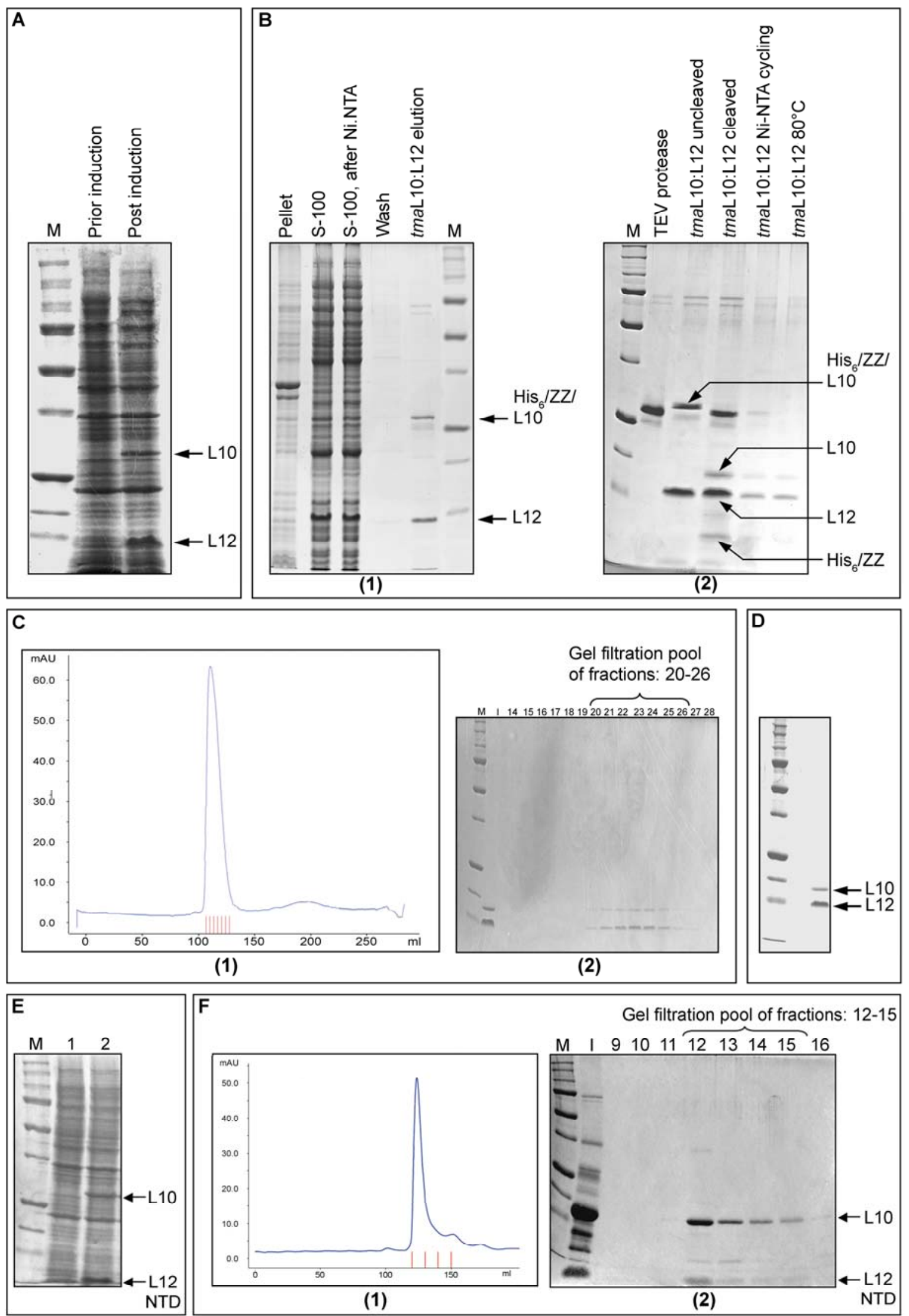
Figure 10. L10:L12 protein complex from T. maritima: expression and purification procedures. (A) tmaL10:L12 complex co-expression. (B) (1) Purification via affinity chromatography on Ni-NTA beads: the pellet of the cell lysate exhibited minute amounts of the protein complex, indicative of its solubility; S-100 fraction representing the supernatant of the cell lysate and demonstrating the presence of the soluble protein complex; S-100 after Ni-NTA corresponds to the flow-through of the cell lysate passed onto Ni-NTA beads; wash represents the flow-through of the washing of Ni-NTA beads; tmaL10:L12 elution corresponds to the elution of the protein complex from the Ni-NTA beads, demonstrating the presence of both $\mathrm{His}_{6} / \mathrm{ZZ/L10}$ and L12. (2) TEV protease control sample; TEV protease cleavage of the His 6 /ZZ tags; tmaL10:L12 Ni-NTA cycling: re-purification on Ni-NTA beads to remove $\mathrm{His}_{6} / \mathrm{ZZ}$ tag and TEV protease; heating at $80^{\circ} \mathrm{C}$ of the protein complex (C) Size exclusion chromatography. (1) Chromatogram representing the elution profile of the tmaL10:L12 complex on the Superdex 75 gel filtration column. (2) Fractions containing the purified complex (20-26) were further concentrated; I, input protein representing the complex before gel filtration. (D) $t m a$ L10:L12, final preparation. (E) tmaL10:L12 NTD complex co-expression. Prior induction (lane 1) and post induction (lane 2) phases are indicated. (F) (1) Chromatogram representing the elution profile of the tmaL10:L12 NTD complex on the Superdex 75 gel filtration column, as the last purification step. (2) Fractions containing the purified complex (12-15) were further concentrated and subjected to crystallization trials.

\section{Selenomethionine-derivatized tmaL10:L12 NTD complex}

To allow structure solution by multiwavelength anomalous dispersion (MAD), a selenomethionine (SeMet) substituted complex was also produced. The tmaL10 protein contains three methionines (N-terminal residue included) and tmaL12 NTD possesses one N-terminal methionine. The selenomethionine-containing protein complex was expressed in the E. coli B834(DE3)pLysS strain. These cells, being methionine-auxotroph, can only incorporate the selenomethionine subsequently supplemented in the medium. The yield of expression was slightly lower as compared to the wild-type, which is often seen in SeMetderivatized protein production. Subsequently, the complex was purified as described for the tmaL10:L12 NTD native complex (see previous chapter), concentrated to approximately $8 \mathrm{mg} / \mathrm{ml}$ and subjected to crystallization.

\section{B. Thermostability of the tmaL10:L12 complex}

One of the purification steps of the tmaL10:L12 complex consisted in heating at $80^{\circ} \mathrm{C}$. In order to test the stability of the purified complex at this temperature, the potential modification in protein folding induced by temperature was monitored at $222 \mathrm{~nm}$ on a Jasco 720 spectropolarimeter. The protein complex was in a physiological buffer (PBS, pH 7.4). Previously, it was shown that the tmaL12 protein exhibits a melting temperature of approximately $110^{\circ} \mathrm{C}$ at $\mathrm{pH} 7.5$, compared to $\sim 60^{\circ} \mathrm{C}$ for the highly homologous $E$. coli protein (Wahl et al. 2000b). Herein, due to instrument limitations, the data acquisition could not be performed above $85^{\circ} \mathrm{C}$. However, the negative molar ellipticity recorded until 
this temperature exhibited constant values, indicating no changes (e.g. denaturation) in the protein structure (Figure 11).

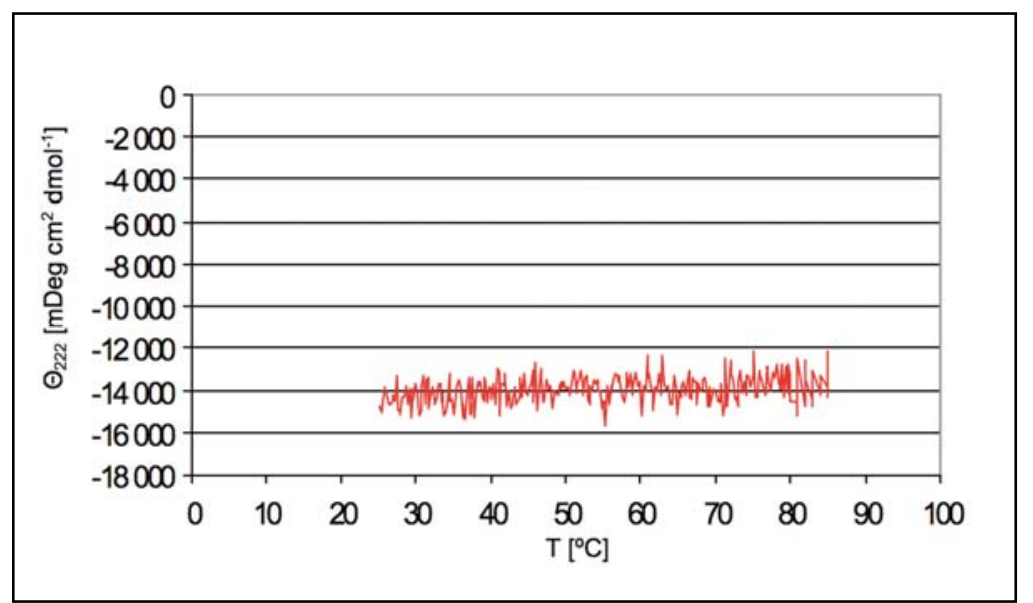

Figure 11. Thermal melting profile of the tmaL10:L12 complex between 25 and $85^{\circ} \mathrm{C}$. The molar ellipticity value was found constant with the temperature, consistent with an absence of modifications in the tmaL10:L12 structure.

\section{Crystallization}

\section{1. tmaL10:L12 crystals}

In order to crystallize the tmaL10:L12 complex, high-throughput crystallization was performed on the in-house vapor diffusion sitting drop dispensing apparatus. 1344 different conditions were screened using both sparse matrices and screens of ammonium sulfate and sodium malonate versus $\mathrm{pH}$. Three-dimensional crystals appeared after 7 days in several conditions which contained PEG 3350 as precipitant. The screening was performed at both 4 and $20^{\circ} \mathrm{C}$, but crystals grew better at $20^{\circ} \mathrm{C}$. In all the cases, single or aggregated needle-shaped crystals appeared. After optimization of $\mathrm{pH}$ and buffers, two conditions gave rise to single crystals. Condition I contained $0.2 \mathrm{M} \mathrm{NaCl}, 0.1 \mathrm{M}$ Tris $\mathrm{pH}$ 8.0, 20\% PEG 3350 and condition II (Figure 12A) $0.4 \mathrm{M}$ ammonium acetate, $\mathrm{pH} 7.2,25 \%$ PEG 3350, respectively.

However, attempts to reproduce these crystals from complexes prepared with a higher concentration of protease inhibitors were not successful. This finding points to the notion that the initially obtained crystals did not encompass the full-length tmaL10:L12 complex, but rather a trimmed variant, resulting from in situ proteolysis. Spontaneous cleavage was previously observed in the crystal structure of T. maritima L12 (Wahl et al. 2000a) and in crystallization trials of E. coli L12 (Liljas et al. 1978). Indeed, the full- 
length L12 seemed to be quantitatively reduced as estimated by the SDS-PAGE gel examination of washed crystals dissolved in loading buffer. This result suggested that a part of the protein was degraded (Figure 12B).

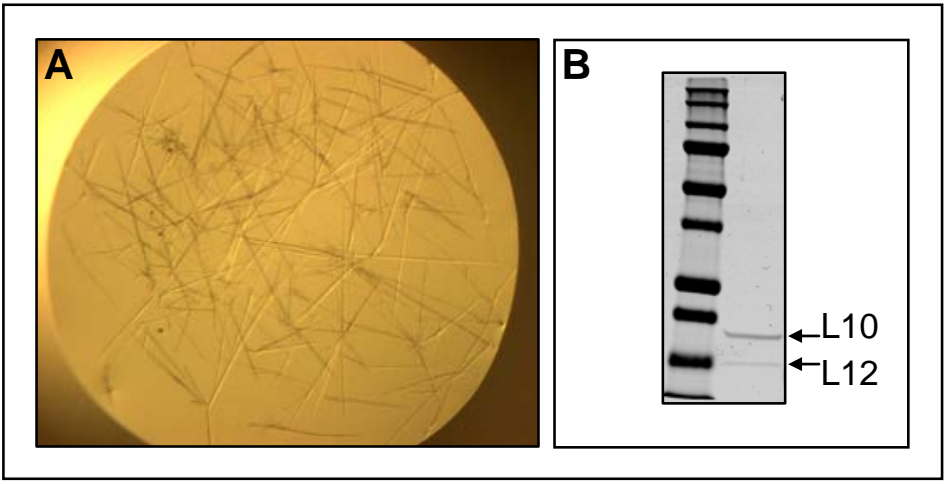

Figure 12. (A) The morphology of tmaL10:L12 crystals. (B) tmaL10:L12 crystals were repeatedly washed with reservoir solution in order to remove the non-crystallized protein from the drop, dissolved in loading buffer and subjected to SDS-PAGE. Note the weak amount of L12, suggesting a potential degradation due to proteolysis.

\section{Three crystal forms of the tmaL10:L12 NTD complex}

The tmaL10:L12 NTD complex, concentrated in a low salt buffer at $10 \mathrm{mg} / \mathrm{ml}$, was subjected to crystallization by means of vapor diffusion. 384 different conditions were screened, using sparse matrices from Nextal Biotechnologies and Hampton Research. Several crystallization conditions with MPD and ethylene glycol as precipitants yielded single, small, needle-shaped crystals. They grew within a day at $20^{\circ} \mathrm{C}$. One of these conditions, i.e. Cryo number 34 (Emerald BioStructures), gave three-dimensional crystals of excellent quality, which could be directly used for data collection. Subsequent buffer and $\mathrm{pH}$ optimization were required for some of the formulations, in order to produce single, large crystals. Next, the best conditions were probed for the selenium derivatized protein. After improvement steps, three crystals forms were used for data collection: one SeMet (Form I) (Figure 13A) and two native (Forms II and III) (Figure 13B, C). All the crystals, already cryo-protected from the reservoir solution, could be directly frozen in a liquid nitrogen stream. 

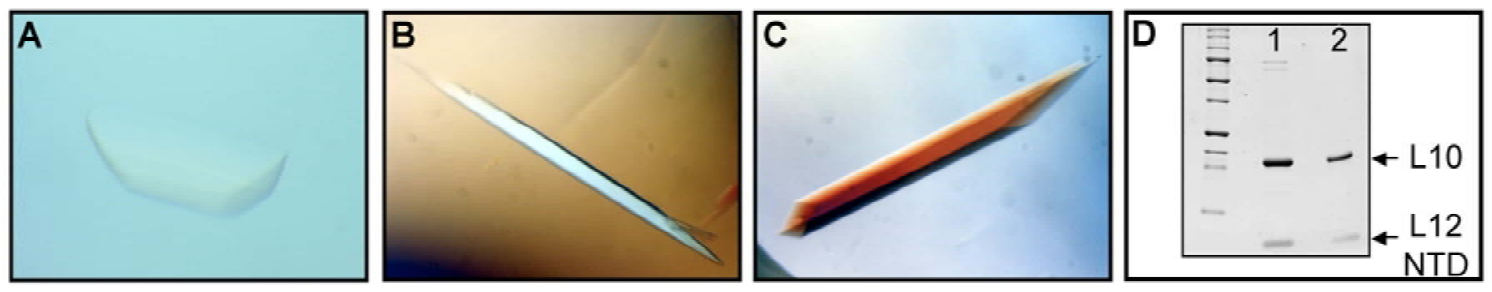

Figure 13. Crystal forms of tmaL10:L12 NTD complex. (A) Crystal Form I (SeMet-derivative). (B) A needle-shaped crystal Form II (native), visualized in polarized light. (C) A needle-shaped crystal Form III (native), visualized in polarized light. (D) Protein (lane 1) and crystals of tmaL10:L12 NTD dissolved in loading buffer (lane 2), subjected to SDS-PAGE and subsequently to mass spectrometry, for authentication.

\section{Data collection and processing}

\section{1. tmaL10:L12 complex}

Prior to the measurement of diffraction data, suitable crystals from conditions I and II were mounted with an adequately sized loop and directly frozen in a cold nitrogen stream. A complete data set of tmaL10:L12 crystals was collected on a synchrotron (SLS, Villigen, Switzerland). These crystals diffracted to $3.5 \AA$ and belonged to the primitive rhombohedral (R32) space group. The data collection statistics are summarized in Table 9.

\begin{tabular}{cc}
\hline \hline Data collection & \\
\hline \hline Space group & $\mathrm{R} 32$ \\
Unit cell $\left(\AA,{ }^{\circ}\right)$ & \\
$\mathrm{a}$ & 130.8 \\
$\mathrm{~b}$ & 130.8 \\
$\mathrm{c}$ & 64 \\
$\alpha$ & 90 \\
$\beta$ & 90 \\
$\gamma$ & 120 \\
Wavelength $\left.(\AA)^{(}\right)$ & 1.05 \\
Resolution $(\AA)$ & $30.0-3.5(3.6-3.5)$ \\
Unique reflections $^{(2728(226)}$ \\
Redundancy $_{\text {Completeness }(\%)}$ & 2.5 \\
$\mathbf{I} / \boldsymbol{\sigma}(\mathbf{I})$ & $12.8(1.5)$ \\
$\mathbf{R}_{\text {sym }}{ }^{\mathrm{a}}(\%)$ & $10.2(83.4)$ \\
\hline \hline
\end{tabular}

Table 9. Data collection statistics for the tmaL10:L12 crystal. Values for the last $0.1 \AA$ between brackets. ${ }^{\mathrm{a}} \mathrm{R}_{\mathrm{sym}}(\mathrm{I})=\left(\Sigma_{\mathrm{hkl}} \Sigma_{\mathrm{i}}\left[\left|I_{\mathrm{i}}(\mathrm{hkl})-<I(\mathrm{hkl})>\right|\right] / \Sigma_{\mathrm{hkl}} \Sigma_{\mathrm{i}}\left[I_{\mathrm{i}}(\mathrm{hkl})\right] ; I_{\mathrm{i}}(\mathrm{hkl})-\right.$ intensity of the $\mathrm{i}^{\text {th }}$ measurement of hkl; $<I(\mathrm{hkl})>-$ average value of hkl for all i measurements. 


\section{2. tmaL10:L12 NTD complexes}

Data sets for all three crystal forms were collected on a synchrotron (DESY, Hamburg, Germany). Crystals of Form I, II and III diffracted to 2.3, 2.1 and $1.9 \AA$ resolution, respectively. Anomalous data were recorded at four wavelengths (0.9793, $0.9795,0.95,1.05)$ around the selenium absorption-edge from a SeMet-derivatized crystal of Form I. The latter crystals belonged to an orthorhombic space group (P2 $\left.{ }_{1} 2{ }_{1} 2_{1}\right)$ with unit cell dimensions of $a=84.9 \AA, b=84.9 \AA, c=63.9 \AA, \alpha=\beta=\gamma=90^{\circ}$. The native Form II and III crystals belonged to the orthorhombic $\left(\mathrm{P} 2{ }_{1} 2_{1} 2_{1}\right)$ and monoclinic $\left(\mathrm{P} 2_{1}\right)$ space groups, respectively. Data statistics are summarized in Table 10.

\begin{tabular}{|c|c|c|c|c|c|c|}
\hline Crystal Form & $\mathbf{I}$ & & & & II & III \\
\hline Data collection & Peak & Infl. Point & $\begin{array}{c}\text { HE } \\
\text { Remote }^{\mathrm{a}} \\
\end{array}$ & $\begin{array}{c}\text { LE } \\
\text { Remote }^{a} \\
\end{array}$ & & \\
\hline Space group & $\mathrm{P} 2{ }_{1} 2_{1} 2_{1}$ & & & & $\mathrm{P} 2{ }_{1} 2_{1} 2_{1}$ & $\mathrm{P} 2_{1}$ \\
\hline \multicolumn{7}{|l|}{ Unit cell $\left(\AA,{ }^{\circ}\right)$} \\
\hline a & 84.9 & & & & 45.0 & 43.4 \\
\hline $\mathrm{b}$ & 84.9 & & & & 50.5 & 60.4 \\
\hline $\mathrm{c}$ & 63.9 & & & & 179.1 & 83.4 \\
\hline$\beta$ & & & & & & 91.9 \\
\hline Wavelength $(\AA)$ & 0.9793 & 0.9795 & 0.95 & 1.05 & 1.05 & 1.05 \\
\hline Resolution $(\AA)$ & $99.0-2.62$ & $99.0-2.62$ & $99.0-2.55$ & $30.0-2.2$ & $30.0-2.1$ & $30.0-1.9$ \\
\hline Processing & Anomalous & Anomalous & Anomalous & Normal & Normal & Normal \\
\hline Unique reflections & 26365 & 26407 & 29005 & 24019 & 24199 & 34091 \\
\hline Redundancy & 7.6 & 4.2 & 4.1 & 6.1 & 4.6 & 4.3 \\
\hline Completeness (\%) & $99.3(97.5)$ & $99.3(96.3)$ & $99.5(98.6)$ & $99.4(98.8)$ & $97.8(96.7)$ & $99.8(99.9)$ \\
\hline $\mathbf{I} / \sigma(\mathbf{I})$ & $34.8(5.3)$ & $24.6(3.4)$ & $23.8(8.4)$ & $27.6(1.8)$ & $21.6(2.6)$ & $35.4(3.6)$ \\
\hline $\mathbf{R}_{\text {sym }}^{\mathrm{b}}(\%)$ & $6.2(33.9)$ & $6.6(36.0)$ & $6.9(47.4)$ & $7.9(56.1)$ & $9.6(32.7)$ & $5.1(21.7)$ \\
\hline
\end{tabular}

Table 10. Data collection statistics for tmaL10:(L12 NTD $)_{6}$ crystals. Values for the last $0.1 \AA$ between brackets. ${ }^{\mathrm{a}} \mathrm{HE}-$ high energy; LE - low energy. ${ }^{\mathrm{b}} \mathrm{R}_{\text {sym }}(\mathrm{I})=\left(\Sigma_{\mathrm{hk}} \Sigma_{\mathrm{i}}\left[\left|I_{\mathrm{i}}(\mathrm{hkl})-<I(\mathrm{hkl})>\right|\right] / \Sigma_{\mathrm{hkl}} \Sigma_{\mathrm{i}}\left[I_{\mathrm{i}}(\mathrm{hkl})\right] ; I_{\mathrm{i}}(\mathrm{hkl})\right.$ - intensity of the $\mathrm{i}^{\text {th }}$ measurement of $\mathrm{hkl} ;<I(\mathrm{hkl})>-$ average value of hkl for all $\mathrm{i}$ measurements 


\section{E. Structure determination}

\section{1. tmaL10:(L12 NTD) 6 complexes}

The structure of Form I crystal could be solved by a four-wavelength Multiple Anomalous Diffraction (MAD) strategy (Table 11). Six selenium sites could be identified in the anomalous difference Fourier maps.

\begin{tabular}{lccc}
\hline \hline Phasing & \multicolumn{3}{c}{ Crystal Form I } \\
\hline \multicolumn{1}{l}{ Resolution $(\AA)$} & $20.0-2.62$ & $20.0-2.62$ & $20.0-2.55$ \\
Heavy atom sites & 6 & 6 & 6 \\
$\begin{array}{l}\text { Correlation } \\
\text { coefficients }(C C)\end{array}$ & & & \\
Overall & & & \\
Map & & 0.39 \\
Free & & 0.80 \\
FOM $^{\mathrm{c}}$ & & & 0.62 \\
\hline \hline
\end{tabular}

Table 11. Phasing statistics for the tmaL10:(L12 NTD $)_{6}$ crystal Form I. ${ }^{\mathrm{a}} \mathrm{HE}-$ high energy. ${ }^{\mathrm{b}} \mathrm{CC}=$ $\left[\Sigma w E_{0} E_{\mathrm{c}} \Sigma w-\Sigma w E_{\mathrm{o}} \Sigma w E_{\mathrm{c}}\right] /\left\{\left[\Sigma w E_{\mathrm{o}}{ }^{2} \Sigma_{w^{-}}-\left(\Sigma w E_{\mathrm{o}}\right)^{2}\right]\left[\Sigma w E_{\mathrm{c}}{ }^{2} \Sigma w-\left(\Sigma w E_{\mathrm{c}}\right)^{2}\right]\right\}^{1 / 2} ; w-$ weight (see http://shelx.uniac.gwdg.de/SHELX/shelx_de.pdf for full definitions). ${ }^{c} \mathrm{FOM}=$ Figure of merit $=\left[\left|F(\mathrm{hkl})_{\text {best }}\right|\right] /|F(\mathrm{hkl})|$; $\mathbf{F}(\mathrm{hkl})_{\text {best }}=\Sigma_{\alpha}\left[P(\alpha) \mathbf{F}_{\text {hkl }}(\alpha)\right] / \Sigma_{\alpha}[P(\alpha)]$

Further density modification with DM (Collaborative Computational Project, 1994) generated a high quality electron density map (Figure 14), which could be partly interpreted by automated model building with ARP/wARP (Morris et al. 2003), and subsequently completed manually using MAIN (http://www-bmb.ijs.si/doc/index.html). Next, structures of the other two crystal forms were determined by molecular replacement using as a search model the structure derived from crystal Form I. In all three structures, the asymmetric unit contained one molecule of full length L10 complexed with three dimers of L12 NTD. 


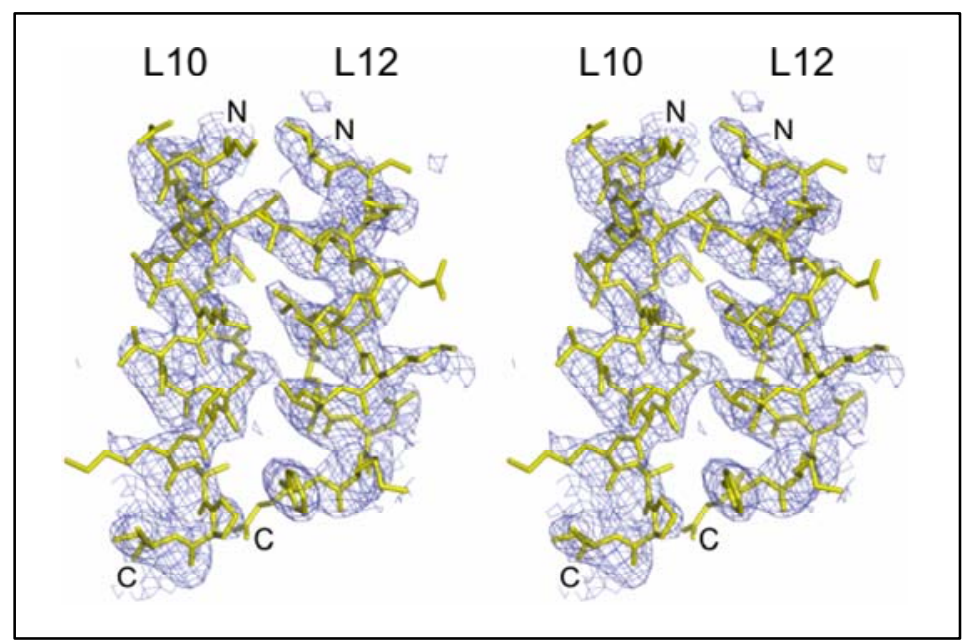

Figure 14. Stereo view of the experimental SeMet-MAD electron density map of the tmaL10:(L12NTD)6 after solvent flattening contoured at the $1 \sigma$ level. The density covers part of L10 helix $\alpha 8$ in contact with an L12 NTD helix, which was chosen as a representative portion of the complex (data phased up to $2.5 \AA$ resolution). The final atomic model (of crystal Form I) is superimposed for comparison (yellow sticks).

\section{2. tmaL10:L12 complex}

The structure of the tmaL10:L12 complex was solved by molecular replacement, in which the L10 NTD and the L10 helix $\alpha 8$-(L12 NTD)6 were used as separate parts (see structure description of the tmaL10:(L12 NTD) 6 complex, section III.G.1.)

The resulting electron density did not reveal features beyond the Glycine 30 of L12. Electron density for the L12 hinge region and the CTD was missing. Therefore, this structure can be regarded as another tmaL10:(L12 NTD) 6 truncated complex, in a different crystal form, which was generated by the spontaneous proteolysis of the full-length tmaL10:L12 complex.

\section{F. Refinement and quality of the model of the tmaL10:(L12 NTD) 6 crystal structures}

Models were refined to comparable working and free R-factors. The refinement parameters are presented in Table 12. The crystal Form I was refined to $2.3 \AA$ resolution, with an R-free value of 0.272. Similarly, crystals Forms II and III were refined with an Rfree value of 0.286 and 0.274 , respectively. 


\begin{tabular}{|c|c|c|c|}
\hline \multicolumn{4}{|l|}{ Refinement } \\
\hline Crystal Form & $\mathbf{I}$ & II & III \\
\hline Resolution $(\AA)$ & $20.0-2.3$ & $20.0-2.1$ & $20.0-1.9$ \\
\hline \multicolumn{4}{|l|}{ Model atoms } \\
\hline Protein & 2822 & 2807 & 2822 \\
\hline Water oxygens & 223 & 322 & 413 \\
\hline $\mathbf{R}_{\text {work }}{ }^{\mathrm{a}}(\%)$ & 22.2 & 21.2 & 22.6 \\
\hline $\mathbf{R}_{\text {free }}{ }^{\mathrm{b}}(\%)$ & 27.2 & 28.6 & 27.4 \\
\hline \multicolumn{4}{|l|}{ RMSD $^{\mathrm{e}}$ from ideality } \\
\hline Bond lengths $(\AA)$ & 0.006 & 0.006 & 0.007 \\
\hline Bond angles $\left({ }^{\circ}\right)$ & 1.13 & 1.10 & 1.17 \\
\hline \multicolumn{4}{|l|}{ Bonded B-factors $\left(\AA^{2}\right)$} \\
\hline Main chain & 1.6 & 3.0 & 1.5 \\
\hline Side chain & 2.7 & 4.8 & 2.3 \\
\hline Wilson B-factor $\left(\AA^{2}\right)$ & 47.4 & 36.7 & 39.8 \\
\hline \multicolumn{4}{|l|}{ Model B-factors $\left(\AA^{2}\right)$} \\
\hline Protein & 63.7 & 50.1 & 52.2 \\
\hline Water & 66.2 & 59.8 & 67.3 \\
\hline \multicolumn{4}{|l|}{$\phi / \psi(\%)$} \\
\hline Core & 95.4 & 96.6 & 98.1 \\
\hline Additionally allowed & 4.3 & 3.4 & 1.5 \\
\hline Generally allowed & 0.3 & 0 & 0 \\
\hline Disallowed & 0 & 0 & 0.3 \\
\hline
\end{tabular}

Table 12. Refinement statistics for tmaL10:(L12 NTD) 6 crystals. $\left.{ }^{a} R_{\text {work }}=\Sigma_{\text {hkl }}||\left|F_{\text {obs }}\right|-k\left|F_{\text {calc }}\right| \mid\right] /$ $\Sigma_{\mathrm{hkl}}\left[\left|\mathrm{F}_{\mathrm{obs}}\right|\right] \cdot{ }^{\mathrm{b}} \mathrm{R}_{\text {free }}=\Sigma_{\mathrm{hkl} \subset \mathrm{T}}\left[|| \mathrm{F}_{\mathrm{obs}}|-k| \mathrm{F}_{\text {calc }}||\right] / \Sigma_{\mathrm{hkl} \subset \mathrm{T}}\left[\left|\mathrm{F}_{\text {obs }}\right|\right] ;$ hkl $\subset \mathrm{T}-$ test set.

The Ramachandran plot of the crystal Form I is shown in Figure 15.

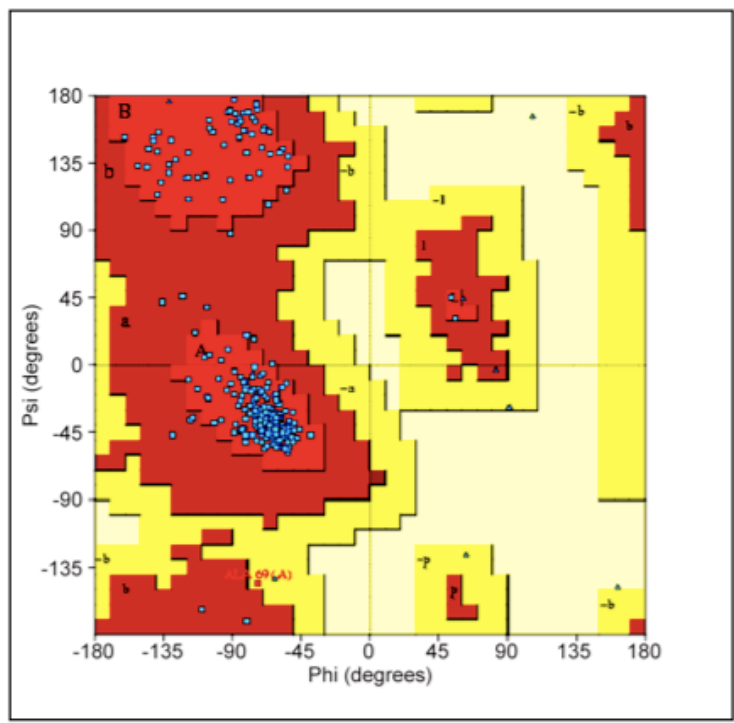

Figure 15. Ramachandran plot corresponding to the crystal Form I of tmaL10:(L12 NTD) 6 structure, calculated with PROCHECK (Laskowski et al. 1993). The red area depicts the most favorable region, the dark red area is the additionally allowed region and the dark yellow area is the generously allowed region. The triangles symbolize glycines or prolines. More than $90 \%$ of the residues are in the most favorable region, indicating a satisfactory geometry of the model. 
The model exhibits a good overall stereochemistry, with $95.4 \%$ of the residues in the most favored region, $4.3 \%$ in the additionally allowed region and one residue in the generously allowed region.

The structures were deposited in the Protein Data Bank (http://www.rscb.org/pdb), accession codes: 1ZAW (Crystal Form I), 1ZAX (Crystal Form II), 1ZAV (Crystal Form III).

\section{G. Crystal structure of the tmaL10:(L12 NTD) ${ }_{6}$ complex}

\section{Overall structure}

In all three crystal structures of the tmaL10:L12 $\mathrm{NTD}_{6}$ complex, one molecule of full length L10 was found in complex with six copies of L12 NTD, the latter forming three dimers (Figure 16). Protein L10 displays a globular N-terminal domain, followed by a long C-terminal $\alpha$-helix. A flexible loop connects these domains. The L10 NTD exhibits an $\alpha / \beta$ fold by which this protein is anchored to the 23S rRNA. The C-terminal helix ( $\alpha 8, \mathrm{~K} 137-$ K174) of L10 is kinked twice, at residues P151 and G161, resulting in three ten-residue segments. Each segment associates with one L12 NTD dimer through a five-helix bundle. Thus, the L10-L12 interaction region is characterized by a repetition of three almost identical helix $\alpha 8$-L12 NTD dimer elements. The binding of L12 to the C-terminal portion of L10 is in agreement with early observations that L10 exhibits two functionally different regions: one for binding the 23S rRNA and one for binding L12 (Pettersson 1979).

Each L12 NTD molecule contains two $\alpha$-helices connected by a short loop. Within each dimer, two L12 NTD molecules are entangled in an antiparallel fashion by extensive hydrophobic contacts, consistent with the previous observation that the N-terminal part of L12 is responsible for dimerization (Gudkov and Behlke 1978). 


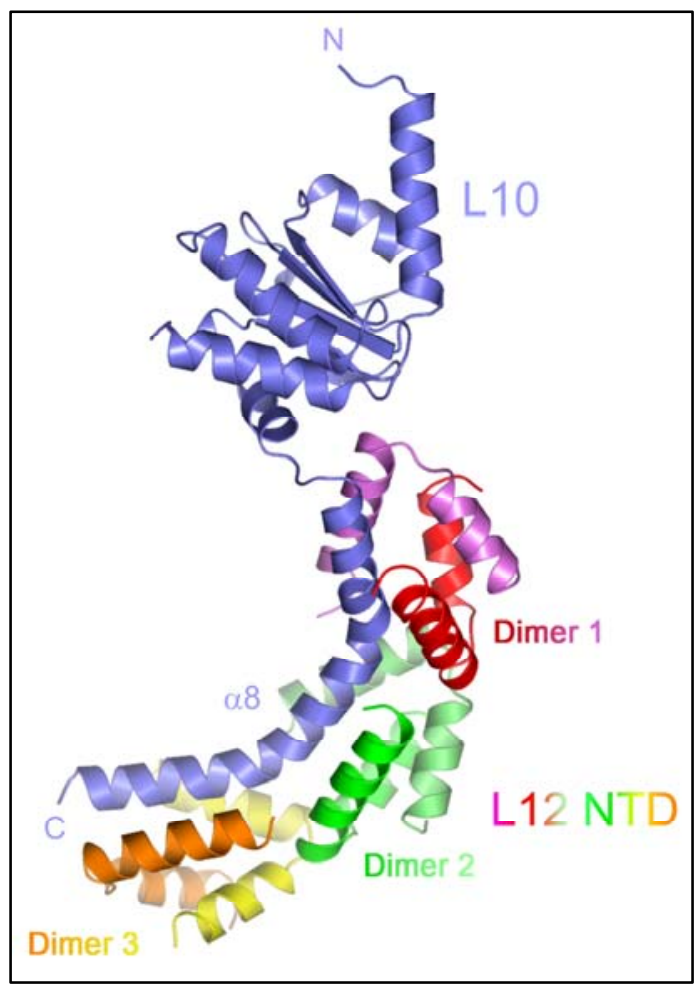

Figure 16. Overall structure of the tmaL10:(L12 NTD) ${ }_{6}$ complex showing the L10 NTD at the top and three L12 NTD dimers (protomers colored pink/red, light green/dark green, or yellow/orange, respectively) bound to the C-terminal helix $\alpha 8$ of L10 (blue) at the bottom.

\section{Detailed insights into the L10-L12 interaction}

Interfaces of the L12 NTD dimers with L10 bury about $1500 \AA^{2}$ of combined surface area each. The mode of interaction of the L12 NTD dimers with L10 is similar in each case and is dominated by hydrophobic contacts (Figure 17A). Precisely, 80\% of the interface residues are hydrophobic. Shape complementarity and electrostatic interactions at the periphery, such as salt bridges, hydrogen bonds and bridging water molecules, register the L12 NTD dimers on L10 helix $\alpha 8$ (Figure 17B). Helix $\alpha 8$ of L10 presumably adopts a regular structure only upon interaction with L12, consistent with the observation that L10 in isolation exhibits a decreased stability (herein and (Gudkov et al. 1978)) Thus, resembling the architecture of other ribosomal proteins, which use long extensions for intimate interactions with rRNA (Ban et al. 2000), L10 employs a C-terminal extension to interact with another protein. 


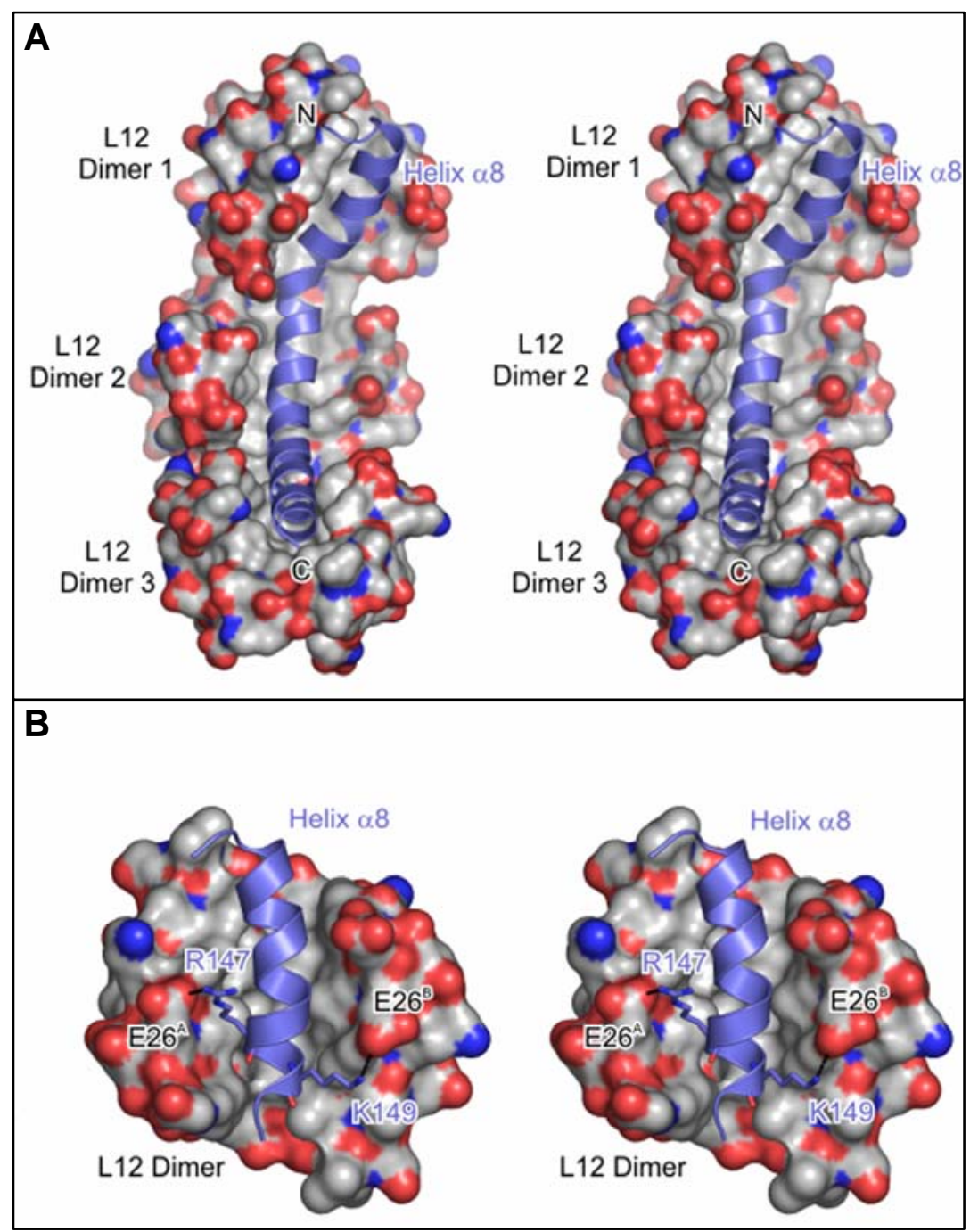

Figure 17. (A) Stereo view of the surface of the three neighboring L12 NTD dimers color-coded by atom type (carbon - gray, oxygen - red, nitrogen - blue) with the bound L10 helix $\alpha 8$ (blue ribbon). The image reveals the hydrophobic lining of the L10-binding groove (gray interior surface) and the deep burial of helix $\alpha 8$. (B) Stereo surface plot of one L12 NTD dimer, color-coded by atom type (carbon - gray, oxygen - red, nitrogen - blue) bound to a segment of L10 helix $\alpha 8$. Residues R147 and K149 of L10 engage in salt bridges with the carboxyl groups of E26 residues from the two L12 molecules (A and B) at the rim of the binding pocket.

Loops of adjacent L12 NTD dimers face each other and engage in four backboneto-backbone hydrogen bonds via residues E11, L13, V15 and S16. Turns of L10 helix $\alpha 8$, which fall at the border of two adjacent L12 NTD dimers, are pried apart by inter-dimer contacts, leading to the two kinks of helix $\alpha 8$. Inter-dimer interactions support a rigid arrangement of the three L12 NTD dimers on helix $\alpha 8$ independent of the crystal environment.

In all six L12 NTDs a universally conserved phenylalanine (F29 in T. maritima), contacts L10 in a similar manner. Specifically, all six F29 residues stack on a hydrophobic 
L10 residue: Y141, P151, I153, I162, L163, I173 (Figure 18 and Figure 22). These L10 residues delineate the borders of the three segments of helix $\alpha 8$, which carry the L12 NTD dimers. Consistently, F29 is important for the stabilization of the L12 NTD dimer onto the L10. This arrangement is in agreement with the observation that the corresponding residue of $E$. coli L12 (F30) is crucial for the interaction with L10 (Gudkov et al. 1982).

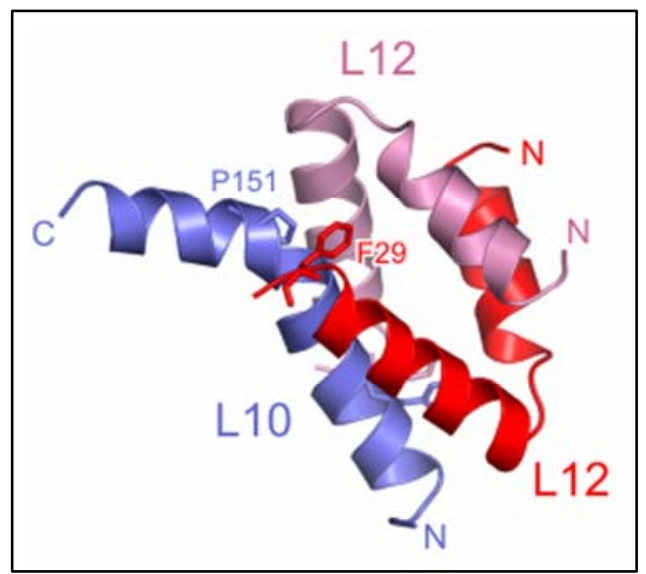

Figure 18. Diametric ribbon plots of an interaction of a tmaL10 helix $\alpha 8$ section (blue) and a tmaL12 NTD dimer (red and pink). All F29 residues of the six L12 NTD molecules stack onto hydrophobic residues (here Proline 151) from L10 helix $\alpha 8$ (interacting residues shown as sticks and labeled).

A recently characterized L12 point mutant (LL103; S15F in ecoL12, T14F in tmaL12) exhibited reduced affinity for L10 and led to reduced translational efficiency in mutant ribosomes (Nomura et al. 2003). In five of the six L12 molecules, T14 is not engaged in a direct contact to L10. Rather, its side chain hydroxyl stabilizes the tight turn between the L12 NTD helices by hydrogen bonding to the backbone nitrogen of E17. Only in the L12 molecule proximal to the L10 globular head, the T14 hydroxyl additionally engages in a hydrogen bond to the E119 carboxyl group of L10. Therefore, the present structures suggest that consequences of the LL103 mutation are mainly due to the role of T14 in the structural maintenance of the L12 NTD.

In E. coli, one L12 dimer is more tightly associated with L10 than the other (Wiggers et al. 1997). This observation agrees with the present structure where the proximal L12 NTD dimer in each of the three tmaL10:(L12NTD) ${ }_{6}$ complexes engages in interactions with the L10 NTD, which are not seen for the distal dimers. Specifically, the L12 NTD dimer proximal to the L10 NTD shows some contacts to the latter domain (e.g. $\left.\mathrm{T} 14(\mathrm{OG} 1)_{\mathrm{L} 12}-\mathrm{E} 119(\mathrm{OE} 2)_{\mathrm{L} 10} ; \mathrm{V} 15(\mathrm{~N})_{\mathrm{L} 12}-\mathrm{D} 91(\mathrm{OD} 2)_{\mathrm{L} 10} ; \mathrm{E} 20(\mathrm{OE} 1)_{\mathrm{L} 12}-\mathrm{K} 121(\mathrm{NZ})_{\mathrm{L} 10}\right)$ 
which lead to the burial of an additional $570 \AA^{2}$ of combined surface area and thus, account for a stronger adhesion compared to the other two dimers.

\section{A flexible point in L10}

In the three crystal structures, L10 helix $\alpha 8$-(L12 NTD) 6 elements adopt different orientations relative to the L10 NTD (Figure 19A). The $\alpha 8$-(L12 NTD) 6 movement can be described as rotations around a pivot point, located in the beginning of an unstructured loop that connects the L10 NTD and helix $\alpha 8$ (Figure 19A, close-up view). Different conformations observed are the result of different contacts between helix $\alpha 8$ and the L10 NTD or between the L12 NTD dimers and the L10 NTD. Figure 19B shows several sets of salt bridges formed between these domains (e.g. E119 - K137, E129 - K133), which stabilize the structure in a certain conformation. These salt bridges surround hydrophobic interactions, by which a convex surface area on the first segment of helix $\alpha 8$ bearing an L12 NTD dimer is inserted in to a concave surface area of the L10 NTD (Figure 19C). Moreover, in the R32 crystal structure derived from the tmaL10:L12 complex after in situ proteolysis, the L10 helix $\alpha 8$-(L12 NTD)6 segment adopts yet another orientation relative to the L10 NTD, further supporting the notion that this region has a certain degree of flexibility. 

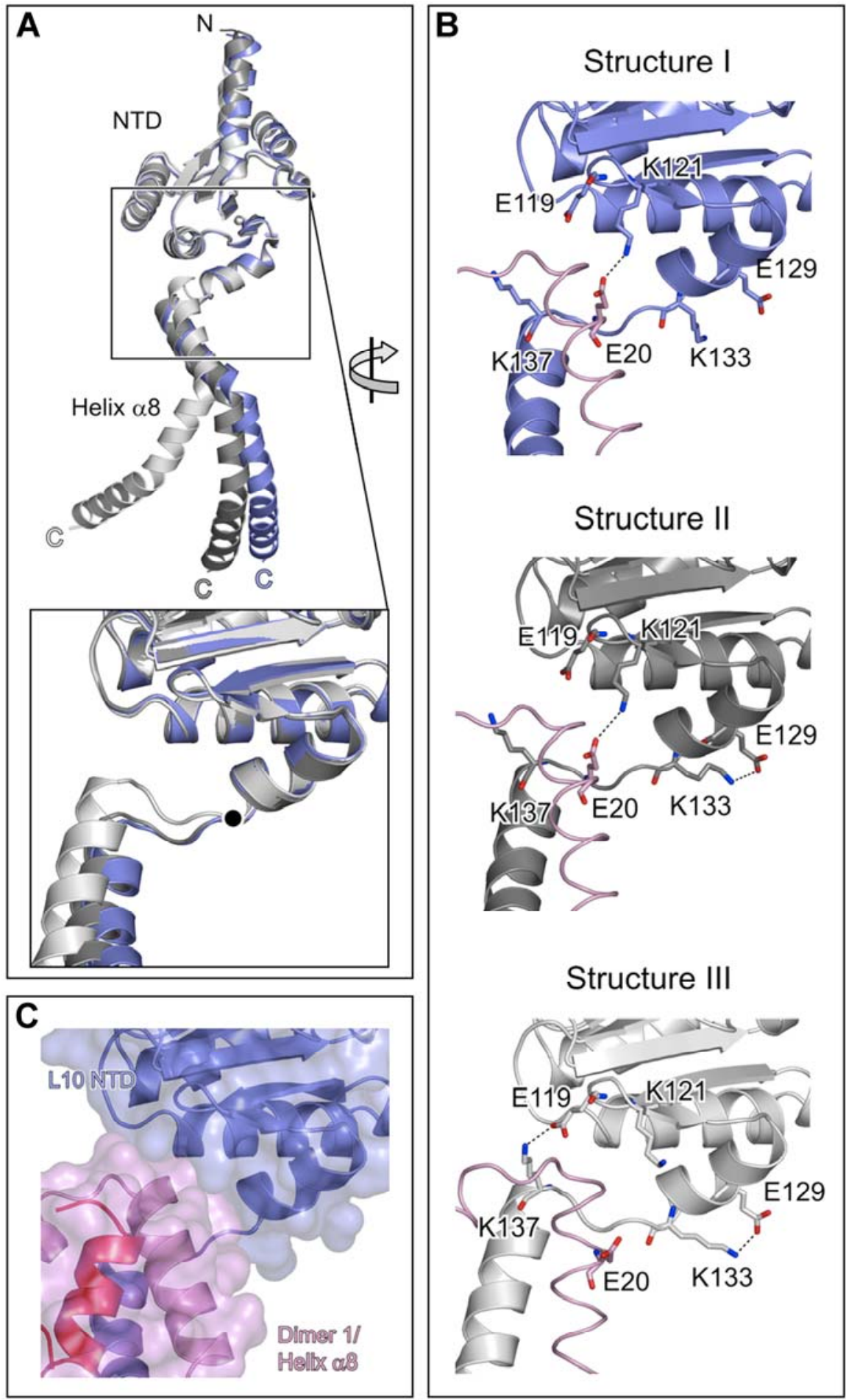

Figure 19. (A) Superposition of L10 from the three crystal structures (shown in blue, dark gray and light gray) aligned on their NTDs showing the flexible attachment of helix $\alpha 8$ to the L10 NTD. L12 NTD dimers have been omitted for clarity. In the close-up view (rotated $60^{\circ}$ clockwise about the vertical axis) the black button identifies a pivot point around which helix $\alpha 8$ rotates relative to the NTD. (B) The same view on the three individual L10 molecules as in the close-up view with the proximal L12 NTD molecule shown as a pink tube. Coloring: Carbon - colored as the L10 molecules; oxygen - red; nitrogen - blue. Dashed lines indicate salt bridges between the L10 NTD and the flexible connector or helix $\alpha 8$ and between the L10 NTD and the proximal L12 NTD molecule, which stabilize the different conformations. (C) A convex surface area of the proximal L12 NTD dimer and the N-terminal part of L10 helix $\alpha 8$ (semitransparent pink surface) inserted into a concave surface area on the L10 NTD (semitransparent blue surface). 


\section{L12 dimerization mode}

In the tmaL10:(L12 NTD) 6 structures, two L12 NTD molecules forming a dimer face each other in an antiparallel fashion (Figure 16, Figure 20).

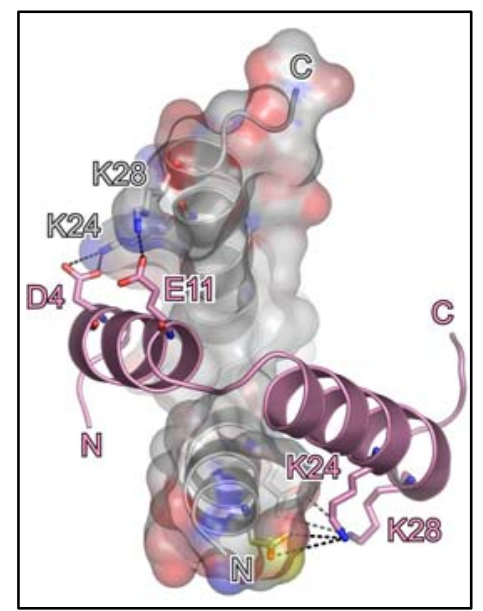

Figure 20. L12 NTD inter-dimer contacts. Stereo surface plot of one L12 NTD molecule, color-coded by atom type (carbon - gray, oxygen - red, nitrogen - blue) bound to the other NTD of L12 (pink ribbon) in an antiparallel fashion. The image reveals the prevalence of hydrophobic interactions between the two molecules (gray interior surface). Additionally, reciprocal salt bridges between Glu11 and Lys28 and between Asp4 and Lys24 are observed, strengthening inter-dimer interactions (Wahl et al. 2000a).

An identical dimerization mode (Figure 21A: between molecules I and III, as well as between molecules II and IV) was previously evidenced in the crystal structure of L12 in isolation (Wahl et al. 2000a). A second, parallel dimerization mode (Figure 21A: between molecules I and II) observed in the isolated L12 structure, is not seen in the present structures. Additionally, the N-terminal dimerization mode was previously proposed for both isolated ecoL12 and tmaL12 proteins in solution by NMR (Figure 21B; Bocharov et al. 2004) and FRET (Figure 21C; Moens et al. 2005) studies, respectively, and for ecoL12 on the ribosome (Mulder et al. 2004).

In the tmaL12 crystal structure, the hinge region of one L12 molecule folds back as an $\alpha$-helix onto two interlaced L12 NTDs (Figure 21D). In the tmaL10:(L12 NTD) 6 complex, the hinge is replaced by the ten-residue segments of L10 helix $\alpha 8$ (Figure 16, Figure 21E). This suggested that the $\alpha$-helical hinge observed in the isolated tmaL12 structure was in fact mimicking the L10 helix $\alpha 8$ of the tmaL10:(L12 NTD) 6 complex. 


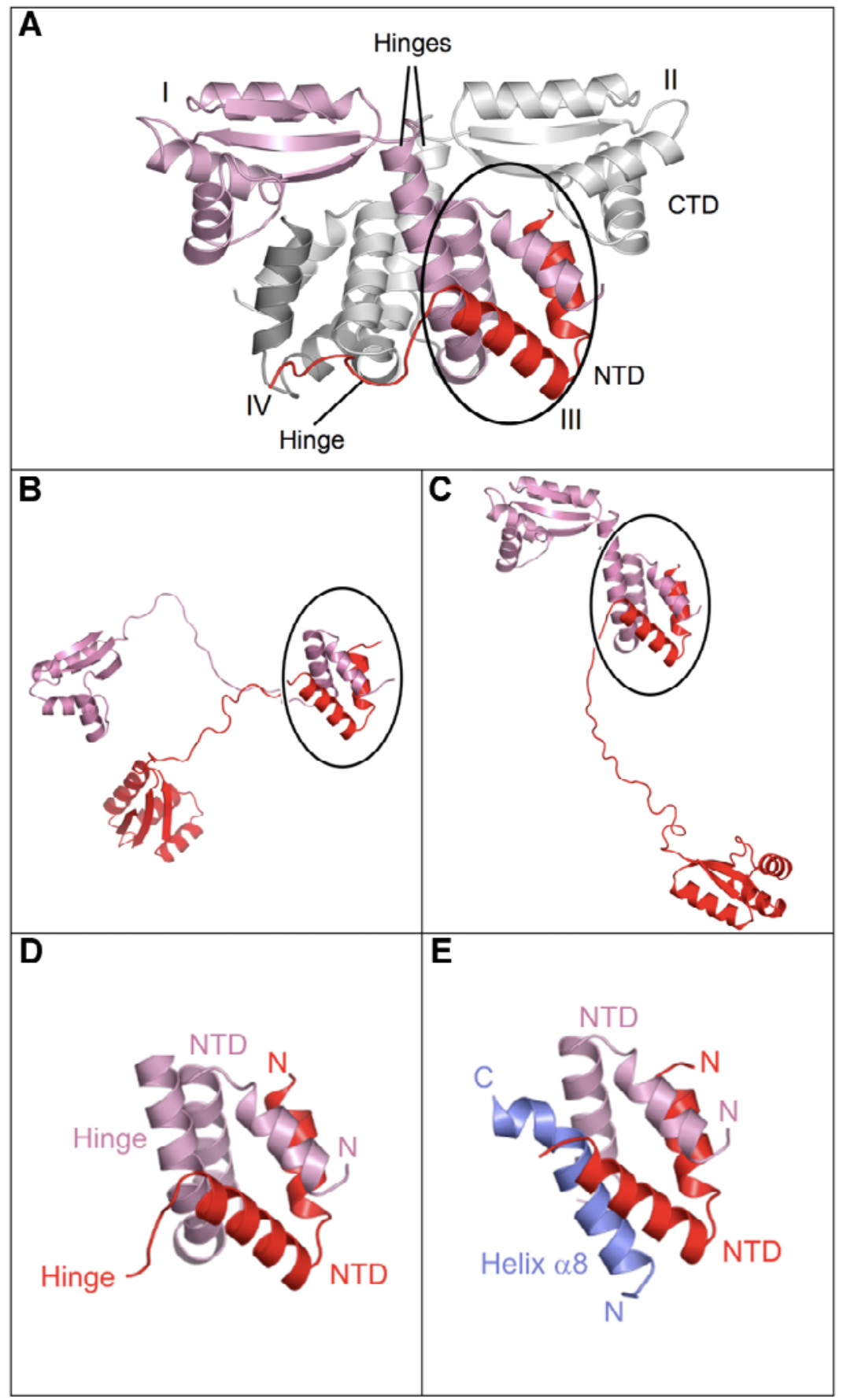

Figure 21. (A) Hetero-tetrameric arrangement in the crystal structure of isolated tmaL12 (Wahl et al. 2000a) showing one type of dimerization (parallel) between two full-length molecules (molecules I and II) and another (N-terminal) between a full-length molecule and a N-terminal fragment (I and III or II and IV). (B) ecoL12 conformation in solution: an N-terminal dimerization mode observed between L12 NTD molecules and random coil conformations of the L12 hinges (Bocharov et al. 2004). (C) Model proposed for tmaL12 conformation in solution, deducted with FRET: a N-terminal dimerization mode is seen for the L12 NTD and hinges adopt both an extended and an $\alpha$-helical conformation (Moens et al. 2005). This model is also in agreement with NMR data for ribosomal bound-ecoL12 (Mulder et al. 2004). (D) Details of the interaction between a helical hinge (pink) in complex with the NTDs (red and pink) seen in the crystal structure of tmaL12 in isolation (Wahl et al. 2000a). (E) One L12 NTD dimer (red and pink) of the present crystal structures in complex with its L10 binding region (blue). L10 helix $\alpha 8$ and the $\alpha$-helical L12 hinge of isolated L12 (the latter depicted in (D)) bind in a similar fashion to the identically structured L12 NTD dimers. 


\section{Stoichiometry of the stalk proteins}

The present crystal structures from the hyperthermophilic bacterium T. maritima revealed a 1:6 stoichiometry of the stalk proteins, namely one copy of the full-length L10 contacting six copies of L12 NTD. This stoichiometry was unexpected since in E. coli a ratio 1:4 (L10:L12) was well established (Terhorst et al. 1973; Hardy 1975; Subramanian 1975; Pettersson and Liljas 1979). However, the T. maritima L12 copy number was confirmed by several approaches: multiple sequence alignments, multi-angle laser light scattering and quantification of the ribosomal L12 by immunoblotting (the latter experiment was performed in collaboration with U. Kothe and M.V. Rodnina, Witten). Quantification of the L12 copy number by immunoblotting required functional E. coli and T. maritima ribosomes. To test the T. maritima ribosomes activity, a $70 \mathrm{~S}$ initiation complex was prepared using available $E$. coli initiation factors (IF1, IF2, IF3), mRNA (Rodnina and Wintermeyer 1995) and $\mathrm{f}\left[\mathrm{H}^{3}\right] \mathrm{Met}^{-t R N A}{ }^{\mathrm{fMet}}$. The occupancy of the P-site was $25 \%$, as determined by nitrocellulose filtration, suggesting active T. maritima ribosomes although orthologous initiation factors from E. coli were used (data not shown).

\section{a. Sequence alignment}

Sequence comparisons showed that helix $\alpha 8$ in ecoL10 is missing one of the tenresidue L12-binding sections compared to T. maritima, consistent with the notion that it can only accommodate two L12 dimers. In contrast, some other bacteria exhibit a similar length and partitioning in L10 helix $\alpha 8$ as T. maritima and are expected to maintain a L10:(L12) 6 complex (Figure 22, see Suppl. Figure 2, Appendices, for a complete denomination of all amino acids). 

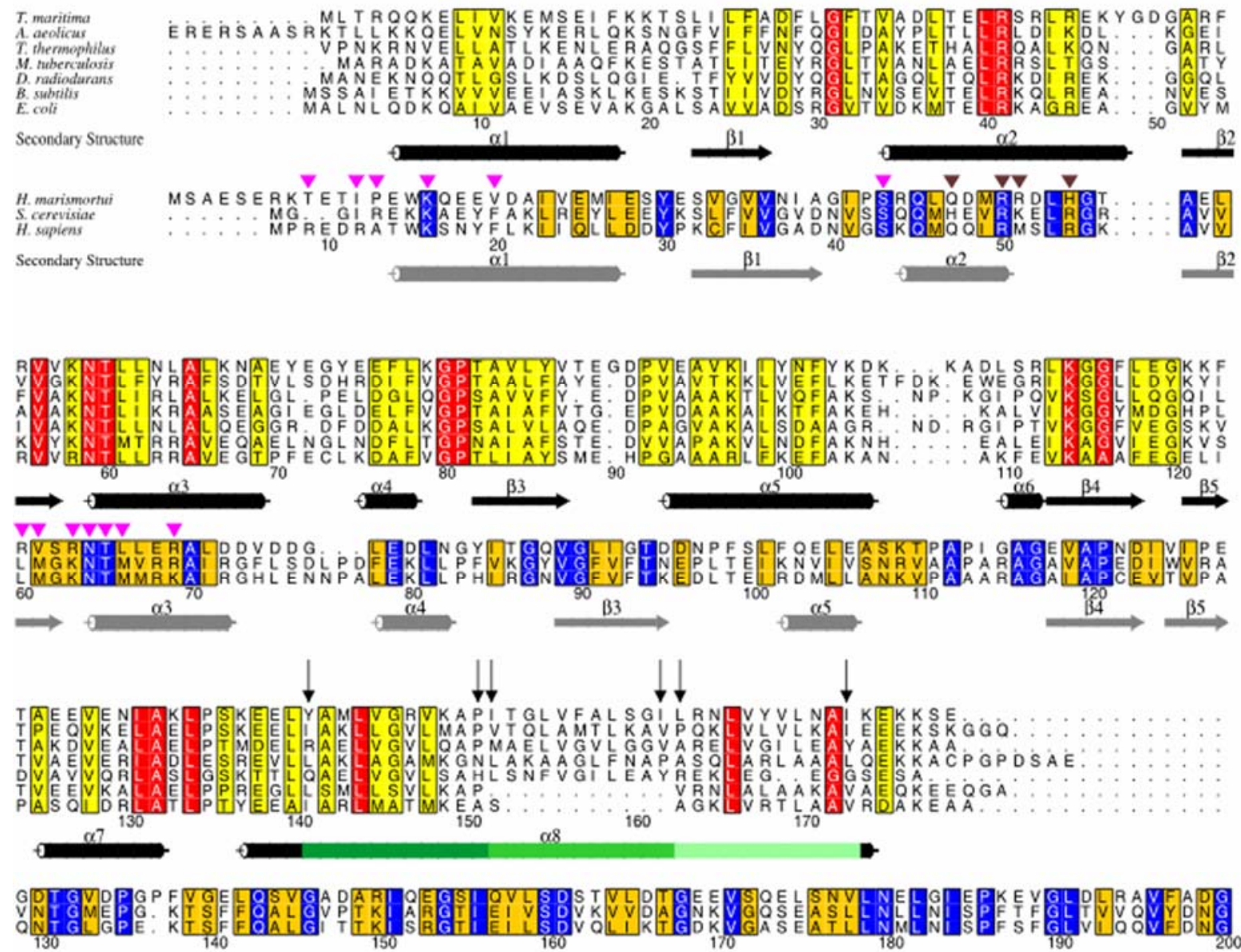

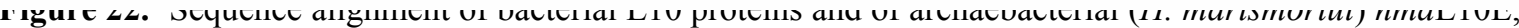
yeast P0 and human P0 proteins. Secondary structure elements of tmaL10 and hmaL10E, as revealed in the present crystal structures, are indicated below each alignment block (black and gray, respectively). Sequence numberings below the blocks correspond to tmaL10 and $h m a \mathrm{~L} 10 \mathrm{E}$, respectively. Within the bacterial L10 sequences, highly conserved amino acids are color-coded in red, intermediately conserved positions in yellow. In the L10E/P0 block, identical residues are shown in blue, conserved residues in orange. Residues of $h m a \mathrm{~L} 10 \mathrm{E}$ that interact directly with $23 \mathrm{~S}$ rRNA are labeled with a magenta triangle. Residues that contact protein L11 are labeled with a brown triangle. The three segments of helix $\alpha 8$ in tmaL10 that associate with L12 NTD dimers are indicated by different shades of green. Some bacteria (e.g. T. maritima) contain three ten-residue segments of L10, consistent with the accommodation of three L12 dimers, whereas other bacteria (e.g. E. coli) lack one ten-residue segment of L10, leading to the accommodation of only two dimers of L12. Above this segments, arrows indicate hydrophobic residues of L10 (Y141 and P152, I153 and I162, L163 and I173), which stack with the F29 side chains from the L12 NTD.

\section{b. Multi-Angle Laser Light Scattering}

In order to confirm the L12 copy number in T. maritima L10:L12 complex, both recombinant full-length tmaL10:L12 and ecoL10:L12 complexes were produced and subjected to molecular mass measurements by means of Multi-Angle Laser Light Scattering (MALLS). In addition, aaeL10:L12, tmaL10:L12 NTD, tmaL10:L12 NTD/hinge, tmaL10:ecoL12 complexes were investigated.

The production of the L10:L12 complex from E. coli, was achieved using a biscistronic plasmid (based on pGEX-5x-3 vector), with sequential genes for GST-L10 and 
L12 (provided by M.V. Rodnina, Witten). ecoL10:L12 complex was expressed in E. coli Rosetta(DE3) cells and further purified via glutathione affinity chromatography. Next, the GST tag of ecoL10 was removed by Factor Xa cleavage. Subsequent purification on both heparin and size exclusion columns yielded a highly pure complex (Figure 23A). tmaL10:L12 NTD/hinge was co-expressed after co-transformation in Rosetta(DE3) strain. The construct L12 NTD/hinge was designed by introducing a stop codon after codon 53 (corresponding to the end of the hinge region) in the same plasmid used for expression of full-length tmaL12 (Wahl et al. 2000b). The purification of the resulting complex was employed as described for tmaL10:L12 and tmaL10:L12 NTD (Figure 23B). The tmaL10:ecoL12 complex was obtained from the plasmid pETM-ZZ containing the gene for $\mathrm{His}_{6}$-L10 and the plasmid pT7-6::rplL, comprising the ecoL12 gene without any affinity tag (Oleinikov et al. 1993). Following co-transformation and co-expression, the resulting protein complex was purified by Ni-NTA affinity chromatography and size exclusion, as described for the $t m a \mathrm{~L} 10$ :(L12 NTD) $)_{6}$, with the exception of heating at $80^{\circ} \mathrm{C}$. The latter step was omitted, as the ecoL12 is not thermostable beyond $\sim 65^{\circ} \mathrm{C}$ (Figure $23 \mathrm{C}$ ). The purity of all the resulting complexes was estimated at $>90 \%$ according to Coomassie blue-stained SDS gels. 


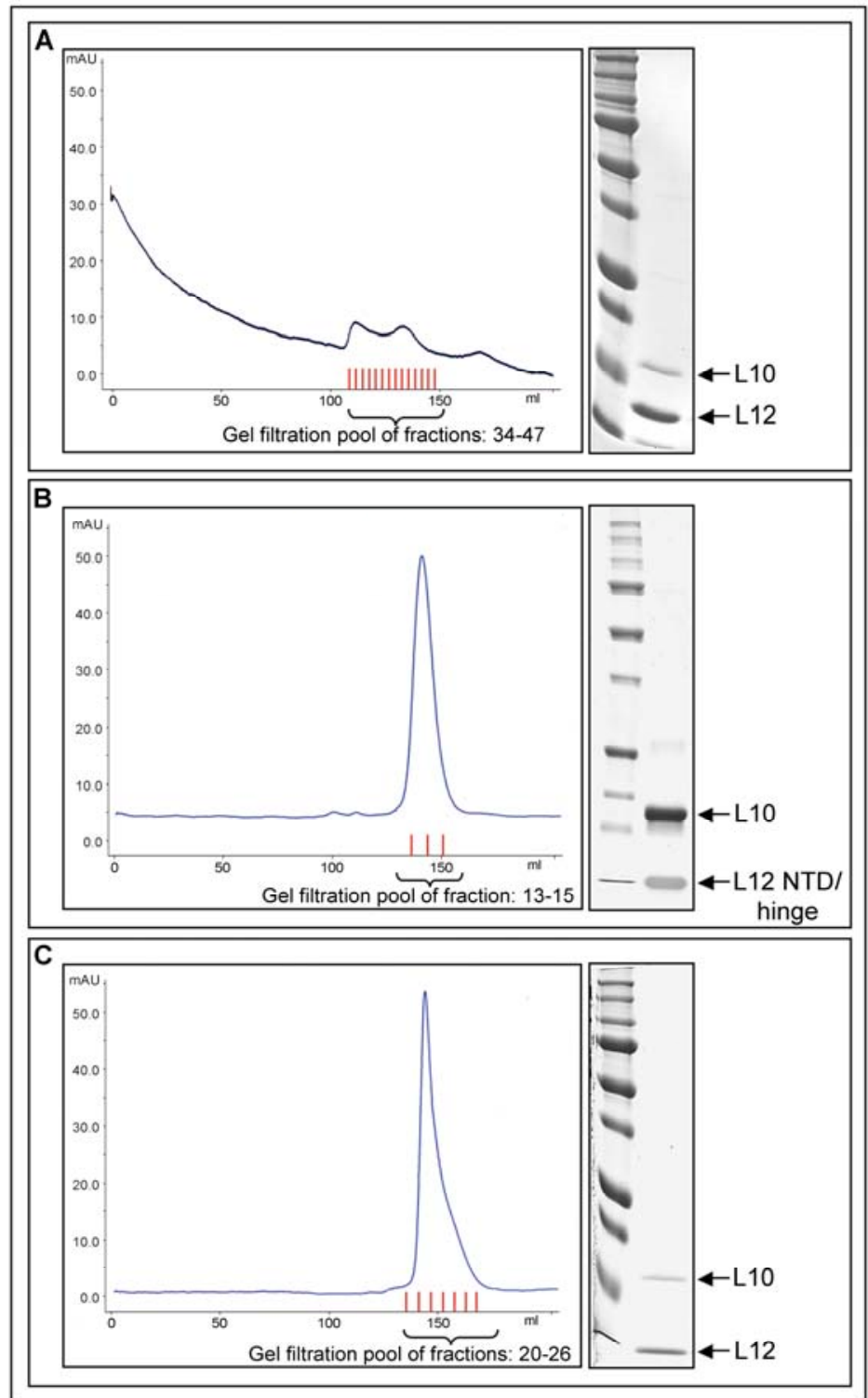

Figure 23. (A) ecoL10:L12 protein complex. Chromatogram representing the elution profile of the ecoL10:L12 complex on the Superdex 75 26/60 prep grade gel filtration column, as a last step of the purification process. Fractions 34 - 47 were pooled and concentrated to $10 \mathrm{mg} / \mathrm{ml}$. The ecoL10:L12 complex, final preparation, representing a complex between $17.6 \mathrm{kD} \mathrm{L10}$ and $12.2 \mathrm{kD} \mathrm{L12}$ proteins is also depicted. (B) $t m a \mathrm{~L} 10: \mathrm{L} 12 \mathrm{NTD} /$ hinge protein complex. Elution profile of the gel filtration, as a last step of the complex purification. Fractions $13-15$ were combined and concentrated to $10 \mathrm{mg} / \mathrm{ml}$. The final preparation, representing a complex between the $20.3 \mathrm{kD} \mathrm{L10}$ protein and the $6 \mathrm{kD} \mathrm{L12} \mathrm{NTD/hinge} \mathrm{fragments} \mathrm{is} \mathrm{shown.}$ (C) tmaL10:ecoL12 protein complex. Size exclusion chromatography of the complex, as a last step of the complex purification. Fractions containing the complex (20-26) were further concentrated to $10 \mathrm{mg} / \mathrm{ml}$. The final preparation representing a complex between the $20.3 \mathrm{kD}$ tmaL10 and the $12.2 \mathrm{kD} e c o \mathrm{~L} 12$ proteins is depicted.

Purified proteins were subsequently analyzed by multi-angle laser light scattering and refractive index detection to measure their molecular weight. All measurements were performed at room temperature. An equal amount of protein (concentration of $2 \mathrm{mg} / \mathrm{ml}$ ) was used for all the measurements. The mobile phase of the system was PBS, pH 7.4. 
Ultra pure BSA was used as a control. Shortly after its solubilization, this protein forms monomers and, to a lesser extent, dimers and trimers, which were identified by MALLS measurement as three different peaks (marked in Figure 24A, and clearly distinguished by plotting molar mass versus volume in Figure 25A).
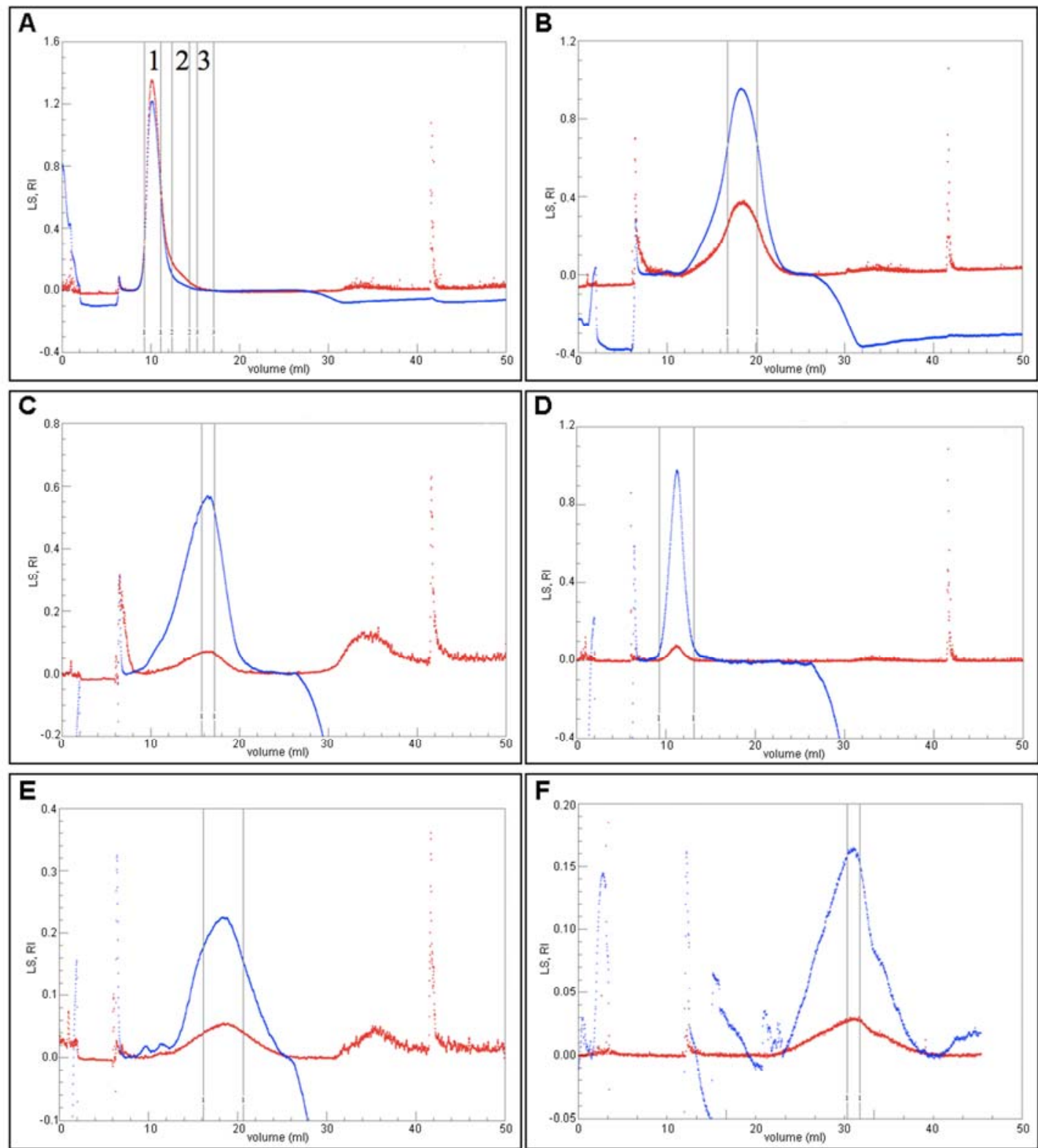

Figure 24. Light Scattering (LS: signal of the $90^{\circ}$ light scattering detector, red curve) and Refractive Index (RI, blue curve) values of BSA or L10:L12 complexes are normalized and plotted versus volume [ml] in a flow field-flow fractionation chromatography. The peak area selected for mass measurement is delimited by parallel lines. (A) BSA elution profile. A major peak corresponds to the monomer state of the protein. At the shoulder of this peak, two additional peaks correspond to dimer and trimer states, respectively; the latter two peaks are too small to be distinguished in this graphic, however, the molar masses corresponding to these peaks are clearly evidenced in a plot of molar mass versus volume in Figure 25A. (B-F) Elution profiles of L10:L12 complexes from several bacteria. All the complexes eluted as single peaks. (B) tmaL10:(L12) ${ }_{6}$; (C) tmaL10:(L12 NTD)6; (D) tmaL10:(L12 NTD/hinge) 6 ; (E) aaeL10:(L12)6; (F) ecoL10:(L12) 4 . 
All the L10:L12 complexes eluted as single peaks (Figure 24B-F, Figure 25A). Molar mass determinations of L10:L12 complexes are summarized in Table 13. Multiangle laser light scattering indicated a molecular mass of $101.3( \pm 1.5) \mathrm{kD}$ for the tmaL10:(L12) 6 complex, in excellent agreement with the predicted mass of $102.6 \mathrm{kD}$ for a heptametric complex (Figure 25A). A recombinant full-length ecoL10:(L12) ${ }_{4}$ complex showed a mass of $69.7( \pm 2.3) \mathrm{kD}$ as compared to $66.3 \mathrm{kD}$ calculated for a pentameric composition (Figure 25A). The latter result is in line with the previously reported L12 copy number on the ribosome. Moreover, data obtained for tmaL10:L12 and ecoL10:L12 complexes were similar to those obtained from the quantification of the L12 copy number on E. coli and T. maritima ribosomes by immunoblotting (U. Kothe, M.V. Rodnina, Witten).

\begin{tabular}{lccccc}
\hline \hline & & & \multicolumn{2}{c}{ L10:L12 Stoichiometry } \\
\multicolumn{1}{c}{ Protein Complex } & Theor. Mass (kD) & Exp. Mass (kD) & MALLS & Crystal & Radioact. \\
\hline \hline tmaL10:L12 & 102.6 & $101.3 \pm 1.5$ & $1: 6$ & & \\
tmaL10:L12 NTD & 40.9 & ${ }^{\mathrm{b}} 44.6 \pm 17$ & $1: 6$ & $1: 6$ & \\
tma L10:L12 NTD/hinge & 56.3 & $54.6 \pm 7$ & $1: 6$ & & \\
a $\operatorname{ae}$ L10:L12 & 103.9 & ${ }^{\mathrm{b}} 102.3 \pm 15$ & $1: 6$ & & \\
ecoL10:L12 & ${ }^{\mathrm{a}} 66.3$ & $69.7 \pm 2.3$ & $1: 4$ & & ${ }^{\mathrm{c}} 1: 4$ \\
\hline \hline
\end{tabular}

Table 13. Size determination of L10:L12 complexes from different bacteria. ${ }^{\text {a }}$ The ecoL10:(L12) $)_{4}$ molar mass, was previously determined by mass spectrometry as $66.3 \mathrm{kD}$ (Hanson et al. 2003). ${ }^{\mathbf{b}}$ The molar mass was calculated applying the smoothing option (excluding extreme molar mass values) of the program used for data analysis. Initial values of the tmaL10:L12 NTD and aaeL10:L12 were 54 and $117 \mathrm{kD}$, respectively. 'Quantification of the L12 copy number on the E. coli ribosome using labeled amino acids (Subramanian 1975).

Additionally, both tmaL10:L12 NTD and tmaL10:L12 NTD/hinge size determinations confirmed the occurrence of the heptametric complex in T. maritima, exhibiting experimental molar masses close to the predicted values (Figure 25A). The tmaL10/ecoL12 molar mass could not be determined, despite several buffer, protein concentration and system parameters variations. The mass measurement suggested a potential aggregation of this complex. However, a peculiar conformation of this hybrid complex in the tested solutions could also have impaired the proper mass determination by means of MALLS. Interestingly, aaeL10:L12 yielded a molar mass corresponding to a heptametric complex (Figure 25A). This result suggested that in some bacteria (presumably comprising all thermophilic bacteria) the L10:L12 complex exhibits a 1:6 stoichiometry (Table 13). Supporting evidence came from a recent report, which identified 
by tandem mass spectrometry the occurrence of L10:(L12) 6 complexes on ribosomes of thermophilic bacteria (Ilag et al. 2005).

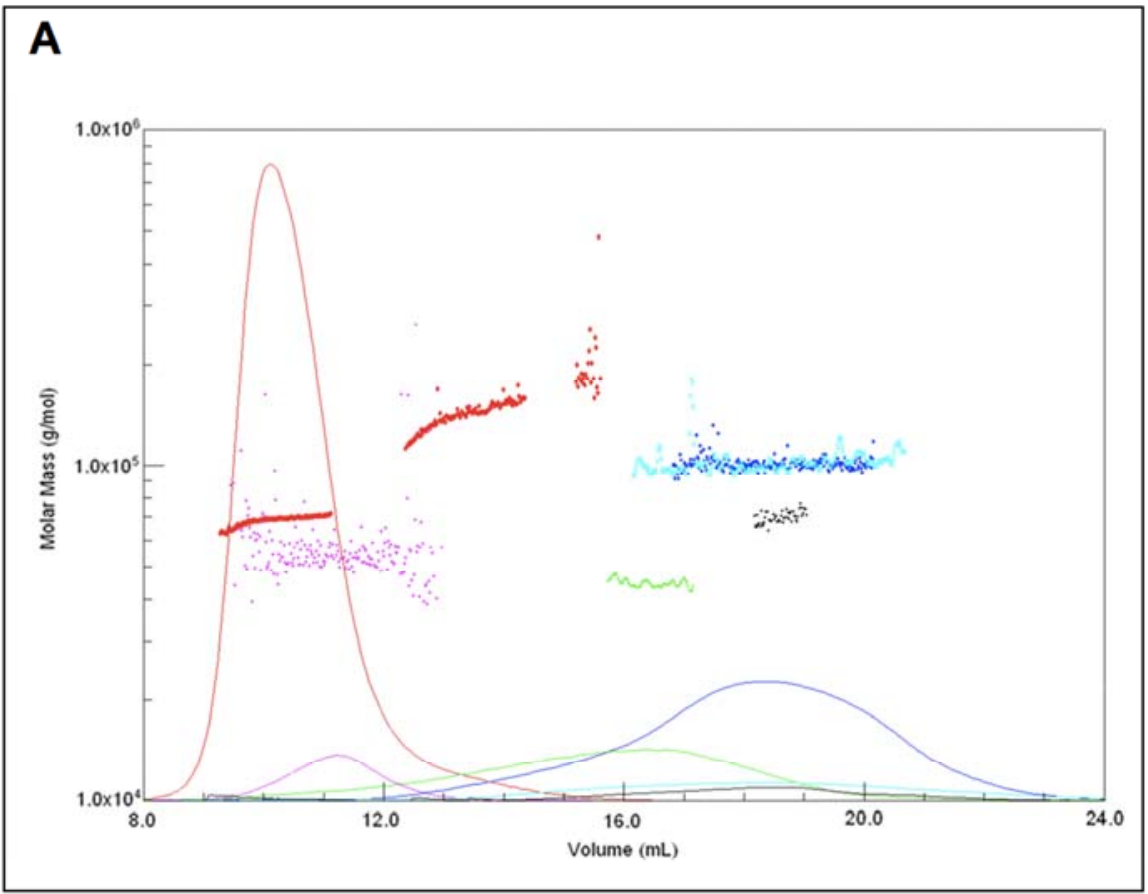

\section{B}

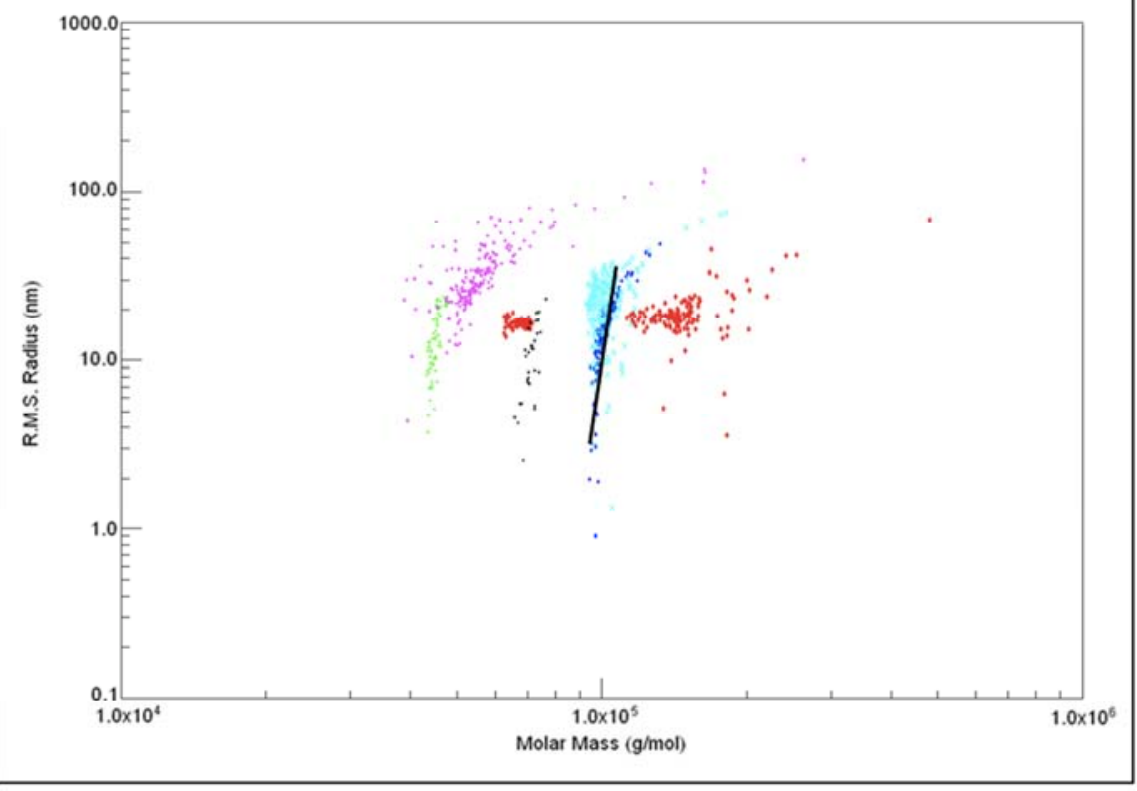

Figure 25. (A) Distribution of the Molar Mass [g/mol] vs. Volume [ml] of BSA and L10:L12 complexes from several bacteria. BSA (red): with a calculated mass of $68.69 \pm 1.6$ (67) for the monomer, $136.3 \pm 12$ (134) for the dimer and 198.4 \pm 77 (201) for the trimer states, respectively (the theoretical mass is shown in parentheses); tmaL10:(L12) (blue); tmaL10:(L12 NTD) 6 , (green); tmaL10:(L12 NTD/hinge) ${ }_{6}$ (pink); aaeL10:(L12) 6 (cyan); ecoL10:(L12) 4 (black). (B) Root Mean Square Radius [nm] vs. Molar Mass [g/mol] of BSA and L10:L12 complexes from several bacteria (color code as in (A)). One slope (black line) is drawn for the distributions of the RMS Radius $v s$. Molar Mass of the tmaL10:(L12) 6 (blue), suggesting that no conformational changes occurred for this protein in solution. 
By plotting the root mean square (RMS) radius versus molar mass of BSA and L10:L12 complexes (Figure 25B), it was possible to observe potential conformational changes in solution of the analyzed samples. A slope (Figure 25B, black line, exemplified for tmaL10:(L12) 6 ) could be drawn for each of the distributions of RMS radius versus molar mass. The existence of a single slope for each of the samples instead of, e.g. two or more slopes separated by transition regions, suggests that there are no conformational changes in solution for either BSA or L10:L12 complexes. The steepness of the slope can easily be related to the conformation of the molecules, with extended structures giving larger slopes than compact molecules. As expected, monomer-state BSA is less extended than the dimer- and trimer-states. The least extended L10:L12 complexes seem to be ecoL10:(L12) 4 and tmaL10:(L12 NTD) 6 , as compared to the tmaL10:(L12 NTD/hinge) 6 , tmaL10:(L12) ${ }_{6}$ and aaeL10:(L12) ${ }_{6}$ complexes.

Taken together, the multi-angle laser light scattering mass determinations were in agreement with the crystal structures showing a tmaL10:(L12 NTD) 6 organization. Additionally, these results reconfirmed that in E. coli four copies of L12 can be accommodated by one L10 protein. Both T. maritima and E. coli L10 share a high degree of sequence conservation (approximately 65\% identity (Wahl et al. 2000b)). Therefore it can be suggested that E. coli and T. maritima stalk proteins display a similar organization. The only difference is the absence of one of the three repetitive elements L10 helix $\alpha 8$-L12 NTD in E. coli, resulting in the accommodation of only two dimers of L12. Thus, it can be concluded that the length of L10 helix $\alpha 8$ determines the number of L12 copies per ribosome. The increased copy number of L12 found in some bacterial ribosomes emphasizes the importance of multiple L12 copies for the mechanism of translation. 


\section{$\underline{\text { Discussion }}$}

\section{The tmaL10:(L12 NTD) 6 complex}

The crystal structure of the bacterial L10:(L12 NTD) $)_{6}$ complex determined herein reconciles numerous previous structural and biochemical data. The present structures of L12 NTD molecules in complex with L10 are consistent with the observation that the Nterminal part of L12 is responsible for dimerization (Gudkov and Behlke 1978). The Nterminal dimerization mode of the L12 NTDs observed herein is in agreement with one of the dimerization modes found in the isolated tmaL12 crystal structure (Wahl et al. 2000a). This dimerization mode was also described in ecoL12 in solution (Bocharov et al. 2004) and tmaL12 in solution (Moens et al. 2005). However, a compact, parallel dimerization mode in the tmaL12 crystal structure, which was mediated by two helical hinges (Wahl et al. 2000a), is not seen in L10:(L12 NTD) 6 complexes. Indeed, the formation of such a dimer is obstructed in the tmaL10:(L12 NTD) 6 complexes by the binding of L10 helix $\alpha 8$ to the L12 NTD dimers at a position, which in the compact L12 dimer is occupied by a helical L12 hinge region. Displaced from their binding scaffold by L10 helix $\alpha 8$, the L12 hinges are likely to be unstructured, as seen for ecoL12 in solution (Bocharov et al. 2004; Mulder et al. 2004). Thus, the compact, parallel dimerization via helical L12 hinge regions is of no relevance to the ribosome-bound form of L12. These results refute a tentative model of the stalk in a 70S ribosome crystal structure (Yusupov et al. 2001), which was based on this compact L12 dimer structure.

In the present tmaL10:(L12 NTD) 6 crystal structures, three L12 NTD dimers are found to bind to sequential segments on the C-terminal helix $\alpha 8$ of L10. By deleting 20 to $33 \mathrm{C}$ terminal residues of ecoL10, the binding of both ecoL12 dimers was abolished, while the deletion of the terminal ten residues led to the loss of a single dimer (Griaznova and Traut 2000). An alignment of ecoL10 with tmaL10 (Figure 22) suggests that deletion of the terminal ten residues removes about one third of the predicted C-terminal L12 dimer binding region of ecoL10 helix $\alpha 8$. This deletion leaves the proximal L12 dimer binding site of helix $\alpha 8$ intact, accounting for the loss of a single dimer. Deleting between 20 and 33 residues, which are predicted to encompass the entire helix $\alpha 8$ in ecoL10, accounts for the loss of both L12 dimers from the complex. These results, in conjunction with the 
present crystal structures, underscore the modular design of the helix $\alpha 8$-L12 binding region, which consists of two (E. coli) or three (T. maritima) repetitive L12 NTD dimerhelix $\alpha 8$ elements. Notably, while the C-terminal deletion mutants of L10 lose their ability to bind L12, they remain unaffected in their binding activity to the ribosome (Griaznova and Traut 2000). These data are entirely in line with the assignment of the L10 NTD as a separate folding unit, which is necessary and sufficient for the interaction with the $23 \mathrm{~S}$ rRNA. Furthermore, the analyses of ecoL10 deletion mutants, which are in excellent agreement with the tmaL10:(L12 NTD) 6 crystal structures, provide strong evidence that the fundamental structural principles in the L10:L12 complexes are conserved throughout the bacterial kingdom and that differences, e.g. between E. coli and T. maritima, are purely quantitative in nature (one additional modular element in the peripheral stalk region), but has most likely no fundamental functional consequences. The latter conclusion is corroborated by the fact that effects of a large number of mutations in the ecoL10:L12 complex can be explained by the present crystal structures from T. maritima. F30 of ecoL12 (F29 of tmaL12) has been suggested to be crucial for the interaction with L10 (Gudkov et al. 1982). This hypothesis was confirmed by the crystal structure since in all six L12 molecules, F29 stacks on a hydrophobic L10 residue, which delineates the borders of helix $\alpha 8$ segments (Figure 18, Figure 22). Another point mutation S15F in ecoL12 (T14F in tmaL12) was shown to reduce the translational efficiency due to a reduced affinity for L10 (Nomura et al. 2003). However, the present crystal structure revealed that T14 is not engaged, as expected, in a direct contact to L10, and the effects of this mutation are most likely due to the role of this amino acid in the structural maintenance of the L12 NTD. Additionally, the crystal structure offers an explanation for the suggestion that in $E$. coli, one of the L12 dimers is more tightly bound to L10 than the other (Wiggers et al. 1997). Indeed, the proximal dimer of L12 in the tmaL10:(L12 NTD) 6 structure engages in interactions with the L10 NTD, which are not observed for the other two dimers.

The localization of the tmaL10:(L12 NTD) 6 on the large ribosomal subunit (Diaconu et al. 2005) revealed an extensive set of interactions between L10 NTD and 23S rRNA and only a small number of contacts to protein L11, which binds to the 23S rRNA in close vicinity to L10. Both L10 and L11 were previously shown to bind cooperatively to the ribosome (Dijk et al. 1979; Beauclerk et al. 1984; Egebjerg et al. 1990). However, the small number of direct interactions between L10 and L11 are not essential for L10 binding, because 
L10:L12 complexes can bind to 23S rRNA also in the absence of L11 (Rosendahl and Douthwaite 1993). Therefore, the cooperative binding must be primarily an indirect effect. It is possible that the L10 NTD and L11 each stabilize the overall structure of the L10/L11 binding region of the $23 \mathrm{~S}$ rRNA, thus facilitating a subsequent binding of the other protein. This notion is consistent with the observation that the L10 NTD presumably recognizes primarily the conserved fold but not the exact sequence of the rRNA.

\section{Beyond the L10:(L12 NTD) 6 crystal structure}

In the present study, we determined crystal structures of an isolated bacterial L10:(L12 NTD) ${ }_{6}$ complex. However, the important issue of how this complex is anchored to the $50 \mathrm{~S}$ ribosomal subunit, remained to be addressed. Thus, combining the results of the L10:(L12 NTD) 6 crystal structure with cryo-electron microscopy and molecular model building, it was possible to elucidate the atomic structure of the L7/L12 stalk on the ribosome; additionally, rapid kinetic experiments were used to probe its factor-related functions (Diaconu et al. 2005). The data outlined in the following represented the result of a collaborative work with four laboratories.

\section{A. Placement of the tmaL10:(L12 NTD) $)_{6}$ structure on the 50S ribosomal subunit}

A density modification procedure and a published diffraction data set of the $H$. marismortui 50S subunit (PDB accession code 1S72 (Klein et al. 2004); Suppl. Figure 3A, Appendices) allowed the tracing of the complete NTD of archaeal L10 ( $h m a \mathrm{~L} 10 \mathrm{E})$ within the electron density of the large ribosomal subunit (Frank Schlünzen and Jörg M. Harms, Max-Planck Research Group, DESY, Hamburg). L10E NTD was found to engage in extensive contacts with the thiostrepton loop and in a small number of interactions with the neighboring CTD of protein L11E (Suppl. Figure 3B, Appendices). Interestingly, despite a lack of significant sequence identity, the superposition of the bacterial L10 NTD and archaeal L10E NTD, revealed a very similar overall fold. A similar observation was made for the other elements forming the base of the stalk (L11, L10/L11 binding region of rRNA).

These findings pointed to the notion that bacterial and archaeal L10 NTD interact in a similar fashion with the L10/L11 binding region of rRNA. Thus, the tmaL10 NTD 
could be placed on the 50S subunit. The fitting of this domain was further used to line up the entire L10:(L12 NTD) 6 structure (Suppl. Figure 3C, Appendices) on the ribosome (Suppl. Figure 3D, Appendices).

\section{B. Cryo-EM reconstructions of $\mathrm{L} 7 / \mathrm{L} 12$ stalk elements}

The reconstructed model of the stalk may account for the extended features previously observed in several cryo-EM maps of ribosomes in different phases of translation (Figure 6C). To prove this hypothesis, the resulting model of the stalk was positioned into a cryo-EM envelop of an E. coli 70S:EF-G:GDP:fusidic acid (Suppl. Figure 4, left, Appendices) and into various EM maps of ribosome-factor complexes available from public databases (performed by Niels Fischer and Holger Stark, Research Group of 3D Electron Cryo-Microscopy, MPI for Biophysical Chemistry, Göttingen). Convincing fits to a well-defined, extended density neighboring the thiostrepton loop were achieved by shortening the L10:(L12 NTD) 6 complex as envisioned for E. coli stalk and through a slight rotation of the L10 C-terminal $\alpha$-helix in the flexible connector to its NTD (Suppl. Figure 4, right, Appendices). The L12 hinges and CTDs could not be located in the density of the E. coli 70S:EF-G:GDP:fusidic acid complex, probably due to their mobility.

\section{Active sites of the $\mathrm{L} 7 / \mathrm{L} 12$ stalk and their factor-related functions}

The L7/L12 stalk architecture displays an arrangement with three flexible regions: (i) the connection between the stalk base and the bulk of the 23S rRNA; (ii) the flexible region in L10; (iii) the highly mobile L12 hinges. This distribution of flexible regions suggests that the mobility progresses towards the extremity of the stalk. This prompted the hypothesis that L12 CTDs carried by highly flexible hinges might represent the active sites of the stalk, responsible for translation factor-related functions. To ascertain this notion, ribosomes lacking or containing mutant L12 CTDs were generated. By a specific ethanol/salt treatment, wild-type ribosomes were depleted of L12 and subsequently replaced with L12 that entirely lacked CTDs or exhibiting mutations in conserved surface residues of the CTDs. Using fast kinetic measurements it was then possible to differentiate effects on initial factor binding and on stimulation of GTP hydrolysis (Ute Kothe, Marina 
V. Rodnina, Physical Biochemistry Department, University of Witten/Herdecke; A. Tonevitsky, Biological Department, MV Lomonosov Moscow State University). Ribosomes depleted in L12 CTD exhibited a decrease in the initial factor binding by more than one order of magnitude. In addition, their removal was found to induce a diminution of the rate of GTP hydrolysis on the ribosome by EF-Tu or EF-G of approximately 1000 times. The effects of removing the CTDs were comparable to cores depleted of entire L12 molecules. These results pinpointed L12 CTDs as active sites for initial factor binding and strong stimulators of GTPase reaction within the stalk. To identify the amino acids responsible for GTPase activation, point mutations of all conserved surface residues of L12 CTD were investigated by similar procedures. However, none of the point mutants abolished the GTPase activation, suggesting that none of the amino acid side chains of L12 is involved in catalysis. To further elucidate the stalk function in factor recruitment, the exchange between the ribosome-bound and factor-free L12 both in the presence and absence of EF-G was examined. Unlike its eukaryotic counterparts who appear to perform this exchange, bacterial L12 are found prevalently bound to the ribosome. Only a slow exchange between the free and ribosome-bound bacterial L12 was observed, independent of the presence or absence of EF-G. These findings disfavor models suggesting the recruitment of translation factors to the ribosome through the free L12 pool in bacteria.

\section{The L7/L12 stalk: structural model and function in translation}

\section{A. Structural organization of the L7/L12 stalk}

The placement of the tmaL10:(L12 NTD) 6 crystal structure on the 50S subunit, subsequent localization of a L10:(L12 NTD) $)_{4}$ element in cryo-EM reconstructions of $E$. coli $70 \mathrm{~S}$ ribosomes, and additional guidelines provided by structures of tmaL11-rRNA complex (Wimberly et al. 1999) and ecoL12 in solution (Bocharov et al. 2004), allowed the devising of a complete atomic model of the L7/L12 stalk (Figure 26).

The stalk was divided into three structural and functional segments, delimited by flexible regions: (i) the stalk base comprising the entire L10/L11 binding region of $23 \mathrm{~S}$ rRNA, L11 and the L10 NTD; it serves as an attachment site for the peripheral components of the stalk, positioning them in the neighborhood of the ribosomal factor 
binding site; (ii) the C-terminal $\alpha$-helix of L10 in complex with the L12 NTDs that is flexibly attached to the stalk base, as seen in the three different crystal structures of tmaL10:(L12 NTD) 6 and in the EM analysis; it can therefore be regarded as a moveable platform that carries remaining elements of L12 protein; (iii) highly mobile $\underline{L 12}$ CTDs that are attached to the mobile platform via the hinge regions; most likely the L12 hinges predominantly adopt random coil structures, as in isolated L12 (Bocharov et al. 2004), because they are displaced from the L12 NTD dimers by L10 helix $\alpha 8$, in agreement with recent NMR data on 70S ribosomes (Mulder et al. 2004). Additional evidence supporting the intrinsic flexibility of the L12 hinges derives from the R32 crystal structure of the L10:L12 complex from T. maritima. Attempts to solve the entire structure failed due to an unstructured region in L12 corresponding to the hinge domains.

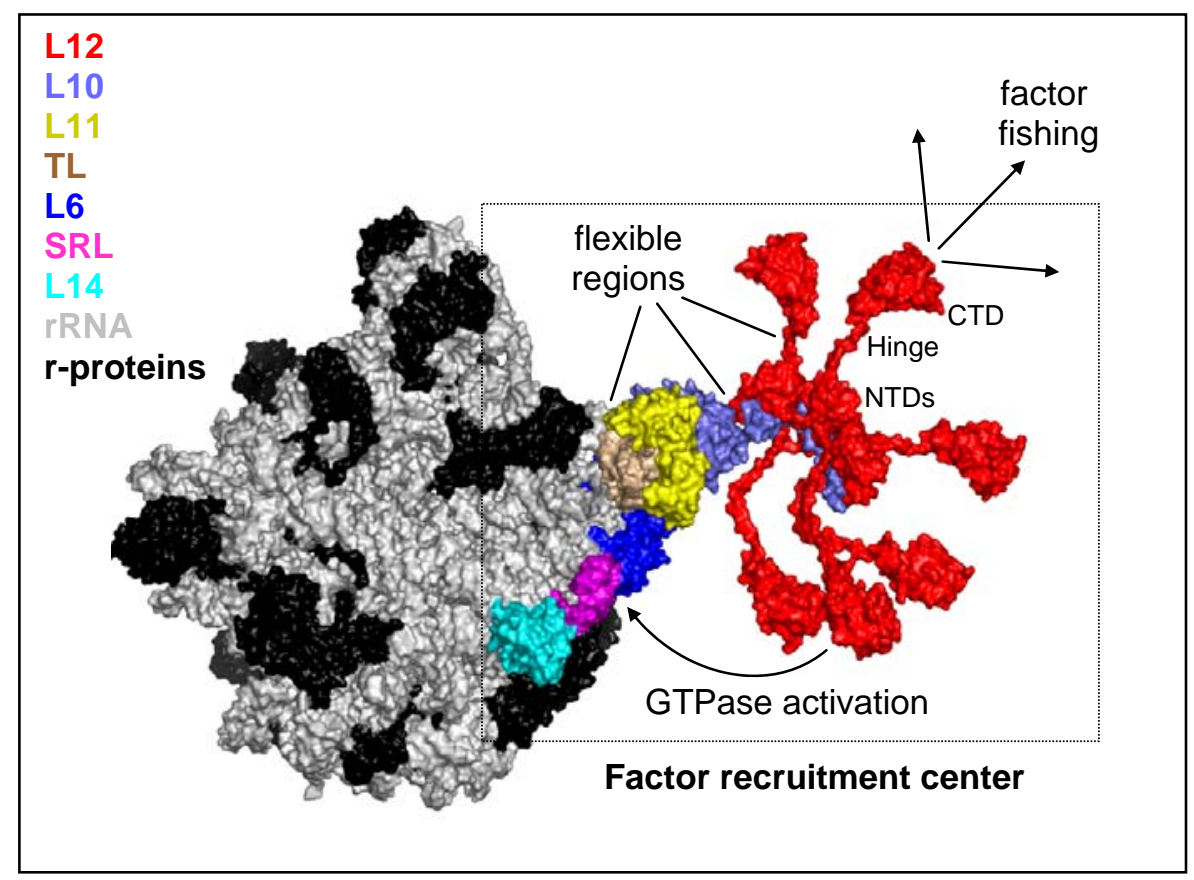

Figure 26. A complete structural model of the L7/L12 stalk comprising a L10:(L12)6 complex on the $50 \mathrm{~S}$ subunit. Proteins and rRNA regions are color-coded in the legend on the left.

Two flexible regions separate the stalk elements: the unstructured loop that connects the L10 NTD to helix $\alpha 8$ and the flexible hinge region intervening between the NTDs and CTDs of L12 and providing a high freedom of motion for the L12 CTDs. As documented by the above mentioned kinetic analysis, the L12 CTDs perform the functional interactions with the factors, and thus constitute the 'active sites' of the stalk. Restriction of L12 CTD mobility by hinge deletions inactivates the ribosome (Gudkov et 
al. 1991; Oleinikov et al. 1993), indicating that the high degree of freedom of the L12 CTDs is crucial for the activity of the stalk.

\section{B. Dynamics of the stalk during translation}

Cryo-EM analysis showed that the extended structural elements of the stalk neighboring the base undergo large rearrangements during translation (Agrawal et al. 1999) (Figure 6B, C). Previously, these mobile stalk elements were attributed to the hinge regions and CTDs of one L12 dimer (Dey et al. 1998). The present results suggest that the extended stalk elements revealed by cryo-EM rather represent the L10 helix $\alpha 8$-L12 NTD portion of the stalk. According to the proposed model (Figure 26), L10 helix a8-L12 NTD and the L12 CTDs can move relative to one another and relative to the stalk base (L10/L11-binding region of 23S rRNA, L11, L10 NTD). Cryo-EM reconstructions identified different preferred locations of the L10 helix $\alpha 8$-L12 NTD part with respect to the stalk base, in 70S:EF-G:GDP:fusidic acid experimental density map and in numerous other EM structures form the database (e.g. 70S:RF2 complexes (data not shown) (Rawat et al. 2003)). Thus, EM provides suitable restrains to position portions of the present crystal structures reliably on the 50S subunit. Differences in the orientation of the helix $\alpha 8$-(L12 NTD) ${ }_{4}$ extension seen in complexes with EF-G and RF2 point that its position changes during translation. However, the electron density vanishes beyond helix $\alpha 8$-(L12 NTD) 4 suggesting that remaining parts of L12 (hinges and CTDs) adopt multiple orientations with respect to the helix $\alpha 8-(\mathrm{L} 12 \mathrm{NTD})_{4}$ segment and apparently are too mobile to be located.

In the EM structure of a 70S:EF-G complex in the GTP conformation (Agrawal et al. 1999), a bifurcated protrusion is visible at a similar position as in the 70S:EF:G:GDPfusidic acid and 70S:RF2 complexes (Figure 6B). The bifurcated parts appear slimmer than the extended density seen in the 70S:EF-G:GDP:fusidic acid and 70S:RF2 structures but seem to have comparable lengths. One possibility is that they correspond to two populations of the helix $\alpha 8-(\mathrm{L} 12 \mathrm{NTD})_{4}$ structure, which are not fully occupied. This structure could not be interpreted in more detail, since the EM density was not available from public databases at the time of this work. However, the mobility of the helix $\alpha 8$-(L12 NTD) 4 part relative to the L10 NTD may be more extensive than suggested by the crystal structures, underlining its possible role in positioning L12 hinges and CTDs. 
Two possibilities could be envisioned regarding the stabilization of the L10 helix $\alpha 8$-L12 NTD part in certain states. First, it has been proposed that upon binding of EF-G and during GTP hydrolysis the L11 NTD moves out independently of the remainder of the stalk base due to direct contacts to the factor (Agrawal et al. 2001). Our structures show that the L11 CTD maintains interactions to the L10 NTD. Thus, L11 may constitute a bridge between EF-G and L10 NTD. It is therefore conceivable that structural changes in L11 are communicated to the L10 NTD and could favor a particular interaction between the L10 NTD and the proximal L10 helix $\alpha 8$-L12 NTD dimer element. Examples for three different interaction modes between the latter two components can be seen in the different crystal structures of the L10:(L12 NTD) 6 complex (Figure 19B, C). Second, direct L12 CTD-ribosome or CTD-factor contacts could form in certain functional states, thereby restricting the mobility of L10 helix $\alpha 8$-L12 NTD. The possibility of direct L12 CTDribosome interactions has previously been suggested by crosslinking (Dey et al. 1998), NMR (Mulder et al. 2004) and EM studies (Montesano-Roditis et al. 2001).

\section{Mechanism of factor binding to the ribosome}

The association of both EF-Tu:GTP:aminoacyl-tRNA and EF-G with the ribosome takes place more rapidly than expected for a random encounter of two particles of this size (Rodnina et al. 1996; Savelsbergh et al. 2003). Our data identify the L12 CTDs as interaction sites for the factors. Thus, the unexpectedly high rate of factors binding to the ribosome may be explained by an increase of the encounter frequency of the ternary complex or EF-G due to multiple copies of L12, leading to a higher association rate by introducing a favorable statistical factor (Rodnina et al. 1996). This suggestion is supported by the structural model of the stalk (Figure 26): the L12 CTDs can reach far out into solution, where they can 'catch' translation factors and 'hand them over' to the ribosomal factor binding site, thus efficiently restricting factor diffusion and leading to rapid recruitment. The long, unstructured L12 hinge regions and the flexible connection of the L10 helix $\alpha 8$-L12 NTD portion to the stalk base could allow the interaction of the translation factors with their ribosome binding site after initial capture by the L12 CTDs. 


\section{Mechanism of GTPase stimulation}

The L12 CTDs are responsible for an about 1000-fold stimulation of GTP hydrolysis by EF-Tu and EF-G. GTPase activation can be achieved either by promoting conformational rearrangements of the factors that correctly position their own catalytic groups in the active site or by donating additional catalytic groups in trans. The unique, highly conserved arginine residue in the CTD of L12 is not essential for the activation, excluding an 'arginine finger'-type mechanism (present data and (Savelsbergh et al. 2000)). Similarly, none of the other conserved, surface exposed amino acid residues in the CTD alone is responsible for the activation. These findings suggest that L12 facilitates GTP hydrolysis by stabilizing the GTPase transition state of the factors, rather than by providing residues involved in catalysis. This mechanism of activation resembles that of the regulators of G-protein signaling (RGS), which stimulate GTP hydrolysis in G $\alpha$ proteins (for review see (Vetter and Wittinghofer 1999)).

Cryo-EM reconstructions showed extensive interactions of the $\mathrm{G}$ domains of both EF-Tu and EF-G with the SRL of 23S rRNA (Agrawal et al. 1998; Stark et al. 2002; Valle et al. 2003b), indicating that the SRL may stabilize the transition state conformation of the factors. Single-molecule fluorescence measurements indicated that cleavage of the SRL blocks EF-Tu in a state before GTP hydrolysis (Blanchard et al. 2004). Other contacts, which may contribute to GTP hydrolysis, include ribosomal protein L11 and the L11binding region of 23S rRNA (Agrawal et al. 2001). L12 represents a third ribosomal element important for stimulation of GTP hydrolysis. Via its CTD it may both facilitate positioning the factors relative to other ribosomal components contributing to catalysis and stabilize the active conformation of the factors. Therefore, it can be envisaged that the L12 CTDs use their high freedom of motion to reach back towards the ribosome-bound factors to stimulate their GTPase activity. The requirement for additional signals for full stimulation of the GTPase activity, such as the interaction with SRL or L11, would help to avoid premature GTP hydrolysis during initial factor binding.

\section{E. Cross-kingdom similarities and differences in the stalk}

The archaeal L10E NTD is structurally homologous to bacterial L10 NTD, consistent with a similar mode of binding to the 23S rRNA. Sequence conservation between archaeal and eukaryotic L10 orthologs (Figure 22) suggests that the eukaryotic P0 
proteins are also similarly structured in the N-terminal part, and thus presumably bind to the $28 \mathrm{~S}$ rRNA in a similar way. The notion of a structurally conserved rRNA binding module in L10 orthologs across the kingdoms, which seems to recognize a conserved fold in the rRNAs, explains why archaeal or eukaryotic orthologs of the L10:L12 complex can be attached to bacterial ribosomes and vice versa (Sanchez-Madrid et al. 1981; StofflerMeilicke and Stoffler 1991; Uchiumi et al. 1999; Uchiumi et al. 2002; Terasaki et al. 2004; Nomura et al. 2006). Eukaryotic P0:P1/P2 complexes transplanted onto E. coli ribosomes are functional, provided that eukaryotic translation factors are supplied.

However, beyond the rRNA binding domain, eukaryotic L10 orthologs differ from their bacterial counterparts in structure and in the mode of interaction with L12 orthologs (for a recent review see (Gonzalo and Reboud 2003)). E.g. eukaryotic P0 proteins are considerably longer than bacterial L10 (by some 150 residues) and the ultimate C-terminal part of L10 may encompass a P1/P2-like structure (Gonzalo and Reboud 2003). Eukaryotes maintain two (P1 and P2, some with subfamilies), plants possibly three (an additional P3) families of acidic phosphoproteins, which interact with P0. The more intricate organization of the $\mathrm{P} 0: \mathrm{P} 1 / \mathrm{P} 2(\mathrm{P} 3)$ complexes in higher organisms compared to the bacterial L10:L12 complexes may reflect additional functions in translation regulation (Gonzalo and Reboud 2003). The present results indicate that free bacterial L12 exchanges only very slowly with ribosome-bound L12. This situation seems to be decisively different in eukaryotes where exchange of $\mathrm{P} 1 / \mathrm{P} 2$ and phosphorylation during translation were suggested to modify expression of some mRNAs (Gonzalo and Reboud 2003). 


\section{$\underline{\text { Outlook }}$}

\section{I. $\mathrm{L10}_{\Delta \mathrm{DBS}}: \mathrm{L} 12$ complexes from Thermotoga maritima}

Structures at 2.3, 2.1 and $1.9 \AA$ resolution were determined for a bacterial L10:(L12 NTD) 6 complex. As previously described, their analysis demonstrated that the C-terminal domain of L10 carrying L12 NTDs is flexibly connected to its NTD via a short "hinge". Despite the certain degree of mobility of this complex, three well-diffracting crystal forms were easily obtained. Conversely, attempts to crystallize full-length L10:(L12)6 complexes resulted in another truncated variant corresponding to L10:(L12 NTD)6. The trimming occured in the hinge regions, which are most probably in an extended, random coil conformation and thereby susceptible to proteolysis. These results suggested that although the inherent flexibility of this complex is difficult to surmount, shorter complex variants could cope with some flexibility and result in well-resolved structures. In this view, terminal deletion variants of L10 were designed to carry either one or two full-length L12 dimers $\left(\mathrm{L} 10_{\triangle \mathrm{DBS}}: \mathrm{L} 12\right.$; DBS, dimer binding site). This approach was possible as the Cterminal $\alpha$-helix of L10 is kinked in two positions, resulting in three almost equal segments, each of which accommodating one L12 dimer. The resulting L10 ${ }_{\triangle \mathrm{DBS}}$ :L12 complexes would reveal the structure of the full-length L12 molecules in the context of the L10:L12 complex. In addition, the placement of these truncated complexes on the ribosome would address the issue of whether L12 dimers attached to different sites in the L10 molecule perform different functions.

\section{A. Production of the $t m a L 10_{\Delta 2 \mathrm{DBs}}: \mathrm{L} 12$ complex}

In order to generate a L10:L12 complex carrying one $\left(\operatorname{tmaL} 10_{\Delta 2 \mathrm{DBS}}: \mathrm{L} 12\right)$ or two (tmaL10 $\left.0_{\Delta 1 \mathrm{DBS}}: \mathrm{L} 12\right)$ dimers of L12, stop codons were introduced after codon 153 and 163 respectively, in the plasmid used for tmaL10 production (Table 8). tmaL10 $0_{\Delta 2 \mathrm{DBS}}$ and tmaL12 $2_{\Delta 1 \mathrm{DBS}}$ encoding plasmids were then co-transformed with the plasmid used for tmaL12 production (Wahl et al. 2000b) and subsequently co-expressed in Rosetta(DE3) strain (Figure 27-1). So far, only the production of $t m a \mathrm{~L} 10_{\Delta 2 \mathrm{DBS}}: \mathrm{L} 12$ was carried out (Figure 27-2,3). The purification procedure was performed as described for tmaL10:(L12 
NTD) ${ }_{6}$ (see section III.A.1., Results). Fractions containing the complex were combined, concentrated to $10 \mathrm{mg} / \mathrm{ml}$ and subjected to crystallization.

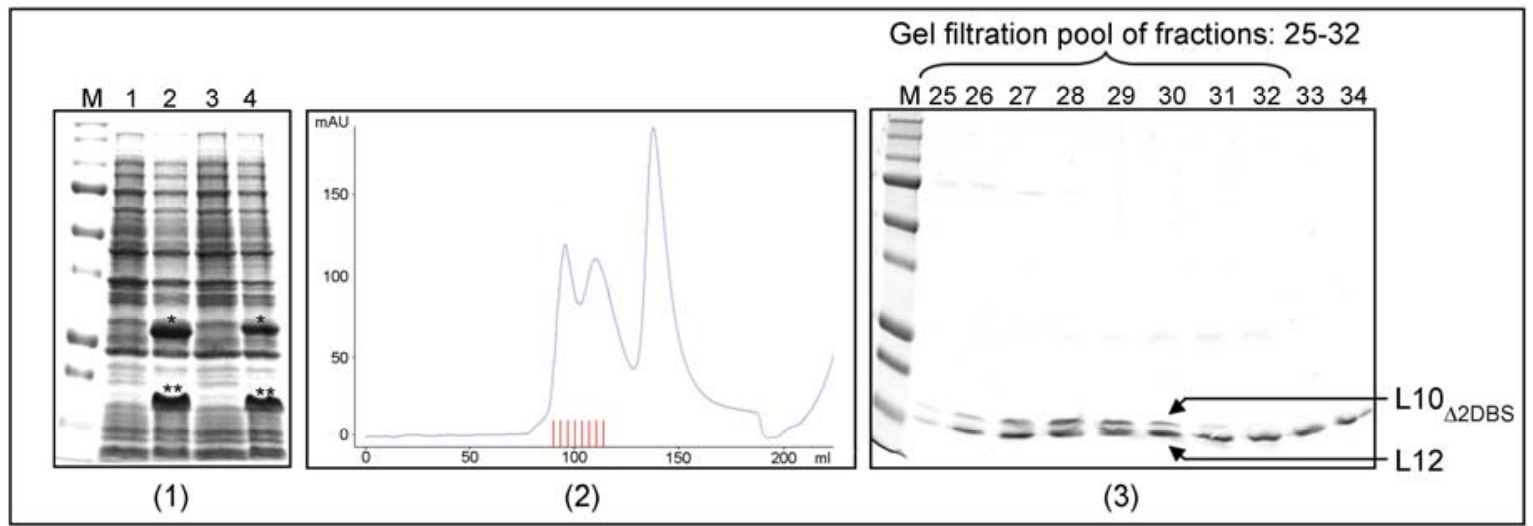

Figure 27. (1) Expression of $t m a \mathrm{~L} 10_{\triangle \mathrm{DBS}}: \mathrm{L} 12$ complexes. Prior induction (lane 1) and post induction (lane 2) phases of tmaL10 ${ }_{\triangle 2 \mathrm{DBS}}:$ L12 complex expression; prior induction (lane 3) and post induction (lane 4) phases of $t m a \mathrm{~L} 10_{\Delta \mathrm{DBBS}}: \mathrm{L} 12$ complex expression; $(*)$ overexpressed tmaL10 $10_{\Delta 2 \mathrm{DBS}}$ and $t m a \mathrm{~L} 10_{\Delta 1 \mathrm{DBS}}$; $(* *)$ overexpressed full-length L12. (2) Chromatogram representing the elution profile of the tmaL10 $0_{\Delta 2 \mathrm{DBS}}: \mathrm{L} 12$ complex on the Superdex 75 gel filtration column, as the last step of purification. (3) Fractions containing the purified complex (25-32) were combined and concentrated to $10 \mathrm{mg} / \mathrm{ml}$.

\section{B. Crystallization trials of the $t m a L_{10}{ }_{\Delta 2 D B S}: \mathrm{L} 12$ complex}

A high-throughput crystallization experiment was initiated on the in-house vapor diffusion nano drop robot. 288 different conditions were screened for the $t m a \mathrm{~L} 10_{\Delta 2 \mathrm{DBS}}: \mathrm{L} 12$ complex using sparse matrix from Nextal Biotechnologies and Hampton Research. The outcome of these experiments is currently under investigation.

\section{II. tmaL12 CTD and its interaction with elongation factors}

Several lines of evidence indicated the involvement of L12 in various translation factorrelated functions. The placement of the isolated tmaL10:(L12 NTD) 6 structure on the large ribosomal subunit and the resulting model of the L7/L12 stalk, suggested that L12 CTDs represent the active sites of the stalk (Figure 26). Indeed, carefully designed fast kinetic experiments pinpointed L12 CTDs as primary interaction sites for factor GTPases and as active stimulators of their GTP hydrolysis (U. Kothe, M.V. Rodnina, see section II. C. and D., Discussion). As a mechanism of GTP hydrolysis, it was hypothesized that the L12 CTDs could either remain bound to the factors' $G$ domain during movement of the factors to the ribosomal factor binding site or they could reach back towards the ribosome-bound 
factor to stimulate their GTPase activities. Consequently, attempts to identify interactions between L12 CTD and the G domains of elongation factors (EF-Tu and EF-G) by means of X-ray crystallography and Biacore, using purified proteins from T. maritima, were made.

\section{A. Production of the tmaL12 CTD and of tmaEF-Tu(Gd)}

Both L12 CTD and the G-domain of EF-Tu (EF-Tu(Gd)) were expressed from pETM-11 (Figure 28A-1) and pET22b(+) vectors in Rosetta(DE3) strain. The pETM-11 vector provide an $\mathrm{N}$-terminal $\mathrm{His}_{6}$-tag, whereas the pET22b(+) has no affinity tag (Table 5). In order to detect a potential interaction between L12 CTD and EF-Tu(Gd), a $\mathrm{His}_{6}$-tag L12 CTD fusion protein from pETM-11 was co-transformed and subsequently coexpressed with $\mathrm{EF}-\mathrm{Tu}(\mathrm{Gd})$ from $\mathrm{pET} 22 \mathrm{~b}(+)$ and vice versa. Only minute amounts of tmaEF-Tu(Gd) protein were co-purified with His ${ }_{6}$-tmaL12 CTD on Ni-NTA affinity matrix (and vice versa), suggesting a very weak interaction between them (data not shown). Thus, for subsequent crystallographic and interaction studies, both proteins were produced individually from pETM-11 vector and combined in the end (Figure 28A-2, 3, 4, 5). The purification procedure used was as described previously for the tmaL10:(L12 NTD) 6 (see section III.A.1., Results). 

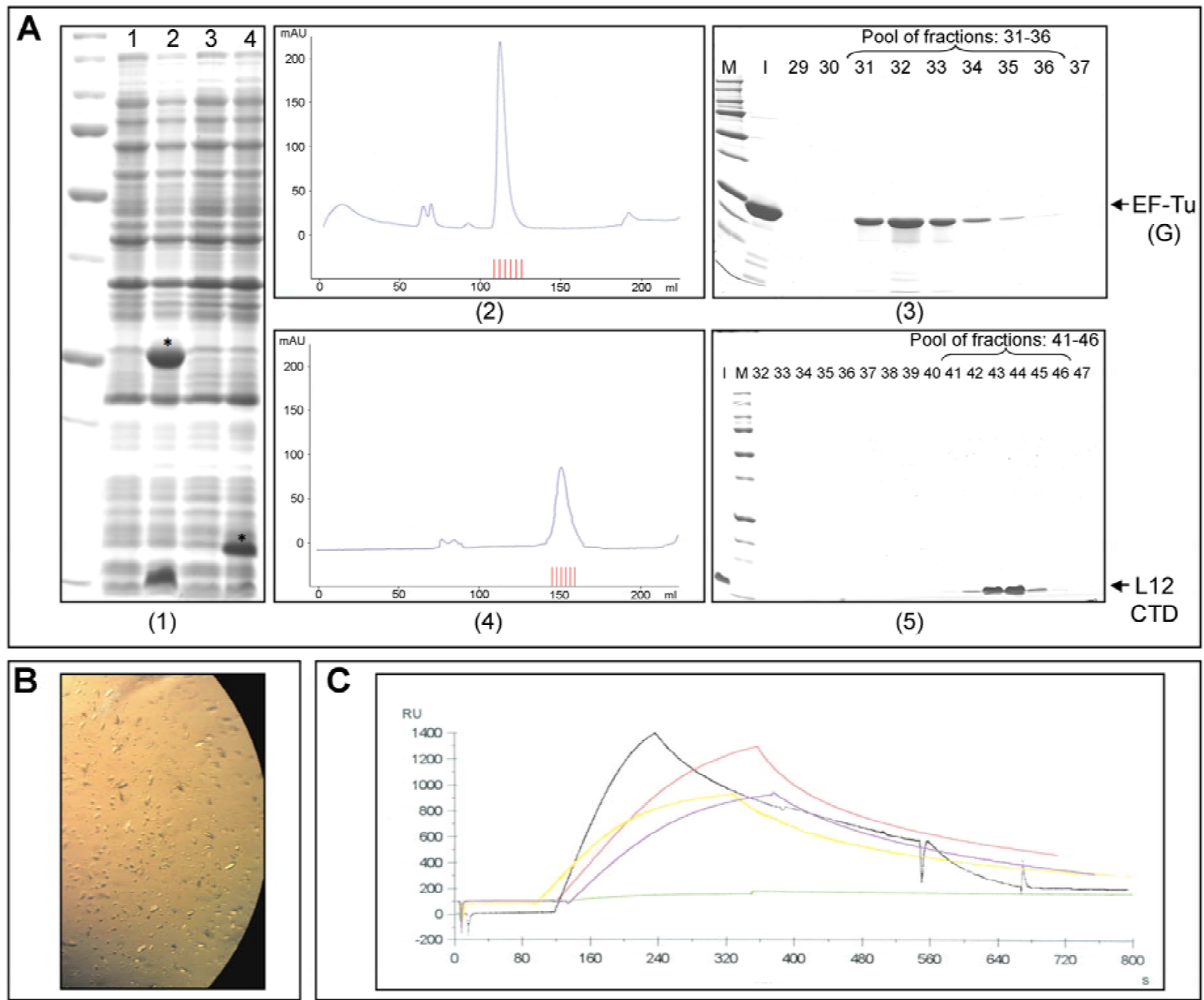

Figure 28. (A) (1) prior induction (lane 1) and post induction (lane 2) phases of $\mathrm{His}_{6}-t m a \mathrm{EF}-\mathrm{Tu}(\mathrm{Gd})$ expression; prior induction (lane 3) and post induction (lane 4) phases of His $_{6}$-tmaL12 CTD expression; (*) overexpressed proteins tmaEF-Tu(Gd) and tmaL12 CTD, respectively. (2) Chromatogram representing the elution profile of the tmaEF-Tu(Gd) on a Superdex 75 gel filtration column, as a last step of the purification procedure. (3) Fractions (31-36) containing the purified protein were combined and concentrated to 10 $\mathrm{mg} / \mathrm{ml}$. (4) Chromatogram representing the elution profile of the tmaL12 CTD on a Superdex 75 gel filtration column, as a last step of the purification procedure. (5) Fractions (41-46) containing the purified protein were combined and concentrated to $8 \mathrm{mg} / \mathrm{ml}$. I, input sample, collected before the gel filtration chromatographic step. (B) tmaL12 CTD:EF-Tu(Gd):GMPPNP putative crystals. (C) Sensorgram representing changes in the resonance signal [RU] over time [s] for the tmaL12 CTD (yellow curve), tmaL12 CTD:EF-Tu(Gd) (violet curve), tmaL12 CTD:EF-Tu(Gd):GTP (black curve), tmaEF-G (green curve), tmaL12 CTF:EF-G (red curve). The sensorgram is a direct representation of the interaction between molecules in real time. $1000 \mathrm{RU}$ (resonance units) represent a shift in the resonance angle of $0.1^{\circ}$. The Biacore measurements were performed together with Dr. Igor Agapov, Witten. 


\section{B. Crystallization trials}

672 different conditions (Nextal Biotechnologies) were set up at $20^{\circ} \mathrm{C}$ for each of the following complexes:

(1) tmaL12 CTD:EF-Tu(Gd) :GDP in a molar ratio of $1: 1: 15$

(2) tmaL12 CTD:EF-Tu(Gd) :GMPPNP in a molar ratio of 1:1:20 (where GMPPNP is a non-hydrolysable GTP analogue)

(3) tmaL12 CTD:EF-G :GMPPNP in a molar ratio of 1:1:20 ( full-length tmaEF-G was kindly provided by A. Savelsbergh, Witten)

One condition belonging only to the L12 CTD:EF-Tu(Gd):GMPPNP yielded crystals represented in Figure 28B. Further screening and testing of these crystals are ongoing.

\section{Interaction studies of the tmaL12 CTD and elongation factors by Biacore}

In parallel with crystallization trials, the association ability of tmaL12 CTD with either elongation factor was monitored using surface plasmon resonance (Biacore). A His $6^{-}$ tagged tmaL12 CTD fusion protein was produced for this purpose. After controlling that only this protein can bind onto the surface of the Ni-NTA sensor chip, samples containing tmaL12 CTD together with tmaEF-Tu(Gd) or tmaEF-G were injected over this surface at a constant flow rate through a microfluidic channel system. His ${ }_{6}$-tmaL12 CTD alone bound to the Ni-NTA chip and produced a SPR signal of approximately 900 resonance units (RU) (Figure 28C, yellow curve). No variation in the SPR could be detected in the presence of tmaEF-Tu(Gd) as compared to the sole tmaL12 CTD (Figure 28C, violet curve). The latter result indicated that the binding between the two components is very weak or absent. The time-dependent dissociation was also found similar to the control sample. In the next approach, the addition of a complex of tmaEF-Tu(Gd):GTP (with GTP incorporated in a pyruvate kinase-dependent reaction) had also no effect on the time-dependent dissociation of the His ${ }_{6}$-tmaL12 CTD (Figure 28C, black curve). The observed increase in the amplitude of the sensorgram is only related to almost doubly-concentrated ligand (His 6 tmaL12 CTD) and analyte (tmaEF-Tu(Gd):GTP) used in this experiment. To rule out any unspecific binding of tmaEF-G to the Ni-NTA surface or to His 6 -tagged tmaL12 CTD, 
tmaEF-G was directly added onto the Ni-NTA chip (Figure 28C, green curve). No binding of tmaEF-G onto the Ni-NTA chip was detected. Interestingly, when a premix of $\mathrm{His}_{6}$ tmaL12 CTD with tmaEF-G was added onto the Ni-NTA chip, the SPR signal was found increased by a factor of 1.5 as compared to the sole $\mathrm{His}_{6}$-tmaL12 CTD, indicating a direct interaction between the two components (Figure 28C, red curve). Additionally, the dissociation of this complex was slower than for the control protein. Similar SPR data were reported for the eukaryotic counterparts, where eEF-2 was found to interact specifically in vitro with $\mathrm{P}$ proteins, however, with a higher affinity for P1 than for P2 (Bargis-Surgey et al. 1999).

Taken together, these results suggest that in isolation, EF-Tu(Gd) and L12 CTD molecules do not seem to associate or their association is very weak. The latter possibility is supported by the fact that small amounts of tmaEF-Tu(Gd) were co-purified together with His $_{6}$-tmaL12 CTD on Ni-NTA beads (data not shown). In contrast, EF-G and L12 CTD appear to associate in isolation. However, the relatively fast dissociation in time does not suggest a tight interaction between the two components. These results are in line with kinetic data regarding the effect of protein L12 on the GTP hydrolysis by EF-Tu and EF-G in isolation. L12 strongly stimulated GTP hydrolysis by EF-G (Savelsbergh et al. 2000), but not by EF-Tu, indicating that for the latter event additional ribosomal components are required (Piepenburg et al. 2000).

\section{L11:L12 CTD complexes from Thermotoga maritima}

Highly mobile L12 CTDs promote the recruitment of translation factors to the ribosome and stimulate GTP hydrolysis by the ribosome-bound factors through stabilization of their active GTPase conformation. However, in the latter process, the involvement of additional elements, including protein L11 or sarcin-ricin loop seem to be required to prevent premature GTP hydrolysis during initial factor binding by L12 CTD. In order to identify a potential interaction between protein L11 and L12 CTD, a crystallographic experiment was initiated and a preliminary binding study by isothermal titration calorimetry was performed. 


\section{A. Production of tmaL11}

L11 was expressed from pETM-11 and was found to preferentially require E. coli BL21(DE3) strain for propagation (Figure 29A-1). Following its expression, the His 6 -L11 fusion protein was purified via Ni-NTA affinity chromatography. After removal of the His $_{6}$-tag, the protein was further purified by heat treatment and cation exchange chromatography. The final preparation was concentrated to $10 \mathrm{mg} / \mathrm{ml}$ (Figure 29A-2,3).
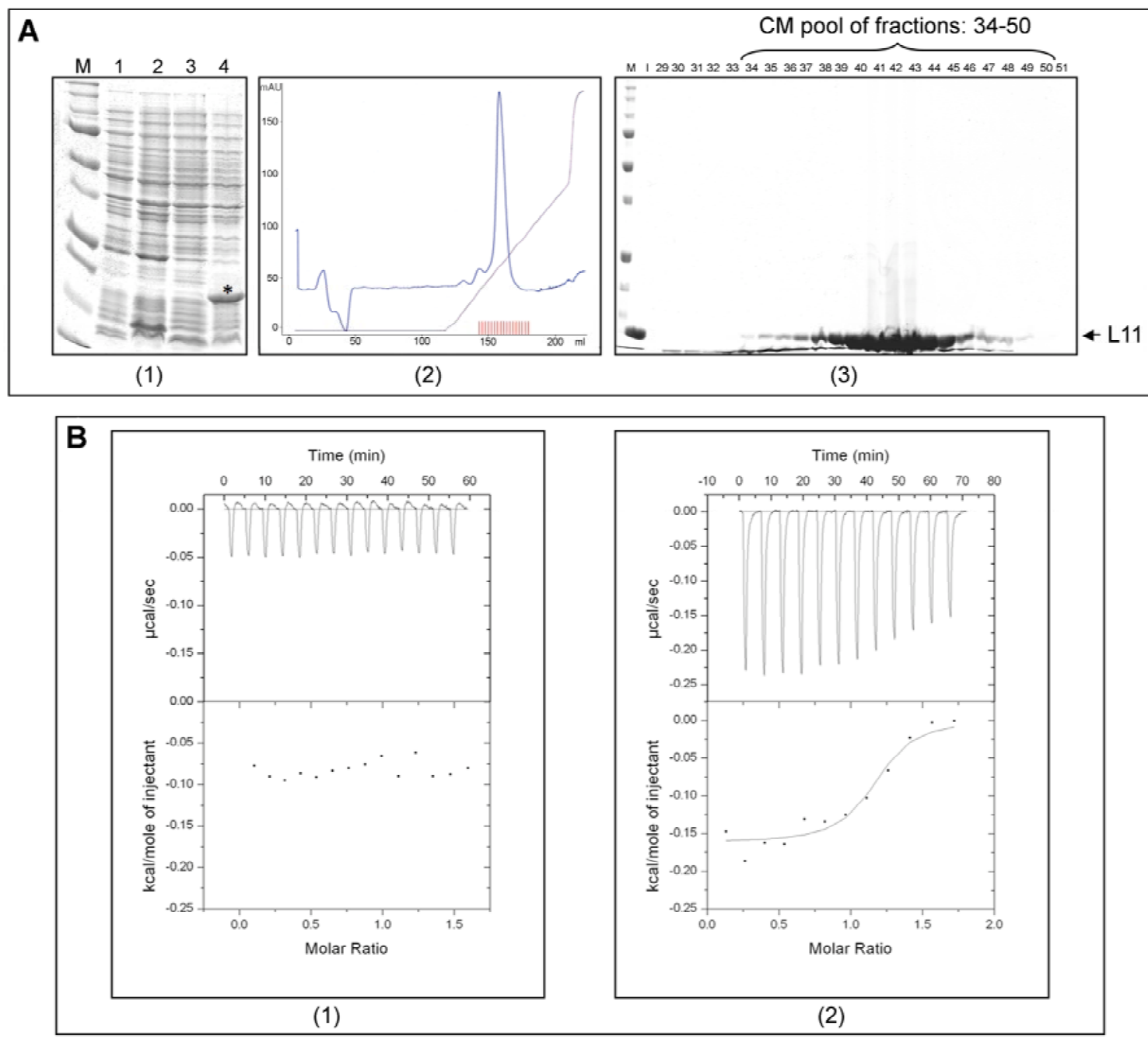

Figure 29. (A) Expression and purification of tmaL11. (1) prior induction (lane 1) and post induction (lane 2) phases of tmaL11 expression in Rosetta(DE3) strain, no expression was detected; prior induction (lane 3) and post induction (lane 4) phases of tmaL11 expression in BL21(DE3) strain, (*) overexpressed protein. (2) Chromatogram representing the elution profile of the tmaL11 on a CM cation exchange column, as the last step of the purification procedure. (3) Fractions (34-50) containing the purified protein were combined and concentrated to $10 \mathrm{mg} / \mathrm{ml}$. (B) (1) ITC of the system's buffer as control, $\Delta \mathrm{H}=-0.05 \mathrm{kcal} / \mathrm{mol}$. (2) ITC data for titration of $135 \mu \mathrm{M}$ tmaL12 CTD with 12 injections of $1 \mathrm{mM}$ tmaL11. Upper panel: raw power $(\mu \mathrm{cal} / \mathrm{sec})$ versus time tracing. At each injection an exothermic spike is seen. The area under each spike is proportional to the heat of binding of tmaL11 to tmaL12 CTD; lower panel: amount of heat measured at each injection normalized to the number of moles of tmaL11 injected $(\mathrm{kcal} / \mathrm{mol})$ versus molar ratio of cumulative tmaL11 added to the tmaL12 CTD in the cell. Data analysis indicated that the saturation will be reached at 2:1 molar ratio of tmaL12 CTD:tmaL11. A binding of $\Delta \mathrm{H}=-0.16 \mathrm{kcal} / \mathrm{mol} t m a \mathrm{~L} 11$, and an equilibrium dissociation constant $\mathrm{k}_{\mathrm{d}}=1.9 \mu \mathrm{M}$ were obtained. 


\section{B. Crystallization trials of tmaL11:L12 CTD}

tmaL11 and tmaL12 CTD proteins were mixed in a molar ratio of $1: 1$, at a final concentration of $9 \mathrm{mg} / \mathrm{ml}$, incubated approximately $15 \mathrm{~h}$ at $4{ }^{\circ} \mathrm{C}$ to allow complex formation and subjected to crystallization trials. 1152 different conditions (Nextal Biotechnologies) were next screened for the $\operatorname{tmaL11:L12~CTD}$, at $20^{\circ} \mathrm{C}$, on the in-house vapor diffusion dispensing apparatus. These experiments are ongoing.

\section{Interaction study of the tmaL11 and tmaL12 CTD by isothermal titration calorimetry}

A putative interaction between tmaL12 CTD and tmaL11 was assessed by microcalorimetry. Prior to this determination, a control sample representing the buffer of the sistem was analysed (Figure 29B-1). Subsequently, a titration of $135 \mu \mathrm{M}$ tmaL12 CTD (placed in a calorimetric cell) with 12 injections of $1 \mathrm{mM}$ tmaL11 was performed (Figure 29B-2). Increasing amounts of tmaL11 were added to tmaL12 CTD at the indicated molar ratios in a microcalorimeter, and the energy required to compensate for the binding enthalpy in order to reestablish thermal equilibrium after each addition, was measured and plotted in microcalories per second (Figure 29B-2, upper panel). From the plot of the binding enthalpies (Figure 29B-2, lower panel), transitions due to saturation appeared to be achieved for a stoichiometry of 1 mole of tmaL11 to approximately 2 moles of tmaL12 CTD. The dissociation equilibrium constant $\left(\mathrm{k}_{\mathrm{d}}\right)$ was $1.9 \mu \mathrm{M}$, suggesting a weak interaction between the two components in isolation. Further testing of this interaction is currently under investigation. The accuracy of the above mentioned experiment needs to be ascertained by measurements with an increased amount of both cell and syringe reactants.

Despite the low affinity between these two components in isolation, several lines of evidence suggested an interaction between tmaL12 CTD and tmaL11 on the ribosome. Cryo-EM studies revealed that the G domains of factor GTPases (in their GDP bound conformation) form an arc-like connection with the base of the L7/L12 stalk (Stark et al. 1997a; Agrawal et al. 1998). Subsequently, the element at the base of the stalk implicated in the formation of this bridge was identified as L11 NTD (Agrawal et al. 2001). Recently, another cryo-EM analysis further delineated boundaries of the G' domain of EF-G and L11 
NTD and tentatively positioned between them one copy of L12 CTD (Datta et al. 2005). This location of L12 CTD is in agreement with cross-linking (Dey et al. 1998) and EM (Montesano-Roditis et al. 2001) data that placed the L12 CTD in the vicinity of L11. In the present calorimetric study L11 and L12 CTD exhibit a low affinity for each other in isolation, suggesting that additional ribosomal elements or factor contacts are required for their proper association and function. Remarkably, in the above mentioned study of Datta and coworkers, the L12 CTD was seen near the L11 NTD lobe, both in the presence and absence of EF-G. During the course of EF-G-dependent reactions, L12 CTD underwent considerable conformational changes in association with L11 NTD. Furthermore, the L12 CTD was found slightly twisted toward the G' domain of EF-G upon GTP hydrolysis. The positioning of the L12 CTD was not close to the GTP catalysis center of EF-G, in agreement with our observation that L12 CTD activates GTP hydrolysis allosterically rather than catalytically. In addition to stimulating GTP hydrolysis by EF-G, the L12 CTD could also assist the release of the factor from the ribosome. Following GTP hydrolysis, the domain V of EF-G pushes outward the L11 NTD. This movement is transmitted to neighboring L12 CTD, which in turn would signal the dissociation of EF-G:GDP from the ribosome. Thus, L12 CTD is portrayed as a "multi-task" element, with dynamic and diverse role in the ribosome function, including the recognition and recruitment of various translational factors, the triggering of GTP hydrolysis-related events, and finally the removal of those factors from the ribosome. 


\section{$\underline{\text { References }}$}

Ævarsson, A., Brazhnikov, E., Garber, M., Zheltonosova, J., Chirgadze, Y., al-Karadaghi, S., Svensson, L.A., and Liljas, A. 1994. Three-dimensional structure of the ribosomal translocase: elongation factor $G$ from Thermus thermophilus. Embo $J$ 13(16): 36693677.

Agrawal, R.K., Heagle, A.B., Penczek, P., Grassucci, R.A., and Frank, J. 1999. EF-Gdependent GTP hydrolysis induces translocation accompanied by large conformational changes in the 70S ribosome. Nat Struct Biol 6(7): 643-647.

Agrawal, R.K., Linde, J., Sengupta, J., Nierhaus, K.H., and Frank, J. 2001. Localization of L1 1 protein on the ribosome and elucidation of its involvement in EF-G-dependent translocation. J Mol Biol 311(4): 777-787.

Agrawal, R.K., Penczek, P., Grassucci, R.A., and Frank, J. 1998. Visualization of elongation factor $\mathrm{G}$ on the Escherichia coli $70 \mathrm{~S}$ ribosome: the mechanism of translocation. Proc Natl Acad Sci U S A 95(11): 6134-6138.

Al-Karadaghi, S., Kristensen, O., and Liljas, A. 2000. A decade of progress in understanding the structural basis of protein synthesis. Prog Biophys Mol Biol 73(2-4): 167-193.

Allen, G.S., Zavialov, A., Gursky, R., Ehrenberg, M., and Frank, J. 2005. The cryo-EM structure of a translation initiation complex from Escherichia coli. Cell 121(5): 703712 .

Ban, N., Nissen, P., Hansen, J., Capel, M., Moore, P.B., and Steitz, T.A. 1999. Placement of protein and RNA structures into a 5 A-resolution map of the $50 \mathrm{~S}$ ribosomal subunit. Nature 400(6747): 841-847.

Ban, N., Nissen, P., Hansen, J., Moore, P.B., and Steitz, T.A. 2000. The complete atomic structure of the large ribosomal subunit at 2.4 A resolution. Science 289(5481): 905920.

Bargis-Surgey, P., Lavergne, J.P., Gonzalo, P., Vard, C., Filhol-Cochet, O., and Reboud, J.P. 1999. Interaction of elongation factor eEF-2 with ribosomal P proteins. Eur J Biochem 262(2): 606-611.

Barton, G.J. 1993. ALSCRIPT: a tool to format multiple sequence alignments. Protein Eng 6(1): 37-40.

Beauclerk, A.A., Cundliffe, E., and Dijk, J. 1984. The binding site for ribosomal protein complex L8 within 23 s ribosomal RNA of Escherichia coli. J Biol Chem 259(10): 6559-6563.

Berchtold, H., Reshetnikova, L., Reiser, C.O., Schirmer, N.K., Sprinzl, M., and Hilgenfeld, R. 1993. Crystal structure of active elongation factor $\mathrm{Tu}$ reveals major domain rearrangements. Nature 365(6442): 126-132.

Blanchard, S.C., Gonzalez, R.L., Kim, H.D., Chu, S., and Puglisi, J.D. 2004. tRNA selection and kinetic proofreading in translation. Nat Struct Mol Biol 11(10): 1008-1014.

Bocharov, E.V., Gudkov, A.T., and Arseniev, A.S. 1996. Topology of the secondary structure elements of ribosomal protein L7/L12 from E. coli in solution. FEBS Lett 379(3): 291294.

Bocharov, E.V., Sobol, A.G., Pavlov, K.V., Korzhnev, D.M., Jaravine, V.A., Gudkov, A.T., and Arseniev, A.S. 2004. From structure and dynamics of protein L7/L12 to molecular switching in ribosome. J Biol Chem 279(17): 17697-17706.

Boelens, R. and Gualerzi, C.O. 2002. Structure and function of bacterial initiation factors. Curr Protein Pept Sci 3(1): 107-119.

Boublik, M., Hellmann, W., and Roth, H.E. 1976. Localization of ribosomal proteins L7L12 in the $50 \mathrm{~S}$ subunit of Escherichia coli Ribosomes by electron microscopy. $J$ Mol Biol 107(4): 479-490. 
Bourne, H.R., Sanders, D.A., and McCormick, F. 1991. The GTPase superfamily: conserved structure and molecular mechanism. Nature 349(6305): 117-127.

Bradford, M.M. 1976. A rapid and sensitive method for the quantitation of microgram quantities of protein utilizing the principle of protein-dye binding. Anal Biochem 72: 248-254.

Brodersen, D.E., Clemons, W.M., Jr., Carter, A.P., Morgan-Warren, R.J., Wimberly, B.T., and Ramakrishnan, V. 2000. The structural basis for the action of the antibiotics tetracycline, pactamycin, and hygromycin $\mathrm{B}$ on the $30 \mathrm{~S}$ ribosomal subunit. Cell 103(7): 1143-1154.

Brodersen, D.E., Clemons, W.M., Jr., Carter, A.P., Wimberly, B.T., and Ramakrishnan, V. 2002. Crystal structure of the $30 \mathrm{~S}$ ribosomal subunit from Thermus thermophilus: structure of the proteins and their interactions with 16 S RNA. J Mol Biol 316(3): 725768.

Brosius, J., Dull, T.J., and Noller, H.F. 1980. Complete nucleotide sequence of a $23 \mathrm{~S}$ ribosomal RNA gene from Escherichia coli. Proc Natl Acad Sci U S A 77(1): 201-204.

Brot, N., Tate, W.P., Caskey, C.T., and Weissbach, H. 1974. The requirement for ribosomal proteins L7 and L12 in peptide-chain termination. Proc Natl Acad Sci U S A 71(1): 8992.

Brot, N. and Weissbach, H. 1981. Chemistry and biology of E. coli ribosomal protein L12. Mol Cell Biochem 36(1): 47-63.

Brunger, A.T., Adams, P.D., Clore, G.M., DeLano, W.L., Gros, P., Grosse-Kunstleve, R.W., Jiang, J.S., Kuszewski, J., Nilges, M., Pannu, N.S., Read, R.J., Rice, L.M., Simonson, T., and Warren, G.L. 1998. Crystallography \& NMR system: A new software suite for macromolecular structure determination. Acta Crystallogr D Biol Crystallogr 54(Pt 5): 905-921.

Budisa, N., Steipe, B., Demange, P., Eckerskorn, C., Kellermann, J., and Huber, R. 1995. High-level biosynthetic substitution of methionine in proteins by its analogs 2aminohexanoic acid, selenomethionine, telluromethionine and ethionine in Escherichia coli. Eur J Biochem 230(2): 788-796.

Bushuev, V.N., Gudkov, A.T., Liljas, A., and Sepetov, N.F. 1989. The flexible region of protein $\mathrm{L} 12$ from bacterial ribosomes studied by proton nuclear magnetic resonance. $J$ Biol Chem 264(8): 4498-4505.

Cameron, D.M., Thompson, J., March, P.E., and Dahlberg, A.E. 2002. Initiation factor IF2, thiostrepton and micrococcin prevent the binding of elongation factor $G$ to the Escherichia coli ribosome. J Mol Biol 319(1): 27-35.

Carter, A.P., Clemons, W.M., Brodersen, D.E., Morgan-Warren, R.J., Wimberly, B.T., and Ramakrishnan, V. 2000. Functional insights from the structure of the $30 \mathrm{~S}$ ribosomal subunit and its interactions with antibiotics. Nature 407(6802): 340-348.

Carter, A.P., Clemons, W.M., Jr., Brodersen, D.E., Morgan-Warren, R.J., Hartsch, T., Wimberly, B.T., and Ramakrishnan, V. 2001. Crystal structure of an initiation factor bound to the 30S ribosomal subunit. Science 291(5503): 498-501.

Casiano, C., Matheson, A.T., and Traut, R.R. 1990. Occurrence in the archaebacterium Sulfolobus solfataricus of a ribosomal protein complex corresponding to Escherichia coli (L7/L12)4.L10 and eukaryotic (P1)2/(P2)2.P0. J Biol Chem 265(31): 1875718761.

Cate, J.H., Yusupov, M.M., Yusupova, G.Z., Earnest, T.N., and Noller, H.F. 1999. X-ray crystal structures of $70 \mathrm{~S}$ ribosome functional complexes. Science 285(5436): 20952104.

Chandra Sanyal, S. and Liljas, A. 2000. The end of the beginning: structural studies of ribosomal proteins. Curr Opin Struct Biol 10(6): 633-636. 
Chinali, G. and Parmeggiani, A. 1982. Differential modulation of the elongation-factor-G GTPase activity by tRNA bound to the ribosomal A-site or P-site. Eur J Biochem 125(2): 415-421.

Clemons, W.M., Jr., May, J.L., Wimberly, B.T., McCutcheon, J.P., Capel, M.S., and Ramakrishnan, V. 1999. Structure of a bacterial 30S ribosomal subunit at $5.5 \mathrm{~A}$ resolution. Nature 400(6747): 833-840.

Correll, C.C., Munishkin, A., Chan, Y.L., Ren, Z., Wool, I.G., and Steitz, T.A. 1998. Crystal structure of the ribosomal RNA domain essential for binding elongation factors. Proc Natl Acad Sci U S A 95(23): 13436-13441.

Cundliffe, E. and Thompson, J. 1981. Concerning the mode of action of micrococcin upon bacterial protein synthesis. Eur J Biochem 118(1): 47-52.

Czworkowski, J., Wang, J., Steitz, T.A., and Moore, P.B. 1994. The crystal structure of elongation factor G complexed with GDP, at 2.7 A resolution. Embo $J$ 13(16): 36613668.

Datta, P.P., Sharma, M.R., Qi, L., Frank, J., and Agrawal, R.K. 2005. Interaction of the G' domain of elongation factor $\mathrm{G}$ and the $\mathrm{C}$-terminal domain of ribosomal protein $\mathrm{L} 7 / \mathrm{L} 12$ during translocation as revealed by cryo-EM. Mol Cell 20(5): 723-731.

De Vendittis, E., Masullo, M., and Bocchini, V. 1986. The elongation factor G carries a catalytic site for GTP hydrolysis, which is revealed by using 2-propanol in the absence of ribosomes. J Biol Chem 261(10): 4445-4450.

Dell, V.A., Miller, D.L., and Johnson, A.E. 1990. Effects of nucleotide- and aurodox-induced changes in elongation factor $\mathrm{Tu}$ conformation upon its interactions with aminoacyl transfer RNA. A fluorescence study. Biochemistry 29(7): 1757-1763.

Deusser, E. 1972. Heterogeneity of ribosomal populations in Escherichia coli cells grown in different media. Mol Gen Genet 119(3): 249-258.

Dey, D., Bochkariov, D.E., Jokhadze, G.G., and Traut, R.R. 1998. Cross-linking of selected residues in the N- and C-terminal domains of Escherichia coli protein L7/L12 to other ribosomal proteins and the effect of elongation factor Tu. J Biol Chem 273(3): 16701676

Dey, D., Oleinikov, A.V., and Traut, R.R. 1995. The hinge region of Escherichia coli ribosomal protein L7/L12 is required for factor binding and GTP hydrolysis. Biochimie 77(12): 925-930.

Diaconu, M., Kothe, U., Schlunzen, F., Fischer, N., Harms, J.M., Tonevitsky, A.G., Stark, H., Rodnina, M.V., and Wahl, M.C. 2005. Structural basis for the function of the ribosomal L7/12 stalk in factor binding and GTPase activation. Cell 121(7): 991-1004.

Dijk, J., Garrett, R.A., and Muller, R. 1979. Studies on the binding of the ribosomal protein complex L7/12-L10 and protein L11 to the 5'-one third of 23S RNA: a functional centre of the 50S subunit. Nucleic Acids Res 6(8): 2717-2729.

Draper, D.E. and Reynaldo, L.P. 1999. RNA binding strategies of ribosomal proteins. Nucleic Acids Res 27(2): 381-388.

Drenth, J. 1994. Principles of protein X-ray crystallography. Springer, New York.

Egebjerg, J., Douthwaite, S.R., Liljas, A., and Garrett, R.A. 1990. Characterization of the binding sites of protein L11 and the L10.(L12)4 pentameric complex in the GTPase domain of 23 S ribosomal RNA from Escherichia coli. J Mol Biol 213(2): 275-288.

Endo, Y., Mitsui, K., Motizuki, M., and Tsurugi, K. 1987. The mechanism of action of ricin and related toxic lectins on eukaryotic ribosomes. The site and the characteristics of the modification in $28 \mathrm{~S}$ ribosomal RNA caused by the toxins. J Biol Chem 262(12): 59085912.

Endo, Y. and Wool, I.G. 1982. The site of action of alpha-sarcin on eukaryotic ribosomes. The sequence at the alpha-sarcin cleavage site in $28 \mathrm{~S}$ ribosomal ribonucleic acid. $J$ Biol Chem 257(15): 9054-9060. 
Fakunding, J.L., Traut, R.R., and Hershey, J.W. 1973. Dependence of initiation factor IF-2 activity on proteins L7 and L12 from Escherichia coli $50 \mathrm{~S}$ ribosomes. $J$ Biol Chem 248(24): 8555-8559.

Fernandez-Puentes, C. and Vazquez, D. 1977. Effects of some proteins that inactivate the eukaryotic ribosome. FEBS Lett 78(1): 143-146.

Frank, J. and Agrawal, R.K. 2000. A ratchet-like inter-subunit reorganization of the ribosome during translocation. Nature 406(6793): 318-322.

Gavrilova, L.P., Kostiashkina, O.E., Koteliansky, V.E., Rutkevitch, N.M., and Spirin, A.S. 1976. Factor-free ("non-enzymic") and factor-dependent systems of translation of polyuridylic acid by Escherichia coli ribosomes. J Mol Biol 101(4): 537-552.

Goldberg, J. 1999. Structural and functional analysis of the ARF1-ARFGAP complex reveals a role for coatomer in GTP hydrolysis. Cell 96(6): 893-902.

Gonzalo, P. and Reboud, J.P. 2003. The puzzling lateral flexible stalk of the ribosome. Biol Cell 95(3-4): 179-193.

Griaznova, O. and Traut, R.R. 2000. Deletion of C-terminal residues of Escherichia coli ribosomal protein L10 causes the loss of binding of one L7/L12 dimer: ribosomes with one L7/L12 dimer are active. Biochemistry 39(14): 4075-4081.

Gualerzi, C.O. and Pon, C.L. 1990. Initiation of mRNA translation in prokaryotes. Biochemistry 29(25): 5881-5889.

Gudkov, A.T. 1997. The L7/L12 ribosomal domain of the ribosome: structural and functional studies. FEBS Lett 407(3): 253-256.

Gudkov, A.T. and Behlke, J. 1978. The N-terminal sequence protein of L7/L 12 is responsible for its dimerization. Eur J Biochem 90(2): 309-312.

Gudkov, A.T., Behlke, J., Vtiurin, N.N., and Lim, V.I. 1977. Tertiary and quaternary structure for ribosomal protein L7 in solution. FEBS Lett 82(1): 125-129.

Gudkov, A.T., Bubunenko, M.G., and Gryaznova, O.I. 1991. Overexpression of L7/L12 protein with mutations in its flexible region. Biochimie 73(11): 1387-1389.

Gudkov, A.T., Gongadze, G.M., Bushuev, V.N., and Okon, M.S. 1982. Proton nuclear magnetic resonance study of the ribosomal protein L7/L12 in situ. FEBS Lett 138(2): 229-232.

Gudkov, A.T., Tumanova, L.G., Gongadze, G.M., and Bushuev, V.N. 1980. Role of different regions of ribosomal proteins L7 and L10 in their complex formation and in the interaction with the ribosomal $50 \mathrm{~S}$ subunit. FEBS Lett 109(1): 34-38.

Gudkov, A.T., Tumanova, L.G., Venyaminov, S.Y., and Khechinashvilli, N.N. 1978. Stoichiometry and properties of the complex between ribosomal proteins L7 and L10 in solution. FEBS Lett 93(2): 215-218.

Gutell, R.R. 1996. Ribosomal RNA: Structure, Evolution, Processing and Function in Protein Biosynthesis. CRC Press, Boca Raton, FL, 1996.

Hamel, E., Koka, M., and Nakamoto, T. 1972. Requirement of an Escherichia coli $50 \mathrm{~S}$ ribosomal protein component for effective interaction of the ribosome with $\mathrm{T}$ and $\mathrm{G}$ factors and with guanosine triphosphate. $J$ Biol Chem 247(3): 805-814.

Hanson, C.L., Fucini, P., Ilag, L.L., Nierhaus, K.H., and Robinson, C.V. 2003. Dissociation of intact Escherichia coli ribosomes in a mass spectrometer. Evidence for conformational change in a ribosome elongation factor G complex. J Biol Chem 278(2): 1259-1267.

Hansson, S., Singh, R., Gudkov, A.T., Liljas, A., and Logan, D.T. 2005. Crystal structure of a mutant elongation factor G trapped with a GTP analogue. FEBS Lett 579(20): 44924497.

Hardy, S.J. 1975. The stoichiometry of the ribosomal proteins of Escherichia coli. Mol Gen Genet 140(3): 253-274. 
Harms, J., Schluenzen, F., Zarivach, R., Bashan, A., Gat, S., Agmon, I., Bartels, H., Franceschi, F., and Yonath, A. 2001. High resolution structure of the large ribosomal subunit from a mesophilic eubacterium. Cell 107(5): 679-688.

Hartz, D., McPheeters, D.S., and Gold, L. 1989. Selection of the initiator tRNA by Escherichia coli initiation factors. Genes Dev 3(12A): 1899-1912.

Hensens, O.D., Albers-Schonberg, G., and Anderson, B.F. 1983. The solution conformation of the peptide antibiotic thiostrepton: a 1H NMR study. $J$ Antibiot (Tokyo) 36(7): 799813.

Hunt, T.W., Fields, T.A., Casey, P.J., and Peralta, E.G. 1996. RGS10 is a selective activator of G alpha i GTPase activity. Nature 383(6596): 175-177.

Ilag, L.L., Videler, H., McKay, A.R., Sobott, F., Fucini, P., Nierhaus, K.H., and Robinson, C.V. 2005. Heptameric (L12)6/L10 rather than canonical pentameric complexes are found by tandem MS of intact ribosomes from thermophilic bacteria. Proc Natl Acad Sci U S A 102(23): 8192-8197.

Inoue-Yokosawa, N., Ishikawa, C., and Kaziro, Y. 1974. The role of guanosine triphosphate in translocation reaction catalyzed by elongation factor G. J Biol Chem 249(13): 43214323.

Kaltschmidt, E. and Wittmann, H.G. 1970. Ribosomal proteins. VII. Two-dimensional polyacrylamide gel electrophoresis for fingerprinting of ribosomal proteins. Anal Biochem 36(2): 401-412.

Karimi, R., Pavlov, M.Y., Buckingham, R.H., and Ehrenberg, M. 1999. Novel roles for classical factors at the interface between translation termination and initiation. $\mathrm{Mol}$ Cell 3(5): 601-609.

Kastner, B., Stoffler-Meilicke, M., and Stoffler, G. 1981. Arrangement of the subunits in the ribosome of Escherichia coli: demonstration by immunoelectron microscopy. Proc Natl Acad Sci U S A 78(11): 6652-6656.

Kawakita, M., Arai, K., and Kaziro, Y. 1974. Interactions between elongation factor tuguanosine triphosphate and ribosomes and the role of ribosome-bound transfer RNA in guanosine triphosphatase reaction. J Biochem (Tokyo) 76(4): 801-809.

Kaziro, Y. 1978. The role of guanosine 5'-triphosphate in polypeptide chain elongation. Biochim Biophys Acta 505(1): 95-127.

Kischa, K., Moller, W., and Stoffler, G. 1971. Reconstitution of a GTPase activity by a 50S ribosomal protein and E. coli. Nat New Biol 233(36): 62-63.

Kisselev, L.L. and Buckingham, R.H. 2000. Translational termination comes of age. Trends Biochem Sci 25(11): 561-566.

Kjeldgaard, M., Nissen, P., Thirup, S., and Nyborg, J. 1993. The crystal structure of elongation factor EF-Tu from Thermus aquaticus in the GTP conformation. Structure 1(1): 35-50.

Klaholz, B.P., Myasnikov, A.G., and Van Heel, M. 2004. Visualization of release factor 3 on the ribosome during termination of protein synthesis. Nature 427(6977): 862-865.

Klein, D.J., Moore, P.B., and Steitz, T.A. 2004. The roles of ribosomal proteins in the structure assembly, and evolution of the large ribosomal subunit. J Mol Biol 340(1): 141-177.

Klein, D.J., Schmeing, T.M., Moore, P.B., and Steitz, T.A. 2001. The kink-turn: a new RNA secondary structure motif. Embo J 20(15): 4214-4221.

Kopke, A.K., Leggatt, P.A., and Matheson, A.T. 1992. Structure function relationships in the ribosomal stalk proteins of archaebacteria. J Biol Chem 267(2): 1382-1390.

Kothe, U., Wieden, H.J., Mohr, D., and Rodnina, M.V. 2004. Interaction of helix D of elongation factor Tu with helices 4 and 5 of protein L7/12 on the ribosome. $J$ Mol Biol 336(5): 1011-1021.

Kristensen, O., Laurberg, M., Liljas, A., and Selmer, M. 2002. Is tRNA binding or tRNA mimicry mandatory for translation factors? Curr Protein Pept Sci 3(1): 133-141. 
La Teana, A., Gualerzi, C.O., and Dahlberg, A.E. 2001. Initiation factor IF 2 binds to the alpha-sarcin loop and helix 89 of Escherichia coli $23 \mathrm{~S}$ ribosomal RNA. Rna 7(8): 1173-1179.

Laemmli, U.K. 1970. Cleavage of structural proteins during the assembly of the head of bacteriophage T4. Nature 227(5259): 680-685.

Lake, J.A. 1976. Ribosome structure determined by electron microscopy of Escherichia coli small subunits, large subunits and monomeric ribosomes. J Mol Biol 105(1): 131-139.

Laskowski, R.A., MacArthur, M.W., Moss, D.S., and Thornton, J.M. 1993. PROCHECK: a program to check the stereochemical quality of protein structures. $J$ Appl Cryst 26: 283-291.

Leffers, H., Egebjerg, J., Andersen, A., Christensen, T., and Garrett, R.A. 1988. Domain VI of Escherichia coli $23 \mathrm{~S}$ ribosomal RNA. Structure, assembly and function. J Mol Biol 204(3): 507-522.

Leijonmarck, M., Eriksson, S., and Liljas, A. 1980. Crystal structure of a ribosomal component at 2.6 A resolution. Nature 286(5775): 824-826.

Liao, D. and Dennis, P.P. 1994. Molecular phylogenies based on ribosomal protein L11, L1, L10, and L12 sequences. J Mol Evol 38(4): 405-419.

Liljas, A. 1982. Structural studies of ribosomes. Prog Biophys Mol Biol 40(3): 161-228.

Liljas, A. 1991. Comparative biochemistry and biophysics of ribosomal proteins. Int Rev Cytol 124: 103-136.

Liljas, A., Erikssson, S., Donner, D., and Kurland, C.G. 1978. Isolation and crystallization of stable domains of the protein L7/L12 from Escherichia coli ribosomes. FEBS Lett 88(2): 300-304.

Liljas, A. and Garber, M. 1995. Ribosomal proteins and elongation factors. Curr Opin Struct Biol 5(6): 721-727.

Liljas, A. and Gudkov, A.T. 1987. The structure and dynamics of ribosomal protein L12. Biochimie 69(10): 1043-1047.

Liljas, A. and Newcomer, M.E. 1981. Purification and crystallization of protein complex from Bacillus stearothermophilus ribosomes. J Mol Biol 153(2): 393-398.

Littlefield, J.W., Keller, E.B., Gross, J., and Zamecnik, P.C. 1955. Studies on cytoplasmic ribonucleoprotein particles from the liver of the rat. J Biol Chem 217(1): 111-123.

Lucas-Lenard, J. and Lipmann, F. 1966. Separation of three microbial amino acid polymerization factors. Proc Natl Acad Sci U S A 55(6): 1562-1566.

Luer, C.A. and Wong, K.P. 1980. Conformational stability of ribosomal protein L7/L12: effects of $\mathrm{pH}$, temperature, and guanidinium chloride. Biochemistry 19(1): 176-183.

Maitra, U., Stringer, E.A., and Chaudhuri, A. 1982. Initiation factors in protein biosynthesis. Annu Rev Biochem 51: 869-900.

Martemyanov, K.A., Liljas, A., and Gudkov, A.T. 2000. Extremely thermostable elongation factor $\mathrm{G}$ from Aquifex aeolicus: cloning, expression, purification, and characterization in a heterologous translation system. Protein Expr Purif 18(3): 257-261.

Martemyanov, K.A., Liljas, A., Yarunin, A.S., and Gudkov, A.T. 2001. Mutations in the Gdomain of elongation factor $\mathrm{G}$ from Thermus thermophilus affect both its interaction with GTP and fusidic acid. J Biol Chem 276(31): 28774-28778.

Masullo, M., Parlato, G., De Vendittis, E., and Bocchini, V. 1989. Effect of propan-2-ol on enzymic and structural properties of elongation factor G. Biochem J 261(3): 725-731.

Mesters, J.R., Potapov, A.P., de Graaf, J.M., and Kraal, B. 1994. Synergism between the GTPase activities of EF-Tu.GTP and EF-G.GTP on empty ribosomes. Elongation factors as stimulators of the ribosomal oscillation between two conformations. $J \mathrm{Mol}$ Biol 242(5): 644-654.

Moazed, D., Robertson, J.M., and Noller, H.F. 1988. Interaction of elongation factors EF-G and EF-Tu with a conserved loop in 23S RNA. Nature 334(6180): 362-364. 
Moens, P.D., Wahl, M.C., and Jameson, D.M. 2005. Oligomeric state and mode of selfassociation of Thermotoga maritima ribosomal stalk protein L12 in solution. Biochemistry 44(9): 3298-3305.

Moller, W., Groene, A., Terhorst, C., and Amons, R. 1972. 50-S ribosomal proteins. Purification and partial characterization of two acidic proteins, A 1 and A 2, isolated from 50-S ribosomes of Escherichia coli. Eur J Biochem 25(1): 5-12.

Moller, W., Schrier, P.I., Maassen, J.A., Zantema, A., Schop, E., Reinalda, H., Cremers, A.F., and Mellema, J.E. 1983. Ribosomal proteins L7/L12 of Escherichia coli. Localization and possible molecular mechanism in translation. J Mol Biol 163(4): 553-573.

Montanaro, L., Sperti, S., Mattioli, A., Testoni, G., and Stirpe, F. 1975. Inhibition by ricin of protein synthesis in vitro. Inhibition of the binding of elongation factor 2 and of adenosine diphosphate-ribosylated elongation factor 2 to ribosomes. Biochem J 146(1): 127-131.

Montesano-Roditis, L., Glitz, D.G., Traut, R.R., and Stewart, P.L. 2001. Cryo-electron microscopic localization of protein L7/L12 within the Escherichia coli $70 \mathrm{~S}$ ribosome by difference mapping and Nanogold labeling. J Biol Chem 276(17): 14117-14123.

Moreno, J.M., Drskjotersen, L., Kristensen, J.E., Mortensen, K.K., and Sperling-Petersen, H.U. 1999. Characterization of the domains of E. coli initiation factor IF2 responsible for recognition of the ribosome. FEBS Lett 455(1-2): 130-134.

Morris, R.J., Perrakis, A., and Lamzin, V.S. 2003. ARP/wARP and automatic interpretation of protein electron density maps. Methods Enzymol 374: 229-244.

Mulder, F.A., Bouakaz, L., Lundell, A., Venkataramana, M., Liljas, A., Akke, M., and Sanyal, S. 2004. Conformation and dynamics of ribosomal stalk protein L12 in solution and on the ribosome. Biochemistry 43(20): 5930-5936.

Munishkin, A. and Wool, I.G. 1997. The ribosome-in-pieces: binding of elongation factor EF$\mathrm{G}$ to oligoribonucleotides that mimic the sarcin/ricin and thiostrepton domains of $23 \mathrm{~S}$ ribosomal RNA. Proc Natl Acad Sci U S A 94(23): 12280-12284.

Myasnikov, A.G., Marzi, S., Simonetti, A., Giuliodori, A.M., Gualerzi, C.O., Yusupova, G., Yusupov, M., and Klaholz, B.P. 2005. Conformational transition of initiation factor 2 from the GTP- to GDP-bound state visualized on the ribosome. Nat Struct Mol Biol 12(12): 1145-1149.

Nierhaus, K.H. and Dohme, F. 1974. Total reconstitution of functionally active 50S ribosomal subunits from Escherichia coli. Proc Natl Acad Sci U S A 71(12): 4713-4717.

Nilsson, J. and Nissen, P. 2005. Elongation factors on the ribosome. Curr Opin Struct Biol 15(3): 349-354.

Nissen, P., Hansen, J., Ban, N., Moore, P.B., and Steitz, T.A. 2000a. The structural basis of ribosome activity in peptide bond synthesis. Science 289(5481): 920-930.

Nissen, P., Kjeldgaard, M., and Nyborg, J. 2000b. Macromolecular mimicry. Embo J 19(4): 489-495.

Nissen, P., Kjeldgaard, M., Thirup, S., Polekhina, G., Reshetnikova, L., Clark, B.F., and Nyborg, J. 1995. Crystal structure of the ternary complex of Phe-tRNAPhe, EF-Tu, and a GTP analog. Science 270(5241): 1464-1472.

Noel, J.P. 1997. Turning off the Ras switch with the flick of a finger. Nat Struct Biol 4(9): 677-680.

Noller, H.F., Hoffarth, V., and Zimniak, L. 1992. Unusual resistance of peptidyl transferase to protein extraction procedures. Science 256(5062): 1416-1419.

Nomura, T., Mochizuki, R., Dabbs, E.R., Shimizu, Y., Ueda, T., Hachimori, A., and Uchiumi, T. 2003. A point mutation in ribosomal protein L7/L12 reduces its ability to form a compact dimer structure and to assemble into the GTPase center. Biochemistry 42(16): 4691-4698. 
Nomura, T., Nakano, K., Maki, Y., Naganuma, T., Nakashima, T., Tanaka, I., Kimura, M., Hachimori, A., and Uchiumi, T. 2006. In vitro reconstitution of the GTPase-associated center of the archaebacterial ribosome: the functional features observed in a hybrid form with Escherichia coli 50S subunits. Biochem J.

Nyborg, J. 1998. Possible evolution of factors involved in protein biosynthesis. Acta Biochim Pol 45(4): 883-894.

Ogle, J.M., Brodersen, D.E., Clemons, W.M., Jr., Tarry, M.J., Carter, A.P., and Ramakrishnan, V. 2001. Recognition of cognate transfer RNA by the 30 S ribosomal subunit. Science 292(5518): 897-902.

Ogle, J.M. and Ramakrishnan, V. 2005. Structural insights into translational fidelity. Annu Rev Biochem 74: 129-177.

Oleinikov, A.V., Perroud, B., Wang, B., and Traut, R.R. 1993. Structural and functional domains of Escherichia coli ribosomal protein $\mathrm{L} 7 / \mathrm{L} 12$. The hinge region is required for activity. J Biol Chem 268(2): 917-922.

Otwinowski, Z. and Minor, W. 1996. Processing of x-ray diffraction data collected in the oscillation mode. Methods enzymol 276: 307-326.

Palade, G.E. 1955. A small particulate component of the cytoplasm. J Biophys Biochem Cytol 1(1): 59-68.

Pape, T., Wintermeyer, W., and Rodnina, M.V. 1998. Complete kinetic mechanism of elongation factor Tu-dependent binding of aminoacyl-tRNA to the A site of the E. coli ribosome. Embo J 17(24): 7490-7497.

Pestka, S. 1970. Thiostrepton: a ribosomal inhibitor of translocation. Biochem Biophys Res Commun 40(3): 667-674.

Pettersson, I. 1979. Studies on the RNA and protein binding sites of the E. coli ribosomal protein L10. Nucleic Acids Res 6(7): 2637-2646.

Pettersson, I., Hardy, S.J., and Liljas, A. 1976. The ribosomal protein L8 is a complex L7/L12 and L10. FEBS Lett 64(1): 135-138.

Pettersson, I. and Kurland, C.G. 1980. Ribosomal protein L7/L12 is required for optimal translation. Proc Natl Acad Sci U S A 77(7): 4007-4010.

Pettersson, I. and Liljas, A. 1979. The stoichiometry and reconstitution of a stable protein complex from Escherichia coli ribosomes. FEBS Lett 98(1): 139-144.

Piepenburg, O., Pape, T., Pleiss, J.A., Wintermeyer, W., Uhlenbeck, O.C., and Rodnina, M.V. 2000. Intact aminoacyl-tRNA is required to trigger GTP hydrolysis by elongation factor Tu on the ribosome. Biochemistry 39(7): 1734-1738.

Pioletti, M., Schlunzen, F., Harms, J., Zarivach, R., Gluhmann, M., Avila, H., Bashan, A., Bartels, H., Auerbach, T., Jacobi, C., Hartsch, T., Yonath, A., and Franceschi, F. 2001. Crystal structures of complexes of the small ribosomal subunit with tetracycline, edeine and IF3. Embo $J$ 20(8): 1829-1839.

Polekhina, G., Thirup, S., Kjeldgaard, M., Nissen, P., Lippmann, C., and Nyborg, J. 1996. Helix unwinding in the effector region of elongation factor EF-Tu-GDP. Structure 4(10): 1141-1151.

Ramakrishnan, V. 2002. Ribosome structure and the mechanism of translation. Cell 108(4): 557-572.

Rawat, U., Gao, H., Zavialov, A., Gursky, R., Ehrenberg, M., and Frank, J. 2006. Interactions of the release factor RF1 with the ribosome as revealed by cryo-EM. $J$ Mol Biol 357(4): 1144-1153.

Rawat, U.B., Zavialov, A.V., Sengupta, J., Valle, M., Grassucci, R.A., Linde, J., Vestergaard, B., Ehrenberg, M., and Frank, J. 2003. A cryo-electron microscopic study of ribosomebound termination factor RF2. Nature 421(6918): 87-90.

Rodes, G. 2000. Crystallography Made Crystal Clear: A Guide for Users of Macromolecular Models. Academic Press, San Diego. 
Rodnina, M.V., Daviter, T., Gromadski, K., and Wintermeyer, W. 2002. Structural dynamics of ribosomal RNA during decoding on the ribosome. Biochimie 84(8): 745-754.

Rodnina, M.V., Pape, T., Fricke, R., Kuhn, L., and Wintermeyer, W. 1996. Initial binding of the elongation factor Tu.GTP.aminoacyl-tRNA complex preceding codon recognition on the ribosome. J Biol Chem 271(2): 646-652.

Rodnina, M.V., Savelsbergh, A., Katunin, V.I., and Wintermeyer, W. 1997. Hydrolysis of GTP by elongation factor G drives tRNA movement on the ribosome. Nature 385(6611): 37-41.

Rodnina, M.V., Savelsbergh, A., Matassova, N.B., Katunin, V.I., Semenkov, Y.P., and Wintermeyer, W. 1999. Thiostrepton inhibits the turnover but not the GTPase of elongation factor G on the ribosome. Proc Natl Acad Sci U S A 96(17): 9586-9590.

Rodnina, M.V. and Wintermeyer, W. 1995. GTP consumption of elongation factor Tu during translation of heteropolymeric mRNAs. Proc Natl Acad Sci U S A 92(6): 1945-1949.

Rosendahl, G. and Douthwaite, S. 1993. Ribosomal proteins L11 and L10.(L12)4 and the antibiotic thiostrepton interact with overlapping regions of the $23 \mathrm{~S}$ rRNA backbone in the ribosomal GTPase centre. J Mol Biol 234(4): 1013-1020.

Sambrook, G., Fritsch, E.F., and Maniatis, T. 1989. Molecular cloning: A laboratory manual, second edition., New York.

Sanchez-Madrid, F., Vidales, F.J., and Ballesta, J.P. 1981. Functional role of acidic ribosomal proteins. Interchangeability of proteins from bacterial and eukaryotic cells. Biochemistry 20(11): 3263-3266.

Sanger, F., Nicklen, S., and Coulson, A.R. 1977. DNA sequencing with chain-terminating inhibitors. Proc Natl Acad Sci U S A 74(12): 5463-5467.

Saraste, M., Sibbald, P.R., and Wittinghofer, A. 1990. The P-loop--a common motif in ATPand GTP-binding proteins. Trends Biochem Sci 15(11): 430-434.

Savelsbergh, A., Katunin, V.I., Mohr, D., Peske, F., Rodnina, M.V., and Wintermeyer, W. 2003. An elongation factor G-induced ribosome rearrangement precedes tRNA-mRNA translocation. Mol Cell 11(6): 1517-1523.

Savelsbergh, A., Mohr, D., Wilden, B., Wintermeyer, W., and Rodnina, M.V. 2000. Stimulation of the GTPase activity of translation elongation factor $\mathrm{G}$ by ribosomal protein L7/12. J Biol Chem 275(2): 890-894.

Schluenzen, F., Tocilj, A., Zarivach, R., Harms, J., Gluehmann, M., Janell, D., Bashan, A., Bartels, H., Agmon, I., Franceschi, F., and Yonath, A. 2000. Structure of functionally activated small ribosomal subunit at 3.3 angstroms resolution. Cell 102(5): 615-623.

Schlunzen, F., Zarivach, R., Harms, J., Bashan, A., Tocilj, A., Albrecht, R., Yonath, A., and Franceschi, F. 2001. Structural basis for the interaction of antibiotics with the peptidyl transferase centre in eubacteria. Nature 413(6858): 814-821.

Schmeing, T.M., Seila, A.C., Hansen, J.L., Freeborn, B., Soukup, J.K., Scaringe, S.A., Strobel, S.A., Moore, P.B., and Steitz, T.A. 2002. A pre-translocational intermediate in protein synthesis observed in crystals of enzymatically active 50S subunits. Nat Struct Biol 9(3): 225-230.

Schmidt, F.J., Thompson, J., Lee, K., Dijk, J., and Cundliffe, E. 1981. The binding site for ribosomal protein L11 within $23 \mathrm{~S}$ ribosomal RNA of Escherichia coli. $J$ Biol Chem 256(23): 12301-12305.

Schneider, T.R. and Sheldrick, G.M. 2002. Substructure solution with SHELXD. Acta Crystallogr D Biol Crystallogr 58(Pt 10 Pt 2): 1772-1779.

Schuwirth, B.S., Borovinskaya, M.A., Hau, C.W., Zhang, W., Vila-Sanjurjo, A., Holton, J.M., and Cate, J.H. 2005. Structures of the bacterial ribosome at 3.5 A resolution. Science 310(5749): 827-834.

Shimmin, L.C., Newton, C.H., Ramirez, C., Yee, J., Downing, W.L., Louie, A., Matheson, A.T., and Dennis, P.P. 1989. Organization of genes encoding the L11, L1, L10, and 
L12 equivalent ribosomal proteins in eubacteria, archaebacteria, and eucaryotes. Can J Microbiol 35(1): 164-170.

Shine, J. and Dalgarno, L. 1974. The 3'-terminal sequence of Escherichia coli 16S ribosomal RNA: complementarity to nonsense triplets and ribosome binding sites. Proc Natl Acad Sci U S A 71(4): 1342-1346.

Sievers, A., Beringer, M., Rodnina, M.V., and Wolfenden, R. 2004. The ribosome as an entropy trap. Proc Natl Acad Sci U S A 101(21): 7897-7901.

Spirin, A.S. 1985. Ribosomal translocation: facts and models. Prog Nucleic Acid Res Mol Biol 32: $75-114$

Sprang, S.R. 1997. G protein mechanisms: insights from structural analysis. Annu Rev Biochem 66: 639-678.

Stark, H., Orlova, E.V., Rinke-Appel, J., Junke, N., Mueller, F., Rodnina, M., Wintermeyer, W., Brimacombe, R., and van Heel, M. 1997a. Arrangement of tRNAs in pre- and posttranslocational ribosomes revealed by electron cryomicroscopy. Cell 88(1): 19-28.

Stark, H., Rodnina, M.V., Rinke-Appel, J., Brimacombe, R., Wintermeyer, W., and van Heel, M. 1997b. Visualization of elongation factor Tu on the Escherichia coli ribosome. Nature 389(6649): 403-406.

Stark, H., Rodnina, M.V., Wieden, H.J., van Heel, M., and Wintermeyer, W. 2000. Large-scale movement of elongation factor $\mathrm{G}$ and extensive conformational change of the ribosome during translocation. Cell 100(3): 301-309.

Stark, H., Rodnina, M.V., Wieden, H.J., Zemlin, F., Wintermeyer, W., and van Heel, M. 2002. Ribosome interactions of aminoacyl-tRNA and elongation factor $\mathrm{Tu}$ in the codonrecognition complex. Nat Struct Biol 9(11): 849-854.

Steitz, T.A. and Moore, P.B. 2003. RNA, the first macromolecular catalyst: the ribosome is a ribozyme. Trends Biochem Sci 28(8): 411-418.

Stoffler-Meilicke, M. and Stoffler, G. 1991. The binding site of ribosomal protein L10 in eubacteria and archaebacteria is conserved: reconstitution of chimeric $50 \mathrm{~S}$ subunits. Biochimie 73(6): 797-804.

Strycharz, W.A., Nomura, M., and Lake, J.A. 1978. Ribosomal proteins L7/L12 localized at a single region of the large subunit by immune electron microscopy. J Mol Biol 126(2): 123-140.

Subramanian, A.R. 1975. Copies of proteins L7 and L12 and heterogeneity of the large subunit of Escherichia coli ribosome. J Mol Biol 95(1): 1-8.

Szewczak, A.A. and Moore, P.B. 1995. The sarcin/ricin loop, a modular RNA. J Mol Biol 247(1): 81-98.

Szick, K., Springer, M., and Bailey-Serres, J. 1998. Evolutionary analyses of the 12-kDa acidic ribosomal P-proteins reveal a distinct protein of higher plant ribosomes. Proc Natl Acad Sci U S A 95(5): 2378-2383.

Tate, W.P., Dognin, M.J., Noah, M., Stoffler-Meilicke, M., and Stoffler, G. 1984. The NH2terminal domain of Escherichia coli ribosomal protein L11. Its three-dimensional location and its role in the binding of release factors 1 and 2. J Biol Chem 259(11): 7317-7324.

Taylor, M.M., Glasgow, J.E., and Storck, R. 1967. Sedimentation coefficients of RNA from $70 \mathrm{~S}$ and 80 S ribosomes. Proc Natl Acad Sci U S A 57(1): 164-169.

Terasaki, M., Suzuki, T., Hanada, T., and Watanabe, K. 2004. Functional compatibility of elongation factors between mammalian mitochondrial and bacterial ribosomes: characterization of GTPase activity and translation elongation by hybrid ribosomes bearing heterologous L7/12 proteins. J Mol Biol 336(2): 331-342.

Terhorst, C., Moller, W., Laursen, R., and Wittmann-Liebold, B. 1973. The primary structure of an acidic protein from 50-S ribosomes of Escherichia coli which is involved in GTP hydrolysis dependent on elongation factors G and T. Eur J Biochem 34(1): 138-152. 
Thompson, J., Cundliffe, E., and Stark, M. 1979. Binding of thiostrepton to a complex of 23-S rRNA with ribosomal protein L11. Eur J Biochem 98(1): 261-265.

Thompson, J.D., Gibson, T.J., Plewniak, F., Jeanmougin, F., and Higgins, D.G. 1997. The CLUSTAL_X windows interface: flexible strategies for multiple sequence alignment aided by quality analysis tools. Nucleic Acids Res 25(24): 4876-4882.

Tocilj, A., Schlunzen, F., Janell, D., Gluhmann, M., Hansen, H.A., Harms, J., Bashan, A., Bartels, H., Agmon, I., Franceschi, F., and Yonath, A. 1999. The small ribosomal subunit from Thermus thermophilus at 4.5 A resolution: pattern fittings and the identification of a functional site. Proc Natl Acad Sci U S A 96(25): 14252-14257.

Tokimatsu, H., Strycharz, W.A., and Dahlberg, A.E. 1981. Gel electrophoretic studies on ribosomal proteins L7/L12 and the Escherichia coli $50 \mathrm{~S}$ subunit. J Mol Biol 152(2): 397-412.

Tomsic, J., Vitali, L.A., Daviter, T., Savelsbergh, A., Spurio, R., Striebeck, P., Wintermeyer, W., Rodnina, M.V., and Gualerzi, C.O. 2000. Late events of translation initiation in bacteria: a kinetic analysis. Embo J 19(9): 2127-2136.

Traub, P. and Nomura, M. 1968. Structure and function of E. coli ribosomes. V. Reconstitution of functionally active $30 \mathrm{~S}$ ribosomal particles from RNA and proteins. Proc Natl Acad Sci U S A 59(3): 777-784.

Uchiumi, T., Honma, S., Nomura, T., Dabbs, E.R., and Hachimori, A. 2002. Translation elongation by a hybrid ribosome in which proteins at the GTPase center of the Escherichia coli ribosome are replaced with rat counterparts. J Biol Chem 277(6): 3857-3862.

Uchiumi, T., Hori, K., Nomura, T., and Hachimori, A. 1999. Replacement of L7/L12.L10 protein complex in Escherichia coli ribosomes with the eukaryotic counterpart changes the specificity of elongation factor binding. J Biol Chem 274(39): 27578-27582.

Urlaub, H., Kruft, V., Bischof, O., Muller, E.C., and Wittmann-Liebold, B. 1995. ProteinrRNA binding features and their structural and functional implications in ribosomes as determined by cross-linking studies. Embo J 14(18): 4578-4588.

Valle, M., Gillet, R., Kaur, S., Henne, A., Ramakrishnan, V., and Frank, J. 2003a. Visualizing tmRNA entry into a stalled ribosome. Science 300(5616): 127-130.

Valle, M., Sengupta, J., Swami, N.K., Grassucci, R.A., Burkhardt, N., Nierhaus, K.H., Agrawal, R.K., and Frank, J. 2002. Cryo-EM reveals an active role for aminoacyltRNA in the accommodation process. Embo J 21(13): 3557-3567.

Valle, M., Zavialov, A., Li, W., Stagg, S.M., Sengupta, J., Nielsen, R.C., Nissen, P., Harvey, S.C., Ehrenberg, M., and Frank, J. 2003b. Incorporation of aminoacyl-tRNA into the ribosome as seen by cryo-electron microscopy. Nat Struct Biol 10(11): 899-906.

Vetter, I.R. and Wittinghofer, A. 1999. Nucleoside triphosphate-binding proteins: different scaffolds to achieve phosphoryl transfer. $Q$ Rev Biophys 32(1): 1-56.

Vetter, I.R. and Wittinghofer, A. 2001. The guanine nucleotide-binding switch in three dimensions. Science 294(5545): 1299-1304.

Vila-Sanjurjo, A., Ridgeway, W.K., Seymaner, V., Zhang, W., Santoso, S., Yu, K., and Cate, J.H. 2003. X-ray crystal structures of the WT and a hyper-accurate ribosome from Escherichia coli. Proc Natl Acad Sci U S A 100(15): 8682-8687.

Wahl, M.C., Bourenkov, G.P., Bartunik, H.D., and Huber, R. 2000a. Flexibility, conformational diversity and two dimerization modes in complexes of ribosomal protein L12. Embo J 19(2): 174-186.

Wahl, M.C., Huber, R., Marinkovic, S., Weyher-Stingl, E., and Ehlert, S. 2000b. Structural investigations of the highly flexible recombinant ribosomal protein L12 from Thermotoga maritima. Biol Chem 381(3): 221-229.

Wahl, M.C. and Moller, W. 2002. Structure and function of the acidic ribosomal stalk proteins. Curr Protein Pept Sci 3(1): 93-106. 
Walleczek, J., Schuler, D., Stoffler-Meilicke, M., Brimacombe, R., and Stoffler, G. 1988. A model for the spatial arrangement of the proteins in the large subunit of the Escherichia coli ribosome. Embo J 7(11): 3571-3576.

Watson, J.D. 1964. The Synthesis of Proteins Upon Ribosomes. Bull Soc Chim Biol (Paris) 46: 1399-1425.

Weiel, J. and Hershey, J.W. 1982. The binding of fluorescein-labeled protein synthesis initiation factor 2 to Escherichia coli $30 \mathrm{~S}$ ribosomal subunits determined by fluorescence polarization. $J$ Biol Chem 257(3): 1215-1220.

Wieden, H.J., Wintermeyer, W., and Rodnina, M.V. 2001. A common structural motif in elongation factor Ts and ribosomal protein L7/12 may be involved in the interaction with elongation factor Tu. J Mol Evol 52(2): 129-136.

Wiggers, R.J., Hadian, H., Traut, R.R., Oleinikov, A.V., and Glitz, D.G. 1997. Localization of two domains of a mutant form of Escherichia coli protein L7/L12 that binds the large ribosomal subunit as a single dimer. Biochimie 79(6): 365-372.

Wilson, D.N., Harms, J.M., Nierhaus, K.H., Schlunzen, F., and Fucini, P. 2005. Speciesspecific antibiotic-ribosome interactions: implications for drug development. Biol Chem 386(12): 1239-1252.

Wilson, D.N. and Nierhaus, K.H. 2005. Ribosomal proteins in the spotlight. Crit Rev Biochem Mol Biol 40(5): 243-267.

Wilson, K.S. and Noller, H.F. 1998. Mapping the position of translational elongation factor EF-G in the ribosome by directed hydroxyl radical probing. Cell 92(1): 131-139.

Wimberly, B.T., Brodersen, D.E., Clemons, W.M., Jr., Morgan-Warren, R.J., Carter, A.P., Vonrhein, C., Hartsch, T., and Ramakrishnan, V. 2000. Structure of the 30S ribosomal subunit. Nature 407(6802): 327-339.

Wimberly, B.T., Guymon, R., McCutcheon, J.P., White, S.W., and Ramakrishnan, V. 1999. A detailed view of a ribosomal active site: the structure of the L11-RNA complex. Cell 97(4): 491-502.

Wittmann, H.G., Mussig, J., Piefke, J., Gewitz, H.S., Rheinberger, H.J., and Yonath, A. 1982. Crystallization of Escherichia coli ribosomes. FEBS Lett 146(1): 217-220.

Wood, P.N. 1991. The use of bonding between tRNAs to implement early peptide synthesis. $J$ Mol Evol 33(5): 464-469.

Wool, I.G. and Stöffler, G. 1974. Ribosomes Cold Spring Harbor Laboratory Press. Cold Spring Harbor, NY.

Wriggers, W., Agrawal, R.K., Drew, D.L., McCammon, A., and Frank, J. 2000. Domain motions of EF-G bound to the 70S ribosome: insights from a hand-shaking between multi-resolution structures. Biophys J 79(3): 1670-1678.

Yonath, A.E., Muessig, J., and Tesche, B. 1980. Crystallization of the large ribosomal subunits from Bacillus stearothermophilus. BIOCHEM INT 1(5): 428-435.

Yusupov, M.M., Yusupova, G.Z., Baucom, A., Lieberman, K., Earnest, T.N., Cate, J.H., and Noller, H.F. 2001. Crystal structure of the ribosome at 5.5 A resolution. Science 292(5518): 883-896.

Yusupova, G.Z., Yusupov, M.M., Cate, J.H., and Noller, H.F. 2001. The path of messenger RNA through the ribosome. Cell 106(2): 233-241.

Zavialov, A.V., Buckingham, R.H., and Ehrenberg, M. 2001. A posttermination ribosomal complex is the guanine nucleotide exchange factor for peptide release factor RF3. Cell 107(1): 115-124.

Zavialov, A.V., Hauryliuk, V.V., and Ehrenberg, M. 2005. Guanine-nucleotide exchange on ribosome-bound elongation factor $\mathrm{G}$ initiates the translocation of tRNAs. $J$ Biol 4(2): 9. 


\section{$\underline{\text { Appendices }}$}

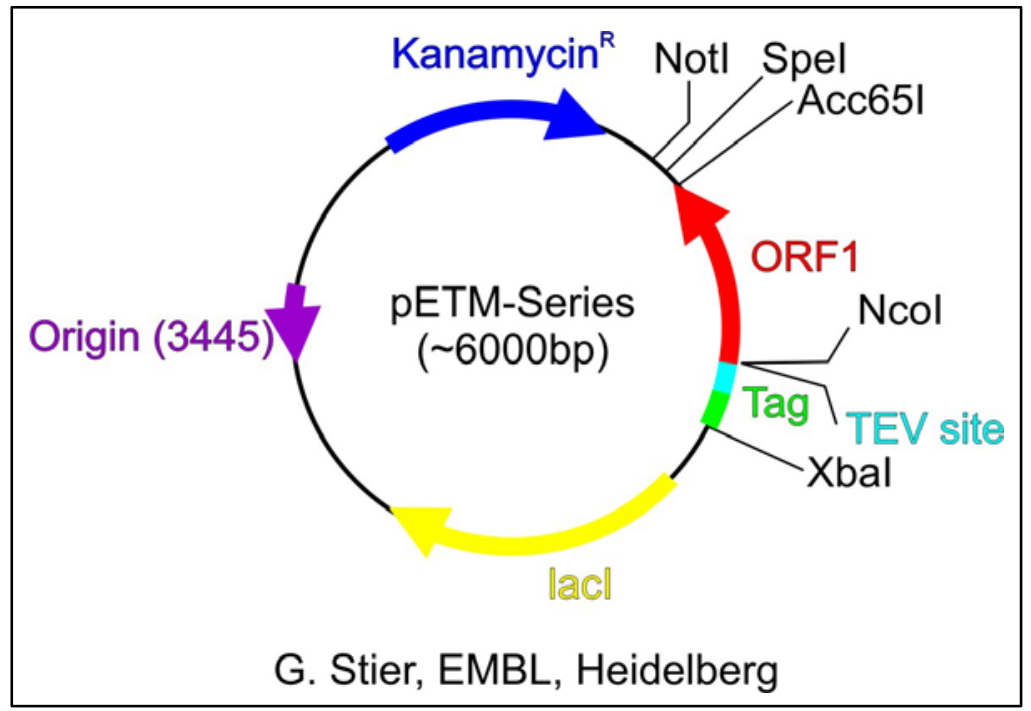

Suppl. Figure 1. The map of the pETM-Series vector.

\begin{tabular}{|lll|}
\hline A & Ala & Alanine \\
R & Arg & Arginine \\
N & Asn & Asparagine \\
D & Asp & Aspartic acid \\
C & Cys & Cysteine \\
Q & Gln & Glutamine \\
E & Glu & Glutamic acid \\
G & Gly & Glycine \\
H & His & Histidine \\
I & Ile & Isoleucine \\
L & Leu & Leucine \\
K & Lys & Lysine \\
M & Met & Methionine \\
F & Phe & Phenylalanine \\
P & Pro & Proline \\
S & Ser & Serine \\
T & Thr & Threonine \\
W & Trp & Tryptophan \\
Y & Tyr & Tyrosine \\
V & Val & Valine \\
\hline
\end{tabular}

Suppl. Figure 2. Amino acids: denominations and abbreviations (1 and 3 letters). 

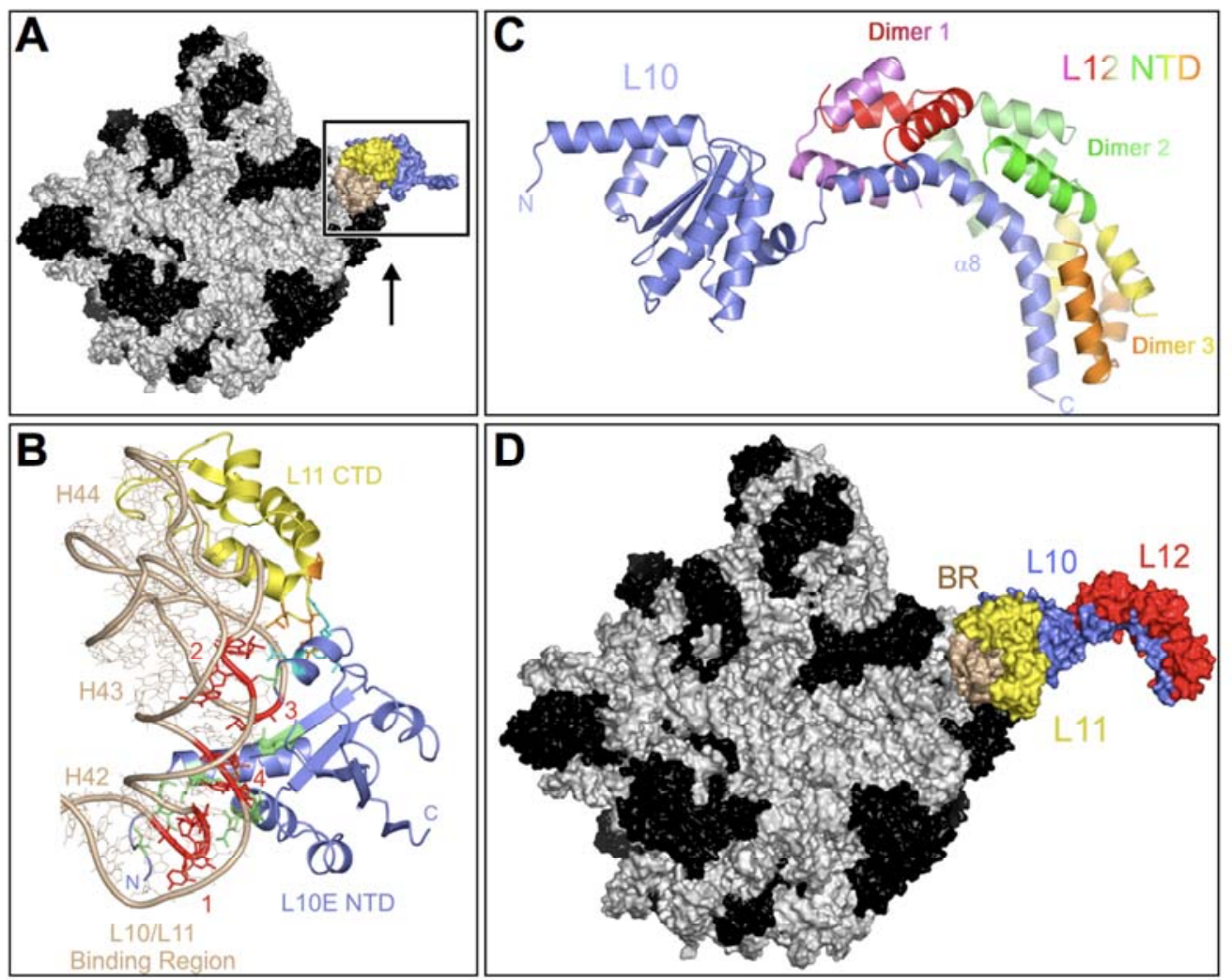

Suppl. Figure 3. (A) Surface view representation of a 50S ribosomal subunit from H. marismortui (PDB accession code 1S72 (Klein et al. 2004)) which was used for the tracing of the L10E NTD. The boxed region represents the L7/L12 stalk, lacking most of its peripheral elements. (B) The structure of the archaeal L10E NTD on the 50S subunit. A detailed view of the L10E NTD bound in the neighborhood of L11 to the L10/L11 binding region of the 23S rRNA is depicted. The L10E NTD (blue) contacts four non-consecutive regions of the rRNA (red). Regions of L10E NTD interacting with the rRNA are in green. A small number of contacts are observed with L11 (cyan and orange residues, respectively). (C) Structure of the tmaL10:(L12 NTD) 6 complex. The C-terminal $\alpha$-helix of L10 accommodates three L12 NTD dimers. (D) Reconstruction of a truncated L7/L12 stalk lacking both hinges and CTDs of L12, by lining up the isolated structure of tmaL10:(L12 NTD) 6 according to the position of L10E NTD on the ribosome.

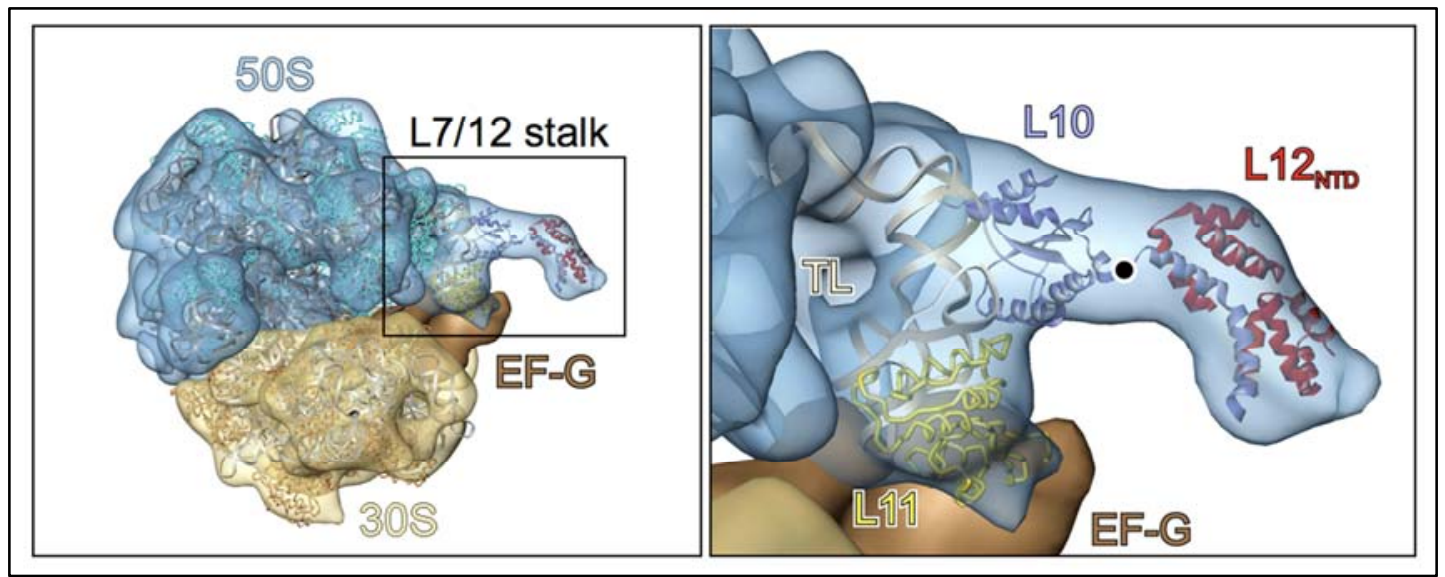

Suppl. Figure 4. (Left) 70S:EF-G:GDP:fusidic acid complex from E. coli, encompassing a truncated L7/L12 stalk that lacked the peripheral elements of L12, namely hinges and CTDs. (Right) The fit of the L10/L11 binding region, L11, and a shortened L10:(L12 NTD) $)_{4}$ crystal structure in the electron density of the 70S:EFG:GDP:fusidic acid complex. 


\section{$\underline{\text { Abbreviations }}$}

\begin{tabular}{|c|c|}
\hline${ }^{\circ} \mathrm{C}$ & degree Celsius \\
\hline $3 \mathrm{D}$ & 3Dimentional \\
\hline$\AA$ & $\operatorname{Angstrom}\left(1 \AA=10^{-10} \mathrm{~m}\right)$ \\
\hline aa & amino acid \\
\hline aae & Aquifex aeolicus \\
\hline ALC & Arc-Like Connection \\
\hline $\begin{array}{l}\text { BLAST } \\
\text { bp }\end{array}$ & $\begin{array}{l}\text { Basic Local Alignment Search Tool } \\
\text { base pair }\end{array}$ \\
\hline $\mathrm{CC}$ & Correlation Coefficient \\
\hline $\mathrm{CCD}$ & Charged Coupled Device \\
\hline $\mathrm{CD}$ & Circular Dichroism \\
\hline $\mathrm{CM}$ & Carboxy Methyl \\
\hline Cryo-EM & Cryo-Electron Microscopy \\
\hline CTD & C-Terminal Domain \\
\hline $\mathrm{ddH} 2 \mathrm{O}$ & double distilled water \\
\hline DEAE & Di-Ethyl Amino Ethyl \\
\hline DESY & Deutsches Elektronen SYnchrotron \\
\hline DMSO & DiMethylSulfOxide \\
\hline DNA & DeoxyriboNucleic Acid \\
\hline DTT & DiThioThreitol \\
\hline eco & Escherichia coli \\
\hline EDTA & Ethylene-Diamine-Tetraacidic Acid \\
\hline $\mathrm{EF}$ & Elongation Factor \\
\hline EF-G & Elongation Factor $\mathrm{G}$ \\
\hline EF-Ts & Elongation Factor Ts \\
\hline $\mathrm{EF}-\mathrm{Tu}$ & Elongation Factor $\mathrm{Tu}$ \\
\hline eIF & eukaryotic Initiation Factor \\
\hline EMBL & European Molecular Biology Laboratory \\
\hline $\mathrm{F}$ & structure Factor \\
\hline fMet & formyl Methionine \\
\hline FOM & Figure Of Merit \\
\hline GAC & GTPase Associated Center \\
\hline GAP & GTPase Activating Protein \\
\hline GAR & GTPase Associated Region \\
\hline GDP & Guanosine DiPhosphate \\
\hline GEF & Guanosine Nucleotide Exchange Factor \\
\hline GMPPNP & guanosine- 5 ' $-[\beta, \gamma$-imido] triphosphate \\
\hline GNBP & Guanosine Nucleotide Binding Protein \\
\hline GST & Glutathione S-Transferase \\
\hline GTP & Guanosine TriPhosphate \\
\hline h & hour \\
\hline
\end{tabular}




\begin{tabular}{|c|c|}
\hline HEPES & N-2-HydroxyEthylPiperazine-N'-2-EthaneSulfonic acid \\
\hline hma & Haloarcula marismortui \\
\hline $\mathrm{I}$ & Intensity \\
\hline IF & Initiation Factor \\
\hline IPTG & IsoPropyl- $\beta$-D-ThioGalactopyranoside \\
\hline ITC & Isothermal Titration Calorimetry \\
\hline $\mathrm{K}$ & Kelvin \\
\hline $\mathrm{kb}$ & kilobase \\
\hline $\mathrm{kD}$ & kilo Dalton \\
\hline 1 & liter \\
\hline LB & Luria Bertani medium \\
\hline $\mathrm{LiCl}$ & Lithium Chloride \\
\hline M & Molarity \\
\hline MAD & Multi-wavelength Anomalous Dispersion \\
\hline MALLS & Multi-Angle Laser Light Scattering \\
\hline $\mathrm{mAU}$ & milli-Absorption Unit \\
\hline MES & 2-(N-Morpholino)-EthaneSulfonic acid \\
\hline $\min$ & minute \\
\hline MIR & Multiple Isomorphous Replacement \\
\hline MPD & 2-methyl 2,4-pentanediol \\
\hline MR & Molecular Replacement \\
\hline mRNA & messenger RNA \\
\hline $\mathrm{NaCl}$ & Natrium Chloride \\
\hline Ni-NTA & Nickel-NitriloTriAcetate \\
\hline $\mathrm{nm}$ & nanometer \\
\hline NMR & Nuclear Magnetic Resonance \\
\hline NTD & N-Terminal Domain \\
\hline OD & Optical Density \\
\hline $\mathrm{P}$ & Phosphate \\
\hline PBS & Phosphate-Buffered Saline \\
\hline PCR & Polymerase Chain Reaction \\
\hline PDB & Protein Data Bank \\
\hline PEG & PolyEthylene Glycol \\
\hline PMSF & phenylmethylsulfonyl fluoride \\
\hline PTC & Peptidyl Transferase Center \\
\hline r.m.s.d. & residual mean-square deviation \\
\hline RF & Release Factor \\
\hline RGS & Regulator of G protein Signaling \\
\hline RMS & Root Mean Square \\
\hline RNA & RiboNucleic Acid \\
\hline rpm & revolutions per minute \\
\hline r-protein & ribosomal protein \\
\hline RRF & Ribosome Recycling Factor \\
\hline RRM & RNA Recognition Motif \\
\hline rRNA & ribosomal RNA \\
\hline
\end{tabular}




\begin{tabular}{ll} 
RT & Room Temperature \\
RU & Resonance Unit \\
S & second \\
S & Svedberg \\
SAD & Single-wavelength Anomalous Diffraction \\
SDS-PAGE & Sodium Dodecyl Sulfate-PolyacrylAmide Gel Electrophoresis \\
SeMet & SelenoMethionine \\
SIR & Single Isomorphous Replacement \\
SIRAS & Single Isomorphous Replacement Anomalous Scattering \\
SLS & Swiss Light Source \\
SPR & Surface Plasmon Resonance \\
SRL & Sarcin-Ricin Loop \\
T C & Temperature in degree Celsius \\
Taq & Thermus aquaticus \\
TEV & Tobacco Etch Virus protease \\
TL & Thiostrepton Loop \\
tma & Thermotoga maritima \\
Tris & Tris-(hydroxymethyl)aminomethane \\
tRNA & transfer RNA \\
tth & Thermus thermophilus \\
U & Unit \\
UV & UltraViolet \\
V & Volume \\
x g & times gravity \\
& \\
\hline
\end{tabular}




\section{$\underline{\text { Acknowledgements }}$}

At the end of my student time, I would like to acknowledge those who contributed for years to my formation and education.

I would like to address my most sincere thanks to my supervisor Priv.-Doz. Dr. Markus Wahl, who helped me enormously during my entire Ph. D. time. I was very fortunate to be part of the group of a brilliant scientist, involved, active and perfectionist, with an opened door for his students' problems at every moment of the day. There are many things to be grateful for, starting with my first protein purification in the middle of the night, encouragements, sparkling ideas, great deal of help with structure solution and interpretation, and initiation of fruitful collaborative projects. I aint't much of a crystallographer, but I surely started to think as one. Danke!

Thank you for excellent collaborations Frank Schlünzen, Jörg Harms, Holger Stark, Niels Fischer, Marina Rodnina, Ute Kothe, Alexander Tonevitski! In addition, I am grateful for the hospitality during my practicum at the University of Witten to Prof. Dr. Marina Rodina and Ute Kothe, as well as to several members of their department.

$I$ am indebted to Prof. Dr. Ralf Ficner for accepting to be the referee of my Ph D thesis. I would also like to thank Prof. Dr. Oliver Einsle for accepting to be the co-referee, as well as to the members of my Ph D committee, namely Prof. Dr. Ahmed Mansouri, Priv.-Doz Dr. Markus Hauck, Prof. Dr. Rüdiger Hardeland and Prof. Dr. Jörg Stülke.

I would like to express my gratitude to Prof. Dr. Reinhard Lührmann for kindly sponsoring a part of my research.

To the head of the Mass Spectrometry Department, Dr. Henning Urlaub, and to the excellent Monika Raabe and Uwe Pleßmann, I thank for the protein identifications, which constituted valuable information for my work. I would like to thank Dr. Ulrich Reidt for a lot of help and advices regarding cloning and protein purification and, in particular, for the help in obtaining the clone for tmaL10. I am grateful to Dr. Gottfried Mieskes and to Prof. Dr. Reinhard Jahn for kindly helping and allowing, respectively, me to perform MALLS experiments in the Department of Neurobiology, to Pawel Burkhardt for taking his time to help me with the ITC measurement, to Dr. Igor Agapov for the help with Biacore measurements, to Dr. Ralf Jauch for the help with CD spectroscopy data collection, and to Dr. Andreas Salvelsbergh for providing tmaEF-G. To Marion Killian and Gordon Dowe, I am thankful for performing the sequencing, to Irene Öchsner for the unlimited supply of the SDS loading buffer and to Thomas Conrad for teaching me how the Äcta works.

I would like to thank the members and former members of our crystallography group, Nina Müllers, Catharina Netter, Elke Penka, Li-chi Chang (who never gets angry...), Thomas Conrad, Marc Drucmann, Vlad Pena, and Ulrich Reidt for generously sharing scientific information. Sunbin Liu, Simon Trowitzsch and Gert Weber, you were the ones who answed a billion questions regarding science and not only. Thank you for all those answers and patience! I wish you all good luck in the future with your experiments! I would like to express my gratitude to all the members of the Department of Cellular Biochemistry for offering their help whenever I needed. Particularly, I thank Heike Beneke for kind advices and guidance in the beginning of my Ph D studies; a special thought to Agnieszka Patkaniowska, Alexandra Andrei and to Berktan Akyildiz (for sharing the same humor). To Iuliane Moses, I am thankful for her kindness, and efficiency in assistantship with all the paper work. 
I would like to acknowledge the Romanian friends here in Göttingen (Alexandra Andrei, Gabriella Ficz, Mara and Marian Pițulescu, Bogdan Papiniu, Vlad and Rodica Pena, Silvio Rizzoli), for all the fruitful discussions and for their support.

I wouldn't be here without a group of wonderful people in the Institute of Human Genetics, Göttingen, that I had the privilege to meet during my Master's studies and from whom I learned a lot. I am indebted to Prof. Dr. med. Wolfgang Engel for the chance to work in his group and for a power-sentence of encouragement, which will linger on forever, and to Prof. Dr. Karim Nayernia for his kindness and a fruitful collaboration in terms of publications. Lab 107, I salute you! Gabriela, Christian, Ewelina, Kerstin, Manyu, Lukasz, Iris, Ilona, Manuela, Jin, Tomek, as well as "my students" Eva and Anne - each of you have a special place in my heart! Ashraf, my best friend here, in Göttingen, I am grateful to you for being here, for advices, sharing, great Indian food and understanding ... I am proud to have worked in the Institute of Cellular Biology and Pathology, Bucharest, founded by George Palade (the discoverer of the ribosome, as a wonderful coincidence related to my present work) and Nicolae Simionescu; I am indebted to Dr. Ina Heltianu for her enormous patience and kindness with a young research assistant and for acknowledging my work, and to Dr. Maya Simionescu and Dr. Monica Raicu for offering me the chance to work in this institute. Many thanks to Adriana Azamfirei! In addition, I would like to thank all my former colleagues, and especially to Irina Fleşeriu, Otilia Postea and Adriana Georgescu, for their advices, kindness, but mostly for our laughters, and the wonderful memories. Mostly, I thank my dear colleague and friend, Cătălina Viallé...I miss our coffee corner! ... But it all started in the Faculty of Biology, Bucharest, where I had the chance to meet great professors and true Ladies who undoubtedly marked my student life and represent who I wish to be like someday: Prof. Dr. Dana Iordachescu, Dr. Cristina Staicu and Dr. Otilia Zărnescu.

I am grateful to all my good friends and former colleagues from home, for showing their care during years: Cătălina Luca, Mihaela Dore, Silvana Constantinescu, Roxana Pietreanu, Irina Matei, Melania Oana and to my best friends Cornelia Mârza and Nadia Butnaru. I would also like to express my appreciation to Gisèle Collombat for her kindness and care.

I am indebted to my family members for all their support and continous encouragement, and especially to my cousins Cristian Pisică, Dafina and Dan Pârvu, Cornelia Trifanov, as well as to my aunt and oncle Dorina and Vasile Pisică. All my love and respect to my grandfather, Gheorghe Pisică; I acknowledge his continous interest in my education.

My very special thanks are addressed to my dear Patrick for being always wonderful, for his unconditional love, care, support and optimism.

Lastly, I would like to thank my dear parents, Elena and Sebastian for being truly the best parents in the world, for their enourmous devotion, love and for making me the happiest (spoiled) child ever.

This work is dedicated to the memory of my beloved aunt Liliana and my grandparents Maria, George and Filofteia (Gica), who left too soon. 


\section{Curriculum Vitae}

Mihaela Ştefania Diaconu, born on August $20^{\text {th }} 1977$ in Bucharest, Romania

\section{Education and professional experience}

1992-1996 “Grigore Moisil” High School, Bucharest, Romania, Baccalaureate

1996-2000～B. Sc. in Biochemistry, University of Bucharest, Romania

Project: Analysis of the biochemical properties of glucose transporters

Advisor: Prof. Dr. Dana Iordachescu

2000-2001 Research assistant, Institute of Cellular Biology and Pathology, Bucharest, Romania

Project: Endothelial nitric oxide synthase gene polymorphism is common to both Fabry disease and atherosclerosis

Advisors: Dr. Constantina Heltianu, Dr. Maya Simionescu

2000-2002 M. Sc. in Biochemistry and Molecular Biology, Institute of Human Genetics, Göttingen, Germany/Faculty of Biology, University of Bucharest, Romania

Project: The PHGPx gene in oligoasthenozoospermia and its expression in murine tissue

Advisors: Prof. Dr. med. Wolfgang Engel, Dr. Karim Nayernia/Prof. Dr.

Dana Iordachescu, Dr. Otilia Zarnescu

2003-2006 Ph. D. studies, Cellular Biochemistry/X-Ray Crystallography Department, Max-Planck Institute for Biophysical Chemistry, Göttingen, Germany

Project: Structural and functional characterization of the L7/L12 ribosomal stalk

Advisor: Priv.-Doz. Dr. Markus C. Wahl

\section{Publications}

- Nayernia K, Diaconu M, Aumuller G, Wennemuth G, Schwandt I, Kleene K, Kühn H, Engel W. Phospholipid hydroperoxide glutathione peroxidase: expression pattern during testicular development in mouse and evolutionary conservation in spermatozoa. Mol Reprod Dev 2004; 67(4): 458-464

- Heltianu C, Costache G, Gafencu A, Diaconu M, Bodeanu M, Cristea C, Azibi $\mathrm{K}$, Poenaru L, Simionescu M. Relationship of eNOS gene variants to diseases that have in common an endothelial cell dysfunction. J Cell Mol Med 2005; 9(1): 135-142

- Diaconu M, Kothe U, Schlünzen F, Fischer N, Harms JM, Tonevitsky AG, Stark $\mathrm{H}$, Rodnina MV, Wahl MC. Structural basis for the function of the ribosomal L7/L12 stalk in factor binding and GTPase activation. Cell 2005; 121(7): 9911004

- Diaconu M, Wahl MC. Filling a gap in the ribosomal GAP. MPIbpc News, 10/2005:1-7

- Diaconu M, Tangat Y, Kühn H, Michelmann HW, Schreiber G, Haidl G, Glander H, Müller C, Engel W, Nayernia K. Failure of phospholipid hydroperoxide glutathione peroxidase expression in oligoasthenozoospermia and mutations in the PHGPx gene (in press) 